\title{
Coronal bright points
}

\section{Maria S. Madjarska ${ }^{1}$}

Received: 9 August 2018 / Accepted: 11 February 2019 / Published online: 15 March 2019 (c) The Author(s) 2019

\begin{abstract}
Coronal bright points (CBPs) are a fundamental class of solar activity. They represent a set of low-corona small-scale loops with enhanced emission in the extreme-ultraviolet and X-ray spectrum that connect magnetic flux concentrations of opposite polarities. CBPs are one of the main building blocks of the solar atmosphere outside active regions uniformly populating the solar atmosphere including active region latitudes and coronal holes. Their plasma properties classify them as downscaled active regions. Most importantly, their simple structure and short lifetimes of less than $20 \mathrm{~h}$ that allow to follow their full lifetime evolution present a unique opportunity to investigate outstanding questions in solar physics including coronal heating. The present Living Review is the first review of this essential class of solar phenomena and aims to give an overview of the current knowledge about the CBP general, plasma and magnetic properties. Several transient dynamic phenomena associated with CBPs are also briefly introduced. The observationally derived energetics and the theoretical modelling that aims at explaining the CBP formation and eruptive behaviour are reviewed.
\end{abstract}

Keywords Sun: coronal loops $\cdot$ Sun: activity $\cdot$ Sun: corona

\section{Contents}

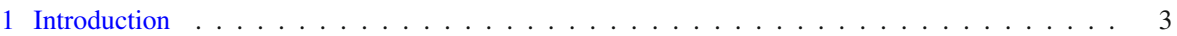

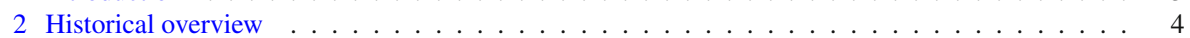

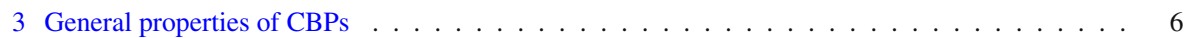

3.1 Morphology ............................ 6

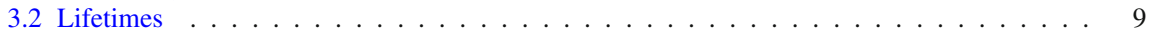

3.3 Solar disk distribution and $\mathrm{N}-\mathrm{S}$ hemisphere asymmetry . . . . . . . . . . . . . . . . . . . . . . . . . . . . . . . 15

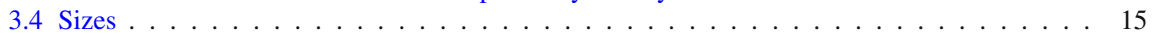

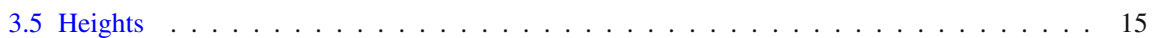

3.6 Occurrence rate . . . . . . . . . . . . . . . . . . . . . . 17

3.7 Solar cycle variation . . . . . . . . . . . . . . . . . . . . . . . . . 18

$凶 \quad$ Maria S. Madjarska

madjarska@mps.mpg.de

1 Max Planck Institute for Solar System Research, Justus-von-Liebig-Weg 3, 37077 Göttingen, Germany 


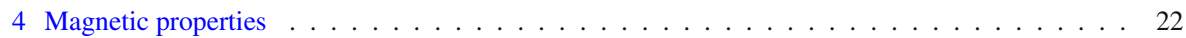

4.1 Photospheric magnetic fields . . . . . . . . . . . . . . . . . . . . . . 22

4.2 Magnetic topology . . . . . . . . . . . . . . . . . . . . . 27

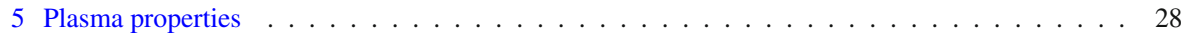

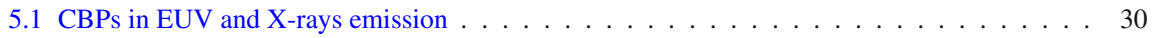

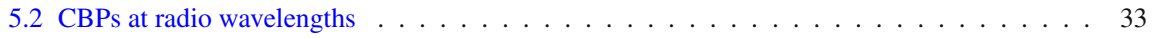

5.3 Doppler and non-thermal velocities . . . . . . . . . . . . . . . . . . . . . . . . . . . . . . . . . . . . .

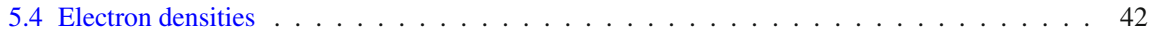

5.5 Temperatures . . . . . . . . . . . . . . . . . . . . . . . . 45

5.6 Plasma filling factor . . . . . . . . . . . . . . . . . . . . . . . . . 48

5.7 Differential emission measure . . . . . . . . . . . . . . . . . . . . . . . . . 49

5.8 Relative element abundances . . . . . . . . . . . . . . . . . . . . . 51

6 Oscillations in CBPs . . . . . . . . . . . . . . . . . . . . . . . 52

7 Transient phenomena associated with CBPs . . . . . . . . . . . . . . . . . . . 54

7.1 Microflaring . . . . . . . . . . . . . . . . . . . . 54

7.2 Eruptions from CBPs: macrospicules, mini-filaments, jets and mini-CMEs . . . . . . . . . 56

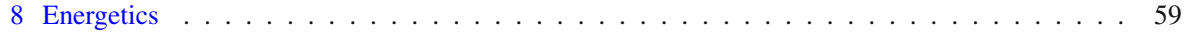

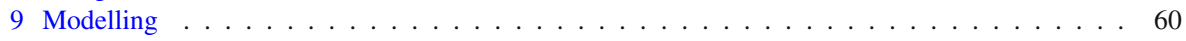

9.1 Ad-hoc heating models . . . . . . . . . . . . . . . . . . . . . 61

9.2 Convergence and cancellation models . . . . . . . . . . . . . . . . . . . . 62

9.3 Separator reconnection models . . . . . . . . . . . . . . . . . . . . . . . 64

9.4 Current-sheet dissipation induced by footpoint motion models . . . . . . . . . . . . 68

10 Conclusions and perspectives . . . . . . . . . . . . . . . . . . . . . . 69

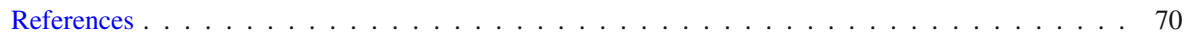

\section{Abbreviations}

CBP Coronal bright point

XBP X-ray bright point

QS Quiet Sun

$\mathrm{CH} \quad$ Coronal hole

AR Active region

CME Coronal mass ejection

EUV Extreme ultraviolet

OSO Orbital Solar Observatory

SMM Solar Maximum Mission

VLA Very Large Array

SOHO Solar Heliospheric Observatory

TRACE Transition Region and Coronal Explorer

SDO Solar Dynamics Observatory

ATM Apollo Telescope Mount

AIA Atmospheric Imaging Assembly

HMI Helioseismic and Magnetic Imager

IRIS Interface Region Imaging Spectrograph

SXT Soft X-ray Telescope

STEREO Solar Terrestrial Relations Observatory

SECCHI Sun Earth Connection Coronal and Heliospheric Investigation

BBSO Big Bear Solar Observatory

EUVI Extreme Ultraviolet Imager

SJ Slit jaw 


\section{Introduction}

The solar corona seen in extreme-ultraviolet (EUV) and X-rays is mainly composed of loops with a wide spectrum of sizes. Luminous active region (AR) loops together with AR interconnecting also bright loops overlay the strong magnetic fields of sunspots and faculae $\left(1 \times 10^{20} \mathrm{Mx}\right.$ to $3 \times 10^{22} \mathrm{Mx}$, half of total unsigned flux, van Driel-Gesztelyi and Green 2015, and the references therein) dominating the solar corona during the solar maximum at latitudes of up to $\pm 35^{\circ}$. Outside these regions small magnetic flux concentrations of up to $1 \times 10^{20} \mathrm{Mx}$ with a salt and pepper pattern are omnipresent and involved in various processes including emergence, coalescence, fragmentation, and cancellation. Unipolar regions also exist in the quiet Sun producing large (usually fainter) sometimes trans-equatorial loops that create hazy background emission in the EUV. Some of the small-scale magnetic structures confine plasma that is heated to up to million degrees producing the so-called coronal bright points (CBPs) in the quiet Sun, coronal holes and in the vicinity of active regions. Playing a certain role in the heating of the solar corona, CBPs were a subject of relatively intensive research since their discovery in 1969. At solar minimum the solar corona, sometimes with no active regions present, is mostly populated by CBPs and fainter larger loops (see Fig. 1) as well as occasional coronal holes, while remaining heated to million degrees. Over the past few decades both observational and theoretical state-of-the-art studies are building up knowledge that aims to uncover the physical mechanisms that operate, possibly through a co-action, in the solar atmosphere creating this unique physical phenomenon.

A dedicated review on coronal loops by Reale (2014) gives a comprehensive insight on the present state of knowledge on these coronal features also referring to some of the properties of CBPs that occupy the low end of the size spectrum of coronal loops. The

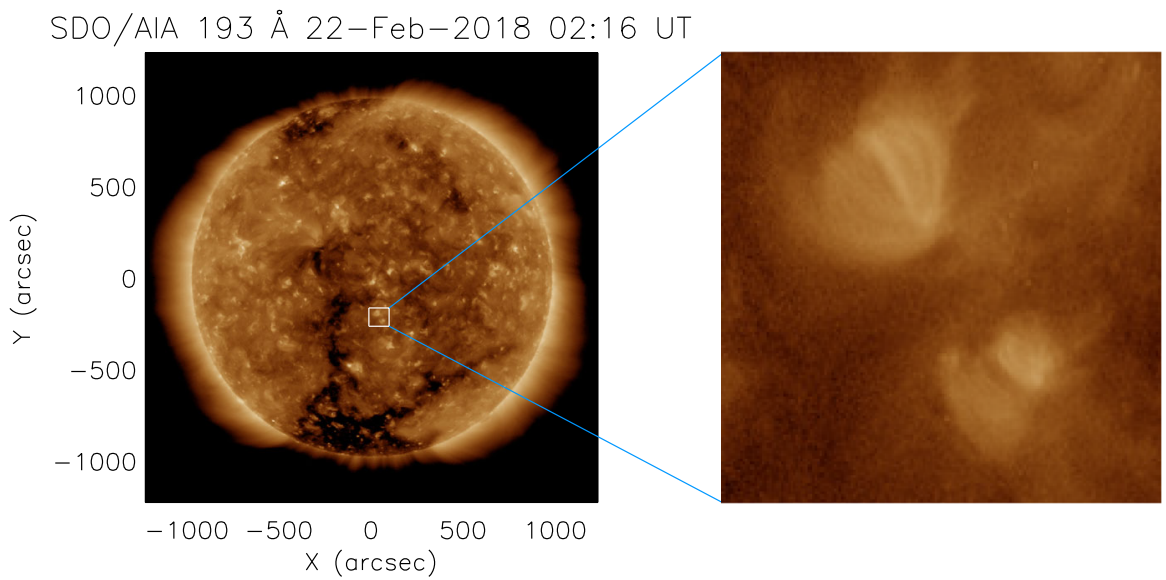

Fig. 1 Left: full disk SDO/AIA image in the Fe XII $193 \AA$ channel during the minimum of solar cycle 24 entirely dominated by the quiet Sun and coronal holes. A white square outlines a field-of-view that contains two bright points. Right: enlarged field-of-view of the outlined region in the left panel image (size of $\left.110^{\prime \prime} \times 100^{\prime \prime}\right)$ 
present review focuses exclusively on the subject of CBPs and the dynamic phenomena that occur during their evolution. A historical overview first proceeds through the scientific contribution of the space missions and ground-based observatories (Sect. 2). Next the review reports on the findings on the general physical properties of CBPs that define them as a unique class of solar activity phenomenon (Sect. 3). The magnetic properties including associated photospheric flux formation and evolution, as well as coronal magnetic structures derived by applying various potential field models are presented in Sect. 4. This is followed by an outline on the CBP plasma properties that give important clues on the physical mechanisms at work but also constrain the general theoretical model definition (Sect. 5). Oscillations detected in CBPs and their interpretation are addressed in Sect. 6. The dynamic activity of CBPs that produces a unique class of small-scale dynamic transients are given in the follow-up Sect. 7. Section 8 is dedicated to the energetics of CBPs obtained from observations. The final section of this review delivers an overview on the theoretical modelling of CBPs (Sect. 9). All sections introduce the knowledge on each subject by progressing in time through the build up of the understanding of a particular property, a physical parameter or a related feature. Finally, Sect. 10 gives the main conclusions and perspectives on the future research opportunities and requirements to be able to advance on our understanding of the phenomenon coronal bright point.

\section{Historical overview}

The first full-disk X-ray observations of the solar corona with grazing incidence Soft X-ray Telescopes took place during a series of rockets flights (1968-1973) and later by the X-ray spectrographic telescope (S-054, 3-60 A wavelength coverage) of the Apollo Telescope Mount (ATM) on board the Skylab Earth orbiting laboratory launched on 1973 May 14 (Vaiana et al. 1973a,b). These observations revealed that the "quiet homogeneous" solar corona, as it has been known until then, is highly structured with numerous bright point-like X-ray emission sources randomly distributed on the solar disk including polar regions. Thus, the term X-ray bright point (XBP) was introduced. The XBPs were first studied in detail in photographs taken during the 1969 April 8 rocket flight as this experiment was specifically designed to study quiet Sun features. Vaiana et al. (1973a) described the phenomenon as a "small point-like feature of relatively bright soft X-ray emission" that is related to bright spots in the Ca II K network. Occasionally a bright patch was also co-observed in $\mathrm{H} \alpha$ images. Each bright point was associated with bipolar magnetic fragments in predominantly unipolar magnetic regions (Krieger et al. 1971). Vaiana et al. (1973b) concluded that these phenomena represent "the X-ray manifestation of low lying closed bipolar magnetic structures outside active regions". XBPs were found to have short lifetimes of less than a day based on a study of small newly emerging bipolar magnetic features named ephemeral active regions. They appeared to be uniformly distributed across the entire solar disk which indicated that they have a different production mechanism from that of active regions (Vaiana et al. 1973b).

Skylab/ATM (1973-1979) (Vaiana et al. 1976, and see https://history.nasa.gov/SP402/contents.htm) provided the data from which the first fundamental information on 
coronal bright points was obtained including the emission variations at chromospheric, transition-region and coronal temperatures, structure, lifetimes, sizes, occurrence rate, solar disk distribution and solar cycle variations. These data combined with magnetic field information from ground-based observatories like the Big Bear Solar Observatory (BBSO) and Kitt Peak National Observatory established the magnetic flux range of XBPs.

After the Very Large Array (VLA, Thompson et al. 1980, and see http://www. vla.nrao.edu/) became operational in the 1980s, studies have concentrated on the microwave signatures of XBPs as well as radio type III bursts that indicate the presence of energetic particles due to a high energy release. The studies provided evidence on the dynamics and energetics of coronal bright points as well as the only evaluation of their magnetic field at transition-region heights.

The Yohkoh Soft X-ray Telescope (SXT, Tsuneta et al. 1991) hugely enriched the knowledge about the dynamics of XBPs thanks to the higher cadence and continuous monitoring of the solar disk in X-rays. Ejections from XBPs known as X-ray jets were reported and studied for the first time. It also provided a better understanding on the solar cycle variations as well as solar disk distribution of XBPs.

The launch of the Solar Heliospheric Observatory (SOHO, Domingo et al. 1995) established a new era of unprecedented UV and EUV observations of the solar atmosphere. Subsequently XBPs were referred to as EUV BPs as being explored in imaging data from the Extreme-ultraviolet Imaging Telescope (EIT, Delaboudinière et al. 1995, mission completed in July 2010), in longitudinal magnetograms from the Michelson Doppler Imager (MDI, Scherrer et al. 2012, mission completed in April 2011) and in spectroscopic data covering spectral lines with a wide range of formation temperatures from $10^{4}$ to $10^{7} \mathrm{~K}$, obtained by the Coronal Diagnostics Spectrometer (CDS, Harrison et al. 1995, until September 2014) and the Solar Measurements of Emitted Radiation spectrometer (SUMER, Wilhelm et al. 1997; Lemaire et al. 1997, mission ended April 2017). The plasma properties and oscillations as well as the occurrence rate, solar disk distribution and cycle variations were derived. The magnetic properties and heights were obtained, and the dynamic activity including jet and microflaring studies were made.

Thanks to its high spatial resolution, the Transition Region and Coronal Explorer (TRACE, Handy et al. 1999) revealed the fine structure of EUV BPs. All three instruments on board the Hinode mission (Kosugi et al. 2007), the Extreme-ultraviolet Imaging Spectrometer (EIS, Culhane et al. 2007a), the Solar Optical Telescope (SOT, Tsuneta et al. 2008), and the X-ray telescope (XRT, Golub et al. 2007), brought a wealth of knowledge on the bright points' dynamics and associated activity in EUV and X-rays. The Hinode/EIS and XRT instruments contributed with an unprecedented plasma diagnostics including differential emission measure and filling factor of bright points. The electron density and temperature of bright points, and their spatial distribution and temporal variations were also obtained. Studies on oscillations and magnetic topology were made, and unique vector magnetic field data of bright points were taken. The Extreme Ultraviolet Normal Incidence Spectrograph (EUNIS) sounding rocket instrument has left its imprint in the study of bright points with spectroscopic data that provided their Doppler and non-thermal velocities, as well as differential emission measure. Based on EUNIS data the first and only study on relative elemental abun- 
dances of bright points were obtained. The Solar Terrestrial Relations Observatory (STEREO, Kaiser et al. 2008; Driesman et al. 2008) allowed a unique approach for obtaining heights of bright points' loops thanks to the twin spacecrafts (one ahead of Earth in its orbit and the other following behind) stereoscopic measurements with high cadence EUV imaging data. The Solar Dynamics Observatory (SDO, Pesnell et al. 2012) imaging from the Atmospheric Imaging Assembly (AIA, Lemen et al. 2012) and magnetic field data taken by the Helioseismic Magnetic Imager (HMI, Scherrer et al. 2012) had a huge impact on studying the temporal evolution and dynamic activity of bright points including mini coronal mass ejections (mini-CMEs). The Interface Region Imaging Spectrograph (IRIS, De Pontieu et al. 2014) studies report on the transition region Doppler shifts in bright points' loops at unprecedented spectral and spatial resolution.

After almost five decades of intensive studies with ever improving solar instrumentation, the term coronal bright point (CBP) has been adopted to describe a small-scale multi-loop system in the low solar corona that appears in extreme-ultraviolet and X-ray images with enhanced emission (weaker than those of active regions) whilst associated with magnetic bipolar features.

\section{General properties of CBPs}

\subsection{Morphology}

CBPs were initially seen in X-rays (or XBPs) as point-like structures with a diameter of $20^{\prime \prime}-30^{\prime \prime}$ and a bright core of $5^{\prime \prime}-10^{\prime \prime}$ that is surrounded by diffused emission (e.g., Vaiana et al. 1973b; Golub et al. 1974) (Fig. 2). XBPs were associated with small regions of opposite polarity magnetic flux (Krieger et al. 1971) which doubtlessly suggested that these phenomena are "the X-ray manifestation of low lying closed bipolar magnetic structures outside active regions" (Vaiana et al. 1973b). Later, the high-

Fig. 2 Full disk X-ray image taken with the S-054 spectroheliographic telescope with some of the XBPs encircled. Image reproduced with permission from Nolte et al. (1979), copyright by Springer

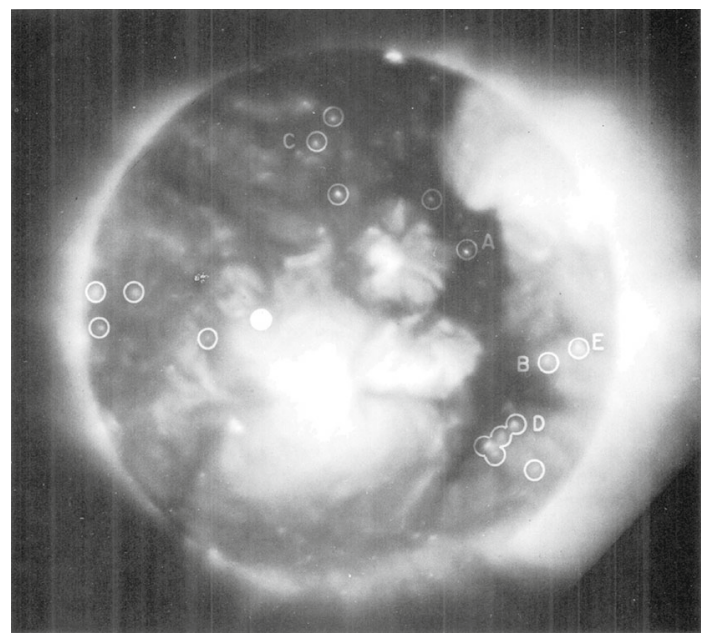


$\Delta t \sim 1.5 \cdot 3.0 \mathrm{hrs}$.

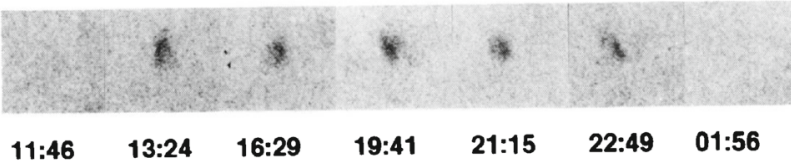

$\Delta t=6 \min$

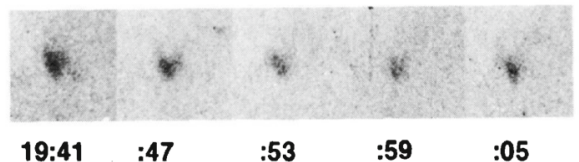

$30.000 \mathrm{~km}$

19:41

\section{7}

$: 53$

.59

$: 05$

$$
\Delta t=2 \min
$$

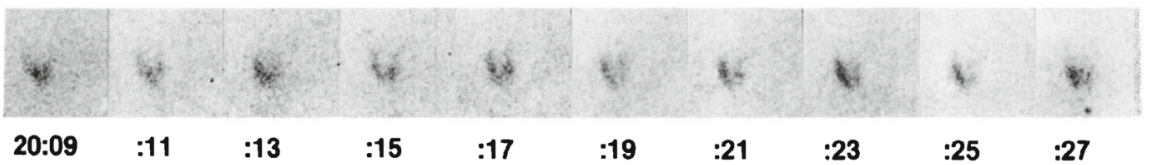

Fig. 3 CBPs in a sequence of Fe XV $284 \AA$ spectroheliograms (negative) taken on 1974 January 19-20. The evolution of a CBP at three different cadences, 1.5-3.0 h, $6 \mathrm{~min}$, and $2 \mathrm{~min}$, is given in the top, middle and bottom panels, respectively. Image reproduced with permission from Sheeley and Golub (1979), copyright by Springer

resolution spectroheliograms (a photograph of the Sun taken at a single wavelength, i.e., a monochromatic image) from the spectroheliograph on Skylab/ATM revealed that the bright 'points' consist of rapidly and independently evolving 2-3 small-scale loops (Fig. 3) with a diameter of $\sim 2500 \mathrm{~km}$ and $\sim 12,500 \mathrm{~km}$ length (Sheeley and Golub 1979). The observations were obtained in the Fe XV $284 \AA[\log T(\mathrm{~K}) \sim 6.3]$ line at a $2^{\prime \prime}$ spatial resolution, unprecedented for its time.

Golub et al. (1974) characterized the typical evolution of CBPs in X-rays. From the analysis of 100 XBPs, they established that the diffused emission always precedes the bright core that vanishes before the final disappearance of the XBPs. The core was often found to have almost elliptical shape. The intensity evolution in X-rays revealed that CBPs have a rapid growth and a slow decay. The average growth rate was estimated at $5^{\prime \prime}$ per hour or $1 \mathrm{~km} \mathrm{~s}^{-1}$ that is similar to the horizontal velocity of supergranulation cells (Golub et al. 1974) indicating a strong relation to the supergranular evolution or/and supergranular flows.

Habbal et al. (1990) investigated the observational properties including the morphological structure and temporal behaviour of quiet Sun and coronal hole CBPs from the emission in six spectral lines formed at chromospheric, transition region, and coronal temperatures using data from the Harvard EUV experiment on Skylab. The data consisted of scans with a $5^{\prime} \times 5^{\prime}$ field-of-view in the Sun's disk centre taken at $5.5 \mathrm{~min}$ cadence and a spatial resolution of $5^{\prime \prime}$. CBPs in the two topologically different regions were found to be located at the network boundaries and cover the same range of sizes from $10^{\prime \prime}$ to $40^{\prime \prime}$ (diameter). CBPs also showed no difference related to the properties of the observed region suggesting that their formation and existence is independent 


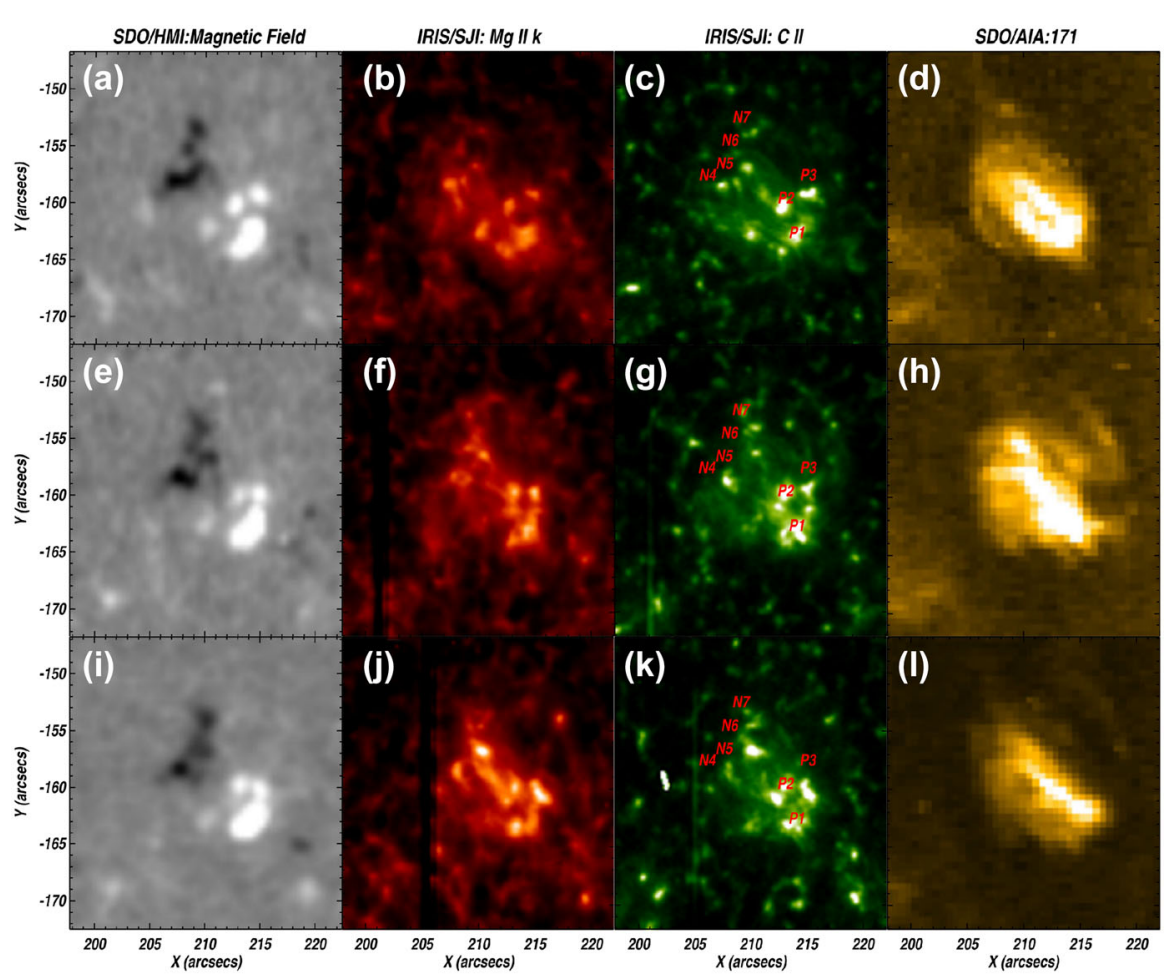

Fig. 4 Temporal evolution of a CBP. From left to right: SDO/HMI longitudinal magnetograms showing the bipole associated with the CBP. IRIS SJ images in the Mg II k $2796 \AA$ passband, SJ images in the C II $1330 \AA$ passband, and SDO/AIA $171 \AA$ images. The top row images are taken at 11:33:51 UT, the middle row at 11:43:19 UT, and the bottom row at 12:03:18 UT. Image reproduced with permission from Kayshap and Dwivedi (2017), copyright by Springer

of the overlying corona, i.e., the specific magnetic topology of the observed region. The emission peaks in six different spectral lines measured simultaneously were not co-spatial, which made the authors conclude that CBPs are composed of a complex of loops at different temperatures.

Due to resolution limitations CBPs were also seen in SOHO/EIT images as a diffused cloud with a bright core or a singular loop (e.g., Longcope et al. 2001; Madjarska et al. 2003; Brown et al. 2001; Pérez-Suárez et al. 2008). Individual small-scale loops were distinguishable in the TRACE Fe IX/X $171 \AA$ passband (hereafter TRACE $171 \AA$ ) (e.g., Ugarte-Urra et al. 2004a, see their Fig. 1) and later Hinode/EIS raster data (1" slit) (e.g., Dere 2008, see their Fig. 2). Recently, Kayshap and Dwivedi (2017) analysed two CBPs in data taken by SDO/AIA and HMI, and IRIS. The CBPs appeared to be composed of dynamically evolving small-scale loops seen in IRIS slit-jaw images (SJI) taken in the Mg II k $2796 \AA$ (chromosphere) and C II $1330 \AA$ (low transition region) passbands (note that mostly the lower part of the loops referred to as loop legs are seen in these passbands), as well as in the SDO/AIA Fe IX/X $171 \AA$ (hereafter AIA $171 \AA$ ) passband that is dominated by emission from plasma at $0.8 \mathrm{MK}$ (Fig. 4). Small-scale low-lying loops that connect the fragments of the opposite polarity mag- 
netic flux are distinguishable in the lower temperature channels, while larger possibly overlying loops are visible in the higher temperature channel although no comparative loop measurements exist. The study on the morphology and evolution only cover the time period of the IRIS observations, i.e., $3.5 \mathrm{~h}$. Mou et al. (2018) followed the full lifetime evolution of CBPs. The CBPs forming from flux emergence initially appear (in AIA $195 \AA$ ) as loops as small as $5^{\prime \prime}$ (diameter of the disk projected feature) that later grow to reach a maximum size of up to $\sim 60^{\prime \prime}$, followed later by a decrease to a size of less than $5^{\prime \prime}$, before fully vanishing. Small-scale dynamically evolving individual loops are seen during the whole lifetime of the CBPs.

To conclude, CBPs are composed of small-scale loops with hotter loops overlaying cooler connecting opposite magnetic flux concentrations found in the network at the junctions of supergranulation cells. CBP loops evolve rapidly at time scales as short as 5 min. Recent high cadence observations, e.g., SDO/AIA and or IRIS, are not yet explored to investigate the evolution of individual CBP loops.

\subsection{Lifetimes}

From the analysis of 100 CBPs identified in X-ray images taken by the Skylab S054 X-ray telescope, Golub et al. (1974) first determined that XBPs have a statistical distribution of lifetimes in X-rays with a mean value of $8 \mathrm{~h}$. The study found a larger number of XBPs with shorter lifetimes and a smaller number of XBPs with longer lifetimes. It was established that the life span of XBPs is approximately proportional to their maximum size (not confirmed yet in present data) that can be expressed as

$$
A_{\max }=2.5 \times 10^{7} \tau
$$

where $A$ is measured in $\mathrm{km}$ and $\tau$ in $\mathrm{h}$ (Golub et al. 1974). The typical maximum area reached by XBPs was estimated at $2.0 \pm 1.0 \times 10^{8} \mathrm{~km}^{2}$ (diameter of $20^{\prime \prime} \pm 5^{\prime \prime}$ ). The lifetime spectrum of XBPs was found to be strongly weighted towards shorter lifetimes with the number of XBPs living for more than $48 \mathrm{~h}$ more than 10 times smaller than XBPs with lifetimes from 2 to $48 \mathrm{~h}$ (Golub et al. 1976a, 304 XBPs were analysed). $\mathrm{XBP}$ lifetimes in the two lifetime ranges was best fitted with a four-parameter function

$$
N(t)=N_{s} e^{-t / \tau_{s}}+N_{l} e^{-t / \tau_{l}}
$$

with an average lifetime for the shorter living CBPs of $\tau_{s}=8.7 \pm 0.2 \mathrm{~h}$ and an average lifetime for the long-living XBPs of $\tau_{l}=35 \pm 4 \mathrm{~h}$. The number of XBPs with shorter lifetimes is denoted as $N_{s}$ and $N_{l}$ with longer, with a relation of $N_{s} \sim 10 N_{l}$. Long-living XBPs were found to be concentrated in the latitude belt of $\pm 30^{\circ}$ from the equator, i.e., in active region latitudes, while XBPs with lifetimes of less than 2 days appear over a larger range of latitudes. CBPs show similar lifetimes to those of supergranulation cells which strongly hints towards their close relationship. After the launch of the Yohkoh satellite, the subject of XBPs' lifetimes was revisited by Harvey et al. (1993) using full-disk Yohkoh/SXT observations. In total 518 XBPs were studied, from which $34 \%$ were located in coronal holes $(\mathrm{CHs}), 60 \%$ in the quiet 

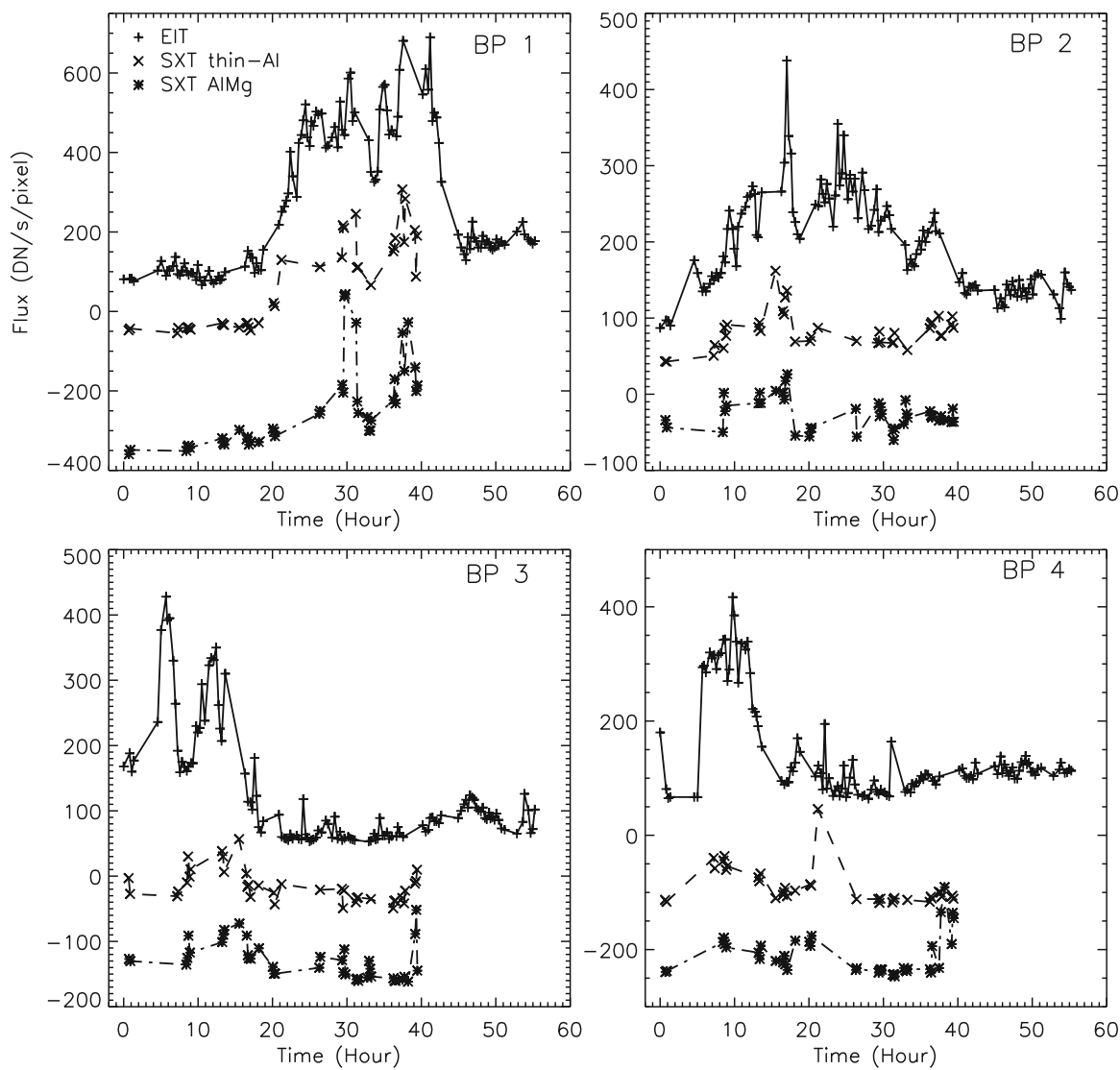

Fig. 5 Lightcurves of four CBPs identified in SOHO/EIT $195 \AA$ (denoted by plus symbols, '十'), Yohkoh/SXT thin_Al filter $(\times)$, and AlMg $(*)$ images. Image reproduced with permission from Zhang et al. (2001), copyright by Springer

Sun (QS) and 6\% in ARs. Their lifetimes were estimated to an average of $12.6 \mathrm{~h}$, $13 \mathrm{~h}$ and $11.7 \mathrm{~h}$, respectively, indicating that no significant difference exists between XBPs formed in regions with different magnetic topology supporting the conclusion of Habbal et al. (1990) on the properties of CBPs in CHs and QS.

The lifetimes of CBPs was studied for the first time in EUV in images taken with SOHO/EIT. Zhang et al. (2001) analysed $55 \mathrm{~h}$ of images taken in the Fe XII $195 \AA$ passband (hereafter SOHO/EIT $195 \AA$ ). . A field-of-view of $780^{\prime \prime} \times 780^{\prime \prime}$ was selected from full-disk images centred at the equator that was tracked during the observing period. The CBP identification was based on a $3 \sigma$ threshold where $\sigma$ is the rms deviation of the intensity flux of the selected quiet Sun region. Yohkoh/SXT images taken with the thin-Al and AlMg filters sensitive to plasma at $\sim 1.5 \mathrm{MK}$ during the same period of time but not as regular as SOHO/EIT were also used. In the observed period the full lifetimes of $48 \mathrm{CBPs}$ were tracked. The average lifetime of CBPs in the SOHO/EIT $195 \AA$ channel was estimated at $20 \mathrm{~h}$. Shorter lifetimes and lower number 

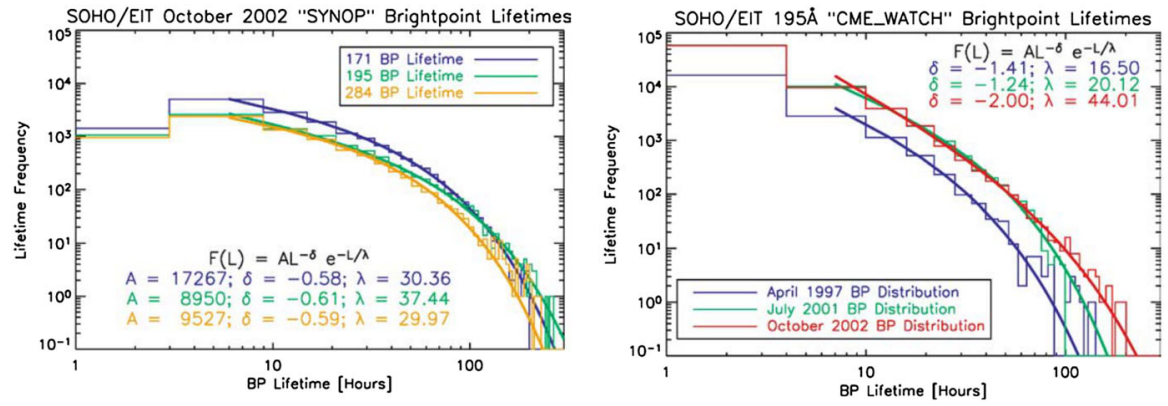

Fig. 6 Lifetime distributions of CBPs from three samples of data taken at a $6 \mathrm{~h}$ cadence (left panel) and from the CME-watch mode at $\sim 12$ min cadence (right panel) in the EIT $195 \AA$ channel. Image reproduced with permission from McIntosh and Gurman (2005), copyright by Springer

of CBPs were found in the Yohkoh/SXT images. Figure 5 shows the lightcurves of four CBPs in simultaneous EUV and X-ray observations. The CBPs become visible in X-rays only for a very short period of time and often during flaring activity, which explains their generally shorter lifetimes in X-rays.

CBP automatic identification and tracking algorithms were also used to obtain the statistical properties of CBPs, including their lifetimes. McIntosh and Gurman (2005) employed a slightly modified CBP identification algorithm developed by Hara and Nakakubo-Morimoto (2003) (for details on the method see Sect. 3.7) that is based on an intensity background threshold. In total $5 \times 10^{6} \mathrm{CBPs}$ were identified and their appearance at different temperatures was investigated. SOHO/EIT had two main data recording modes, a synoptic and a so-called CME-watch. The synoptic mode images were taken every $6 \mathrm{~h}$ in all four channels, namely He II $304 \AA$, Fe IX/X $171 \AA$, Fe XII $195 \AA$ and FeXV $284 \AA$. The CME-watch mode collected data only in the $195 \AA$ passband every $12-17 \mathrm{~min}$. These higher cadence data were employed to study the lifetimes of CBPs. Data obtained from January 1997 until December 2004 were analysed. From the identified CBPs $97 \%$ were located within $600^{\prime \prime}$ of the disk centre. Based on the $6 \mathrm{~h}$ cadence data, it was found that the frequency distribution of CBP lifetimes is a power law at short to intermediate lifetimes and shows exponential behaviour at longer lifetimes (see Fig. 6, left panel). The 12-min cadence data that permit a better coverage of shorter-living CBPs provided a sample of 200,000 CBPs. The power-law indices of these CBP lifetime distributions increase significantly thanks to the better coverage of CBPs with shorter lifetimes which however does not affect the 'turning point' of the distributions (see Fig. 6, right panel). This turning point is assumed as the average lifetime of CBPs $(\sim 10 \mathrm{~h})$. The distribution pattern could also be affected by the underestimation of the CBP duration if the CBPs first appear close to the west limb. The temperature response of the channel used for the identification also plays a significant role with more CBPs and with longer lifetimes in the channels with lower temperature response (see e.g., Zhang et al. 2001).

The lifetime frequency distribution of CBPs were also obtained by Alipour and Safari (2015) for data taken with SDO/AIA in the Fe XII $193 \AA$ channel (hereafter AIA $193 \AA$ ). The CBP identification was based on invariant Zernike moments and a 

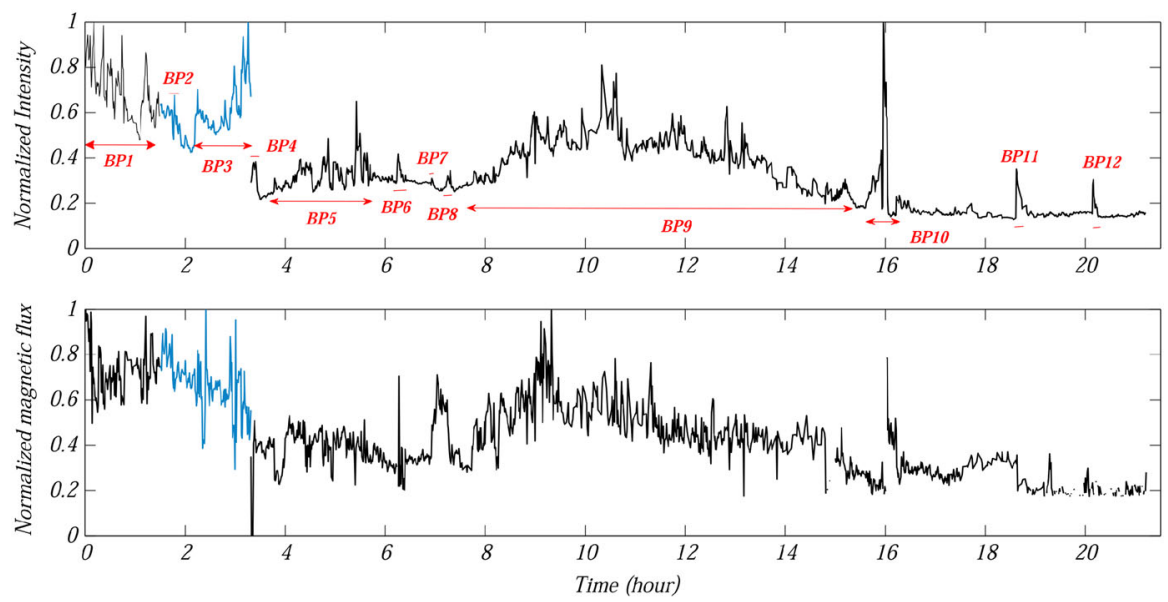

Fig. 7 CBP intensity lightcurves in SDO/AIA $193 \AA$ (top panel) and corresponding total magnetic flux lightcurve (bottom panel). Image reproduced with permission from Alipour and Safari (2015), copyright by AAS

machine learning method (the Support Vector Machine classifier). The method was set to identify CBPs with a size larger than $4 \mathrm{Mm}\left(6^{\prime \prime}\right)$ and smaller than $20 \mathrm{Mm}\left(56^{\prime \prime}\right)$ (diameter), and lifetimes as short as $2 \mathrm{~min}$. The study classified CBPs into two lifetime distribution groups, one of transient CBPs which is actually microflaring in CBPs (see Sect. 7.1 for more details), and one named as persistent events. In the transient group events, lifetimes were less than $20 \mathrm{~min}$, with an average of $12 \mathrm{~min}$. The persistent events had an average lifetime of $6 \mathrm{~h}$. The lifetime frequency distribution of CBPs was fitted with a linear power-law fit with a slope of $-1.6 \pm 0.07$ (see their Fig. 10). The relation of CBP lifetimes and their sizes shows that $95 \%$ of CBPs have lifetimes of less than $20 \mathrm{~h}$ and CBPs with shorter lifetimes $(<6 \mathrm{~h})$ are more scattered in size. CBPs with longer lifetimes appear to generally have larger sizes.

The automatic identification of CBPs can be challenging considering CBPs' dynamic activity. Furthermore, some CBPs result from more complicated bipolar magnetic flux distribution including the cases of CBPs formed from a flux convolution or a bipole emergence nearby pre-existing flux concentrations. In these cases the CBPs have more complex magnetic connectivities and it is hard to determine whether one or several CBPs are present at the studied location (Mou et al. 2016, 2018). The examination of Fig. 16 in the study of Alipour and Safari (2015) shows a case where 12 CBPs are selected (lifetimes from a few minutes to several hours) during $20 \mathrm{~h}$ of observations in a small region of $40^{\prime \prime} \times 40^{\prime \prime}$. The intensity variability possibly caused by microflarings and micro-eruptions in CBPs (Mou et al. 2018) has resulted in the identification of multiple CBPs rather than a dynamically evolving one or two CBPs due to more complex magnetic flux distribution (also see Fig. 7 and movie_1_alipour.mpg and movie_2_alipour.mpg in Alipour and Safari 2015).

To summarize, when observed in EUV at $\sim 1 \mathrm{MK}$, CBPs have lifetimes that are generally below $\sim 20 \mathrm{~h}$. They have shorter lifetimes in X-rays of $\sim 11 \mathrm{~h}$ on average. Longer living CBPs are found more often in active region latitudes, i.e., $\leq \pm 30^{\circ}$, and 
larger CBPs tend to have longer lifetimes. The lifetime range in both X-ray and EUV requires further examination using present data while accounting for the complexity and dynamics of the phenomena.

\subsection{Solar disk distribution and N-S hemisphere asymmetry}

The first X-ray observations of CBPs taken with the Skylab S-054 X-ray telescope revealed that XBPs have a uniform latitude distribution except for AR latitudes (Golub et al. 1974). They also showed that the longitudinal variation of XBPs at latitudes $> \pm 30^{\circ}$ is smaller than at low latitudes. Golub et al. (1975) updated their finding using $12 \mathrm{~h}$ synoptic S-054 observations. These data (the same dataset as in Golub et al. (1974) but a double image number) showed a latitude distribution that has two distinctive components, one uniform over a broad latitude range and one at active region latitudes, i.e., $\pm 30^{\circ}$ (Fig. 8). The ratio of the number of XBPs in the two hemispheres for two sets of longitudinal bins-one from $150^{\circ}$ to $330^{\circ}$ and the other from the two bins $0^{\circ}-150^{\circ}$ and $330^{\circ}-360^{\circ}$ — was found to be $2.4 \pm 0.2$ for latitudes of $< \pm 30^{\circ}$ and $1.5 \pm 0.2$ for the high latitude XBPs. The analysis of Skylab X-ray image datasets taken over a 6 month time period in 1973 (May-November) by Golub et al. (1976b) found that the variation of the number of high latitude XBPs is in phase with the low latitude variation reaching a maximum at the peak of the active region emergence. The XBP number at low latitudes was found to vary similarly to the active region occurrence rate. From SOHO/EIT observations, McIntosh and Gurman (2005) further confirmed the existence of AR and QS CBP distribution components.

Brajša et al. (2005) obtained a two component latitudinal distribution of CBPs using an interactive and an automatic identification procedure for data taken with the SOHO/EIT from 1998 June 4 until 1999 May 22. The interactive method (originally described in Brajša et al. 2001) was based on a visual computer screen tracking of CBPs in consecutive images. The automatic method (Brajša et al. 2001, 2002) employed a region-of-interest segmentation applied on triplets of images taken every $6 \mathrm{~h}$. It was set in a way that its parametrisation matches as close as possible the interactive method. One distribution component was found to be uniformly distributed to a latitude of $70^{\circ}$. The second is an overimposed component that has a maximum at central latitudes between $10^{\circ}$ and $20^{\circ}$ for the interactive method, and in the range from $10^{\circ}$ to $30^{\circ}$ for the automatic one. An asymmetry was found with more CBPs in the southern solar hemisphere than in the northern. This asymmetry was statistically significant only for the interactive but not for the automatic method. The authors comment that while Golub et al. (1975) found a second component starting at the equator to up to $\pm 30^{\circ}$, their study does not find a second component at equatorial latitudes. The low latitude CBP distribution component is known to be related to sunspot activity and as the two studies used data taken at different phases of the solar cycle activity, different results should be foreseen (Brajša et al. 2005). Golub et al. (1975) used data from the decline and close to the solar minimum phase of cycle 20 (May-June 1973), and therefore, CBPs related to ARs are expected to occur close to the equator. In contrast, Brajša et al. (2005) employed data from the start of the rising phase of cycle 24 (June 1998-May 1999) during which ARs emerged at latitudes higher than $\pm 20^{\circ}$. 

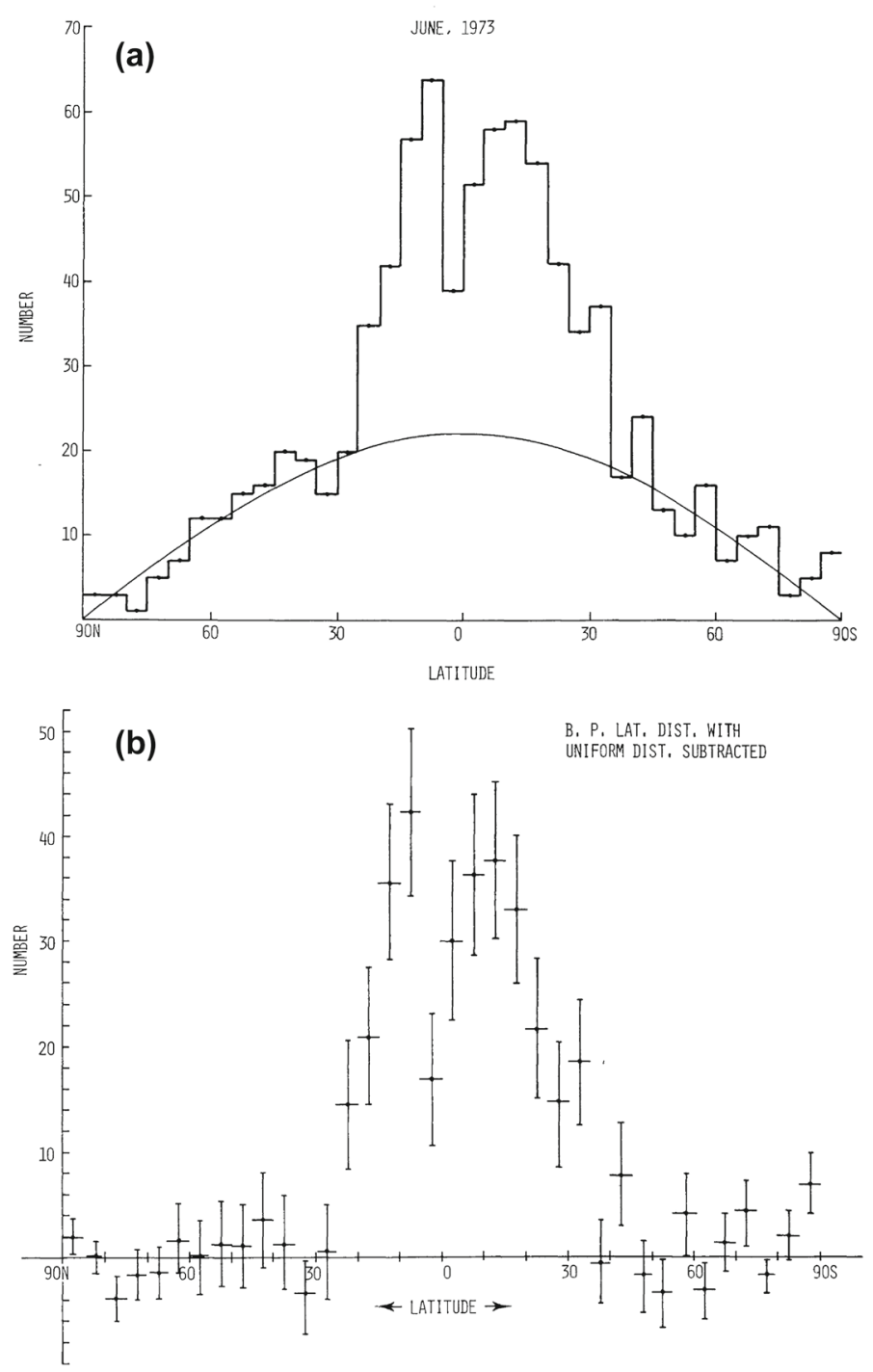

Fig. 8 Top panel: latitude distribution of XBPs. The solid line curve represents a uniform distribution. Bottom panel: latitude distribution with the uniform distribution fit from the top panel subtracted. Image reproduced with permission from Golub et al. (1975), copyright by Springer

In summary, CBPs display a two component latitude distribution, one uniform component up to $70^{\circ}$ and one at active region latitudes $\left(\leq \pm 30^{\circ}\right)$ that varies with the solar cycle activity. CBP numbers also show north-south asymmetry. Revisiting the subject of CBP number cycle variation by combining more than 2 decades of EUV observations will provide a better insight on the generation of solar activity given the presence of CBPs over large latitudes and during periods of low or no sunspot activity. 


\subsection{Sizes}

In X-ray observations, CBPs appear as compact roundish regions of enhanced X-ray emission with a typical diameter of $20^{\prime \prime}-30^{\prime \prime}$ and a brighter core of $5^{\prime \prime}-10^{\prime \prime}$, surrounded by diffused emission (Golub et al. 1977). There is no strictly established definition of what size a CBP must have to qualify as such. However, the close association of CBPs with supergranulation cells concerning both their lifetime (cells-average 1.6 days, CBPs -1 day) and size (cells-27.1 Mm or $\sim 10^{\prime \prime}-50^{\prime \prime}$ ) (Hirzberger et al. 2008) strongly suggests that CBP sizes in the range $\sim 10^{\prime \prime}-60^{\prime \prime}$ (also adopted in some studies as un upper limit) should be considered. CBPs appear smaller when they first form during flux emergence, then evolve until reaching their maximum size as well as intensity (Mou et al. 2018). They become small again at the end of their lifetime and CBPs as small as $5^{\prime \prime}$ can be identified. The upper limit of CBP sizes could also be determined by the total bipolar magnetic flux. Ephemeral region total unsigned flux (up to $50 \%$ of CBPs relate to ERs, see Sect. 4 for more details) ranges from $\sim 3.0 \times 10^{18}$ to $\sim 10^{20} \mathrm{Mx}$, but no larger than $3 \times 10^{20}$ (Yang and Zhang 2014; van Driel-Gesztelyi and Green 2015, and the references therein). Therefore, $\sim 10^{20} \mathrm{Mx}$ should eventually be considered as an upper limit as flux above this value is presently considered as small active regions (pores) (van Driel-Gesztelyi and Green 2015, and the references therein).

From the analysis of $41 \mathrm{CBPs}$ in the SOHO/EIT $195 \AA$ passband, Zhang et al. (2001) reported an average size of $110 \mathrm{Mm}^{2}$, or $14^{\prime \prime} \times 14^{\prime \prime}$. The survey by Longcope et al. (2001) of 285 CBP observed in SOHO/EIT $195 \AA$ images found sizes ranging from 4 to $40 \mathrm{Mm}$. Recently, Alipour and Safari (2015) determined a CBP average size of $130 \mathrm{Mm}^{2}$, or $15^{\prime \prime} \times 15^{\prime \prime}$, however a criterion of maximum size of $\leq 56^{\prime \prime}$ (diameter) for the automatic identification was applied which eliminated larger CBPs.

To conclude, there is no strict definition of the CBP size range, but bright loop complexes that connect magnetic bipoles with a total flux strength of up to $\sim 10^{20} \mathrm{Mx}$ and diameter ranging from $5^{\prime \prime}$ to $60^{\prime \prime}$ could be assumed as typical for CBPs.

\subsection{Heights}

The heights of CBPs were generally estimated from the emission in coronal spectral lines or imaging channels. The first study doing so is the one by Simon and Noyes (1972) who used spectroheliograms taken with the Harvard experiment on the Orbital Solar Observatory IV (OSO-IV). CBPs only in ARs were used to estimate the height formation of 15 spectral lines above the photosphere with formation temperatures from $\log T(\mathrm{~K})=4.0$ (Lyman lines) to $\log T(\mathrm{~K})=6.6$ (Fe XVI). Apart from the CII line which gives an anomalous height of $\sim 10,000 \mathrm{~km}$, the height measurement of the coronal lines that relate to CBPs heights are: in Ne VIII $770 \AA$ $8800 \pm 1400$ km, MgX $625 \AA-10,600 \pm 1600$ km, Si XII $499 \AA-9400 \pm 1700$ km, Fe XV $417 \AA-12,800 \pm 2200 \mathrm{~km}$, and FeXVI-15,200土2300 km. The emission in the lower temperature lines (chromospheric or low transition-region) are found to be $\sim 3000 \mathrm{~km}$, estimated with errors of $\sim 50 \%$. This emission originates in the legs 

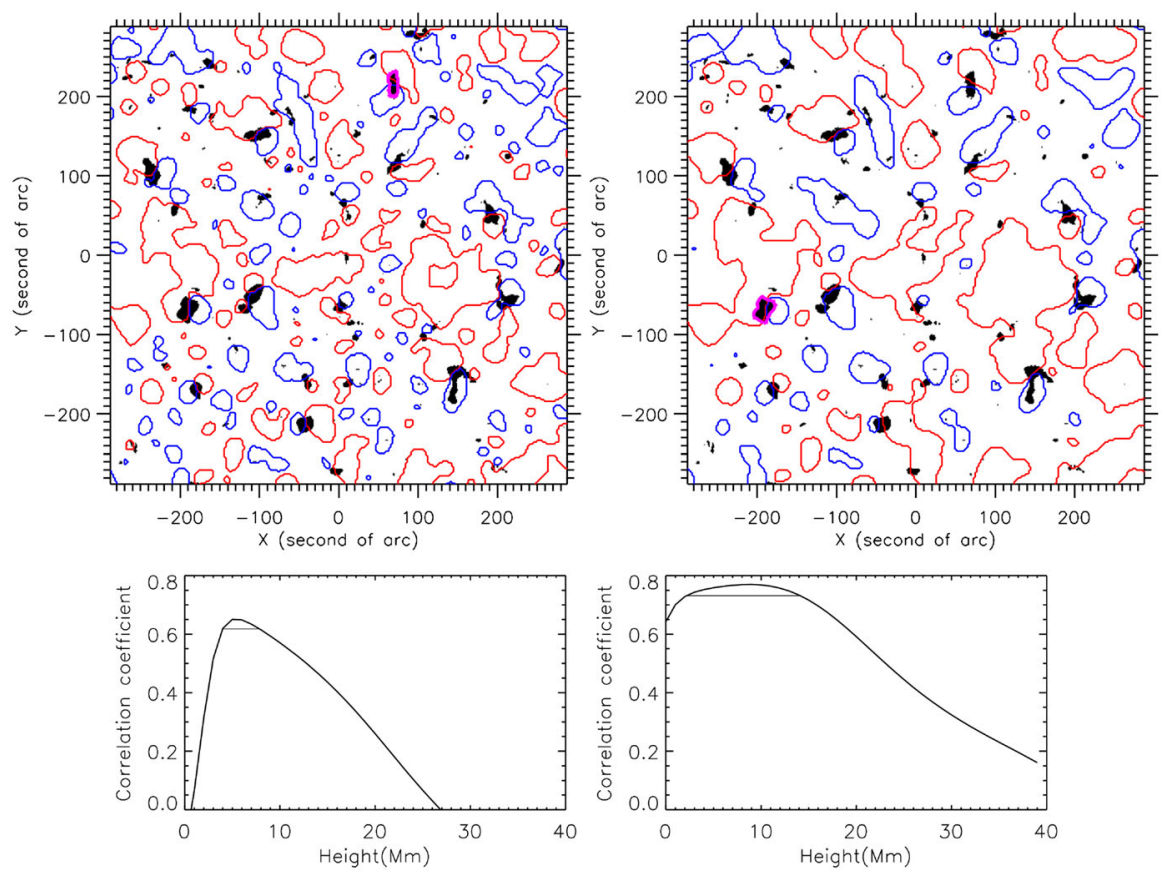

Fig. 9 Top panel: CBPs in SOHO/EIT $195 \AA$ (black areas) overlaid map of magnetic polarities at $5 \mathrm{Mm}$ (left-hand panel) and $9 \mathrm{Mm}$ (right-hand panel) heights in the solar atmosphere. Red contours correspond to negative polarities, while blue to positive. Bottom panel: correlation coefficients of the horizontal component of the magnetic field and the square root of the FeXII intensity enhancement for the two purple colour outlined CBPs shown in the top panel. Image reproduced with permission from Tian et al. (2007), copyright by COSPAR

of the CBP loops. All follow-up studies only used coronal imaging data including SOHO/EIT, SECCHI/EUVI, and SDO/AIA.

By studying the solar differential rotation using CBPs as tracers, Brajša et al. (2004) also determined CBP heights. Two methods were used, interactive and automatic (for details see Sect. 3.3), to identify CBPs in the SOHO/EIT $195 \AA$ passband. The used methodology is originally described in Rosa et al. (1998). The averaged heights above the photosphere for the interactive methods were estimated at $9900 \mathrm{~km}$ and for the automatic-11,600 km. Sudar et al. (2016) using the same method estimated the average CBP heights identified in SDO/AIA $193 \AA$ A passband images at $\sim 6500 \mathrm{~km}$.

Tian et al. (2007) combined the radiance of CBP in Fe XII $195 \AA$ images taken with SOHO/EIT and magnetic field extrapolation based on Kitt Peak longitudinal magnetograms to evaluate the heights of the Fe XII emission in CBPs. Linear forcefree extrapolations from the photosphere to $80 \mathrm{Mm}$ height were used to obtain the three components of the magnetic field vector B. The correlation coefficient between the maximum of the Fe XII intensity enhancement and the horizontal component of the extrapolated magnetic field vector at the same $x-y$ position in planes of different heights was defined as the correlation height (Fig. 9). It was determined that for the majority of the analysed CBPs the $195 \AA$ emission occurs below 20,000 km with an 
average height of $5000 \mathrm{~km}$. Most of the CBPs seen in the Fe XII passband were located on top of the associated magnetic loop.

Kwon et al. (2010) developed a three-dimensional reconstruction method for pointlike features (e.g., CBPs) based on the principle that the position of a point in a threedimensional space is specified as the intersection of two lines-of-sights. Data taken with the Sun Earth Connection Coronal and Heliospheric Investigation (SECCHI) Extreme-ultraviolet Imagers (EUVI) on board the twin STEREO A and B spacecrafts in the $171 \AA, 195 \AA, 304 \AA$ and $284 \AA$ passbands were used to study the heights of 210 CBPs. The study found that in the $171 \AA[\log T(\mathrm{~K}) \sim 5.9], 195 \AA[\log T(\mathrm{~K}) \sim$ 6.1] and $284 \AA[\log T(\mathrm{~K}) \sim 6.3]$ passbands CBPs have average heights of 5100, 6700 and $6100 \mathrm{~km}$, respectively. They also determined that the CBP emission in the $304 \AA$ passband is at around $4400 \mathrm{~km}$ and is associated with the legs of the coronal loops forming the CBPs rather than their tops. In a follow-up study, Kwon et al. (2012) studied the temporal evolution of CBP heights and lengths using the same method. From the analysis of three CBPs three distinctive changes of CBP heights and lengths were found, including decreasing, increasing and steady, that correspond to three specific motions of the magnetic photospheric flux concentrations, converging, diverging and shearing, respectively. The study concluded that CBPs represent multitemperature loop systems composed of hot loops $[\log T(\mathrm{~K}) \sim 6.2]$ overlying cooler ones $[\log T(\mathrm{~K}) \sim 6.0]$ with cool legs $[\log T(\mathrm{~K}) \sim 4.9]$.

To summarise, CBPs heights at coronal temperature observations $(\sim 1.0 \mathrm{MK})$ are found to be in the range from 5000 to $10,000 \mathrm{~km}$. An average height of $6500 \mathrm{~km}$ should be expected at a lifetime peak. It is important to note that chromospheric and transition region emission provides information only for the CBP loop footpoints and legs, and occasionally may originate from low-lying or newly emerging CBP loops.

\subsection{Occurrence rate}

Various methods have been used to determine the occurrence rate of CBPs. From the visual inspection of Skylab X-ray images Golub et al. (1974) ascertained that at least 200 XBPs appear at any time on the whole Sun (100 XBPs on the visible disk). They estimated an occurrence rate of 1500 XBPs emerging per day. Also from the visual inspection of EUV images taken with SOHO/EIT, Longcope et al. (2001) evaluated an occurrence rate of $396 \mathrm{CBPs}$ (density of $1.3 \times 10^{-4} \mathrm{Mm}^{-2}$ ) per disk image similar to the number obtained by Golub et al. (1976b) of 200-660 (0.6-2.0 $\left.\times 10^{-4} \mathrm{Mm}^{-2}\right)$ in Skylab X-ray data.

An automatic algorithm based on a local intensity threshold as well as CBP sizes and shapes was employed by Hara and Nakakubo-Morimoto (2003) that estimated a density of XBPs in Yohkoh/SXT data at $0.2-0.7 \times 10^{-4} \mathrm{Mm}^{-2}$. McIntosh and Gurman (2005) applied a variation of this algorithm on CBPs in EUV data obtained with SOHO/EIT in the $171 \AA, 193 \AA$, and $284 \AA$ channels taken over 9 years. The CBP number in the FeXV $284 \AA$ channel was similar to those obtained earlier by Hara and Nakakubo-Morimoto (2003) ( 150) in soft X-rays. The CBP number was found to increase when detected in low temperature emission, with almost 100 more in SOHO/EIT $195 \AA(\sim 250)$ and $\sim 150$ more in SOHO/EIT $171 \AA(\sim 300)$ (see Fig. 10). The studies of Hara and Nakakubo-Morimoto (2003) and McIntosh and 
Fig. 10 Variation of the CBP number in the SOHO/EIT 171, 195 and $284 \AA$ passbands from 1996 to 2004. Each dot represents the daily average CBP number. The orange colour denotes the co-temporal CBPs in all three EUV channels. Image reproduced with permission from McIntosh and Gurman (2005), copyright by Springer

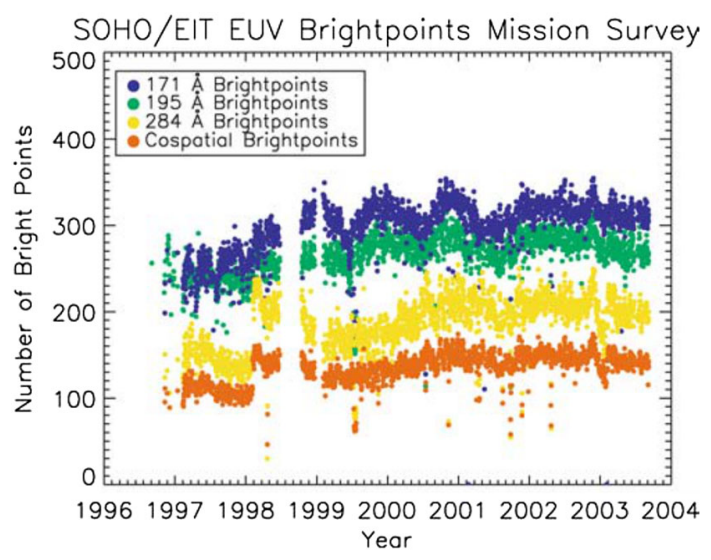

Gurman (2005) both obtained an occurrence rate lower than in other studies which is possibly related to the used algorithm that discriminates CBPs with a certain shape and size. Sattarov et al. (2010) estimated from SOHO/EIT $195 \AA$ images a higher range of 280-450 CBPs.

Most recently, Alipour and Safari (2015) used an automatic detection method based on a machine-learning technique and Zernike image moments on SDO/AIA $193 \AA$ images taken during a 4.4-year period (from 2010 June 1 to 2014 October 31) to investigate the statistical properties of CBPs. The study reports that the CBPs average number and mean density (per full disk image) are 572 (ranging from 427 to 790) and $1.9 \times 10^{-4} \mathrm{Mm}^{-2}\left(1.39 \times 10^{-4} \mathrm{Mm}^{-2}\right.$ to $\left.2.56 \times 10^{-4} \mathrm{Mm}^{-2}\right)$, respectively. This is a the highest number of CBPs ever detected, far larger than the numbers obtained by McIntosh and Gurman (2005) and Sattarov et al. (2010) in SOHO/EIT data in the same passband. One possible explanation is the use of a different identification method. By applying their identification method on SOHO/EIT $195 \AA$ data, Alipour and Safari (2015) concluded that $8 \%$ more CBPs are identified in SDO/AIA $193 \AA$ data than in SOHO/EIT $195 \AA$ (Fig. 11), possibly caused by the higher spatial resolution of SDO/AIA which helps to identify smaller size CBPs.

To summarise, the daily average number of CBPs in the EUV $193 \AA$ passband is presently estimated at $\sim 570$, with a range from 427 to 790 . The average CBP number density is $1.9 \times 10^{-4} \mathrm{Mm}^{-2}\left(1.39 \times 10^{-4} \mathrm{Mm}^{-2}\right.$ to $\left.2.56 \times 10^{-4} \mathrm{Mm}^{-2}\right)$. The number of CBPs changes when observed in passbands with different temperature response with the largest number found in $171 \AA$ passbands and the lowest in $284 \AA$ and X-rays, with a difference of up to 150 CBPs. It is important to note that the number of identified CBPs will depends of various factors including spatial resolution, photon statistics, exposure duration when data from the same instrument are compared, etc. Therefore, comparing the statistics between different studies should consider these factors.

\subsection{Solar cycle variation}

By comparing the number of XBPs detected in 1976 (during two rocket flights) with XBP numbers in 1973 (Skylab), Davis et al. (1977) established that the CBP number 
Fig. 11 SOHO/EIT full disk image in the Fe XII $195 \AA$ passband taken on 2008 February 16 00:00 UT. The locations of 670 CBPs are outlined with green contours. Image reproduced with permission from Alipour and Safari (2015), copyright by AAS

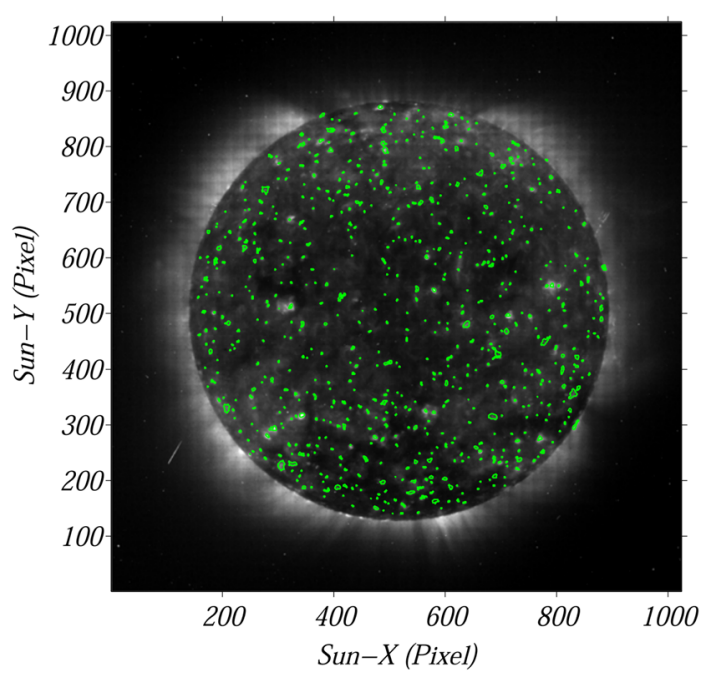

Fig. 12 CBP number variation with the solar cycle. Image reproduced with permission from Golub et al. (1979), copyright by AAS

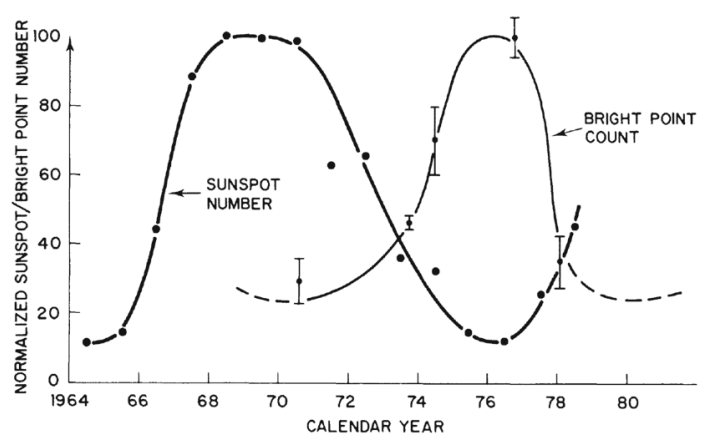

in X-rays has increased by a factor of 2 in a three year time interval (from the declining to minimum phase of the solar cycle). In the same period of time the sunspot number has decreased by a factor of 3 . A conclusion was reached that the short-lifetime end of the magnetic flux spectrum associated with XBPs is out of phase with the solar sunspot cycle. This finding was later confirmed by Golub et al. (1979) from data taken from 1970 to 1978 that cover the time period near the peak of cycle 20 and the following decline phase. A clear anticorrelation was found between the XBPs number and sunspot number (Fig. 12). It was concluded that there exists a secondary cycle that operates in anti-phase with the primary (active region) solar cycle. The result was later confirmed by Davis (1983) and Harvey (1985) who used data that extended into solar cycle 21. Based on the study by Giovanelli (1982) on the role of unipolar and mixed polarity fields, Harvey (1985) suggested that the inverse correlation is related to the higher probability of chance encounters between magnetic fluxes of opposite polarity during low levels of solar activity rather than flux emergence.

To investigate this anticorrelation, Nakakubo and Hara (2000) applied an automatic algorithm to identify XBPs in Yohkoh/SXT images covering the time period from December 1992 to August 1997. An XBP was defined as a small region of enhancement 
Fig. 13 Top two rows: total number of XBPs as a function of time (a), latitude versus time distribution of XBP numbers (b), and c fractional distribution of XBPs with solar latitude. Bottom two rows: the same as the top rows but for photospheric magnetic bipoles. The vertical dashed line in a separate Fe I $5507 \AA$ (left) and Fe I $8688.6 \AA$ (right). Solid line in $\mathbf{c}$ is a least-square fit by Gaussian function. Image reproduced with permission from Sattarov et al. (2002), copyright by AAS
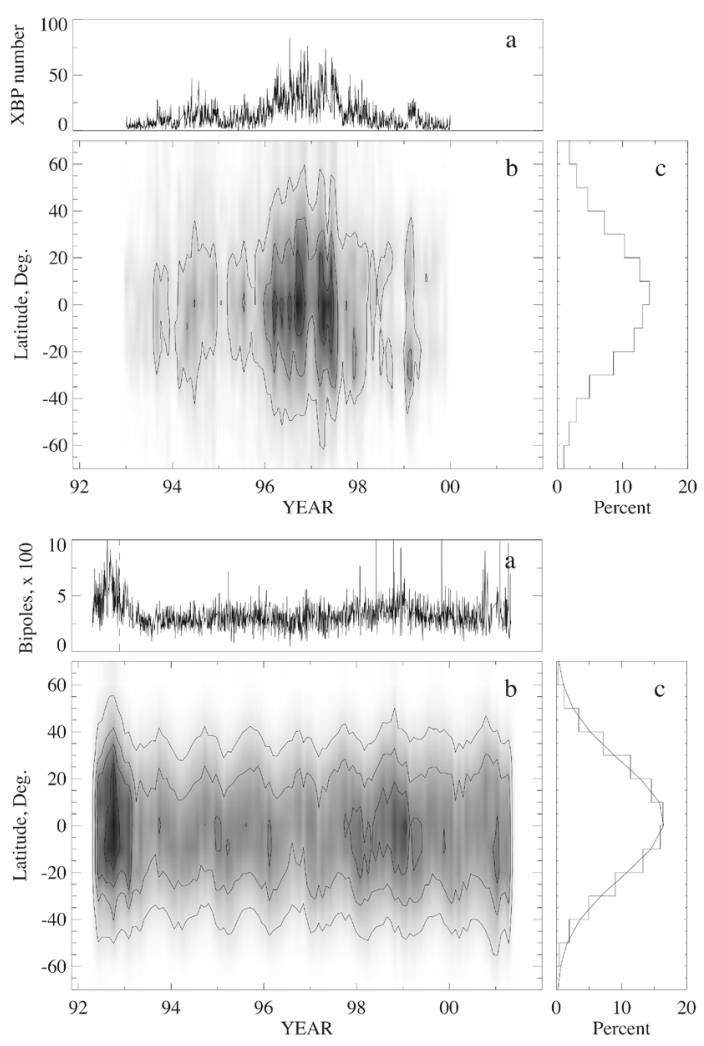

$\mathrm{X}$-ray emission with respect to the background emission determined from an adjacent region. This approach eliminated the effect of a variable background emission, i.e., higher background in ARs and lower in CHs. The study found that after 1995 the XBP number varied inversely with the sunspot number and reached maximum during the solar minimum. However, the XBP and background intensity distributions suggested that the XBP number increase near the solar minimum is due to a decrease of the background intensity which permits the identification of fainter CBPs.

Sattarov et al. (2002) compiled a survey of the occurrence rate of CBPs in X-rays from 1993 to 2000 using Yohkoh/SXT full-disk images that cover the decline phase of cycle 22 and the rising phase of cycle 23. Longitudinal magnetograms from the Kitt Peak National observatory were also used to identify bipolar regions with magnetic field strength $>20 \mathrm{G}$ and magnetic fragment sizes ranging between $5^{\prime \prime}$ and $55.2^{\prime \prime}$. The study confirmed the already known anti-cycle correlation of CBPs. While the bipole numbers remained unchanged during the studied period ( $\sim 250$ per full-disk image), the CBP numbers varied changing to $\sim 10$ per image from 1993 to 1995 (decline phase), reaching up to $\sim 50$ between 1996 and 1998 (minimum phase). The numbers went to become smaller after 1998 during the rising phase of cycle 23 (Fig. 13). Based on the expectation that the percentage of XBPs associated with magnetic bipoles should not vary, the authors concluded that the variation of identified XBPs is caused 
by background intensity variations due to the presence of active regions, i.e., it is purely a visibility effect. The latitude distribution of XBPs, however, showed a clear excess of XBPs at active region latitudes $\pm 30^{\circ}$ despite the increased background intensity there. The study suggests that CBPs that form in the sunspot belts may have been formed by magnetic activity that is different from QS CBPs, i.e., are related to ephemeral active regions whose latitude distribution follows the Spörer law. At the same time they note that AR-belt CBPs show, however, the same properties as QS CBPs.

Hara and Nakakubo-Morimoto (2003) continued their preliminary study (Nakakubo and Hara 2000) by analysing the XBP number variation in data from Yohkoh/SXT taken during the time period 1993-2000. Composite images that consist of Yohkoh/ SXT images taken within $30 \mathrm{~min}$ with three different exposures of 30, 5.3, and $0.17 \mathrm{~s}$ were used for which the saturated area in the images taken with $30 \mathrm{~s}$ and $5.3 \mathrm{~s}$ caused by the presence of bright active regions were corrected. The unit of soft-X-ray intensity $I$ in a single pixel of the composite image is DN pixel ${ }^{-1} \mathrm{~s}^{-1}$ in which DN equals $365 \mathrm{eV}$ in X-ray photon energy for an Yohkoh/SXT image pixel with a size of 4.9" $\times 4.9^{\prime \prime}$. The detection method is described in detail in section 3 of their paper. The XBPs were selected as significant brightenings with respect to the adjacent background corona (including existing active regions) with sizes of $<60^{\prime \prime}$. The authors investigated how the CBP number changes in the dark (with background intensities $I_{\text {back }}<10^{0.8}$ ) and bright $\left(10^{0.8}<I_{\text {back }}<10^{1.6}\right)$ regions of the quiet Sun. The number of all CBPs showed a certain increase during solar minimum when (Fig. 14a) a correction for the obscuration by bright active regions was not applied. In dark areas (e.g., CHs), the CBP number clearly anticorrelates with the sunspot cycle (Fig. 14b). In contrast, the bright region CBP numbers follow the solar cycle trend (Fig. 14c). The fractional areas shown in Fig. 14d and the effect of the background emission explain to a large extend the presence of the anti-correlation cycle in CBPs numbers.

Interesting studies by McIntosh et al. (2014a, b) have found that CBPs preferentially appear at locations that mark a giant convective scale on photospheric magnetism with sizes in the range from 100 to $250 \mathrm{Mm}$, named by the authors as "g-nodes". This scale is one of four scales (the rest are a megameter scale related to granulation, a few tens of megameters - super-granulation, and many hundreds to thousands of megameters linked to coronal holes and active regions) identified by applying a magnetic range of influence (MRoI) that is a measure of a length over which the photospheric lineof-sight magnetic field is (for more details see McIntosh et al. 2014b). By combining SDO/AIA coronal imaging data and identification maps of g-nodes, it was found that CBPs are preferentially located at g-nodes. However, not all g-nodes were related to CBPs identified in the SDO/AIA $193 \AA$ channel. The follow-up work by McIntosh et al. (2014a) concluded that small-scale emergence activity related to CBPs and gnodes (only the ones forming at AR latitudes $\leq 30^{\circ}$, see Sect. 3.3) could be used to forecast the solar cycle (see Fig. 17 of their paper).

To summarise, the number of CBPs outside the active region belt are found to remain relatively constant during the solar cycle. Background emission variation was established as the main cause for the solar cycle anti-correlation usually observed. The CBP number in AR latitudes is found to change with the solar cycle suggesting a relation to ephemeral regions in the AR belt. 

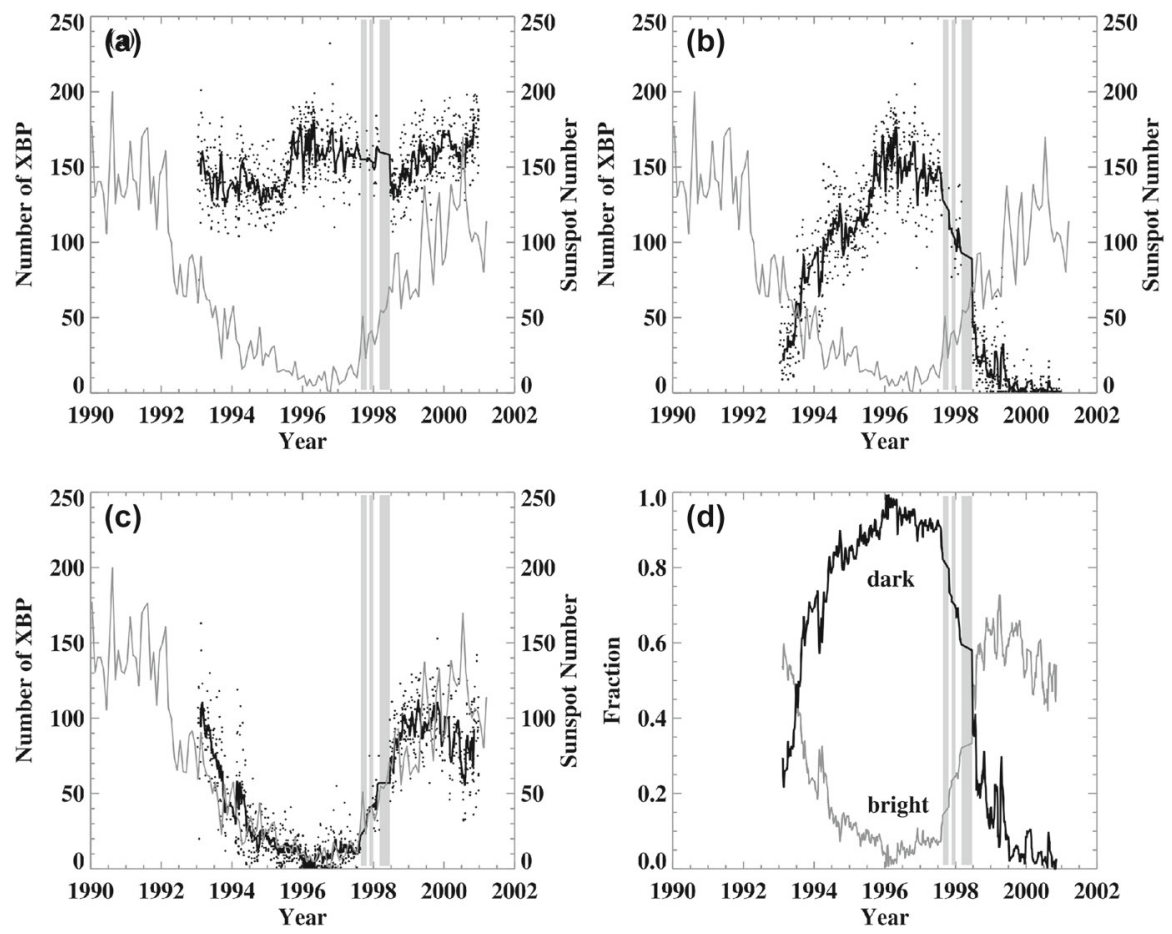

Fig. 14 a Number of XBPs in an X-ray composite image. Dots and a thick solid line represent the 10 days' running mean. The grey vertical stripes indicate periods of no data with $30 \mathrm{~s}$ exposures. b Number of detected XBP in an area with a background X-ray intensity of $I_{\text {back }}<10^{0.8} \mathrm{SXU}$, and c of $10^{0.8} \leq$ $I_{\text {back }}<10^{1.6} \mathrm{SXU}$ shown with thick lines. d Fractional areas occupied by dark $\left(I_{\text {back }}<10^{0.8} \mathrm{SXU}\right)$ and bright $\left(10^{0.8} \leq I_{\text {back }}<10^{1.6} \mathrm{SXU}\right)$ given with thick and thin solid lines. In all panels the monthly averaged sunspot number is given with a thin solid line. Image reproduced with permission from Hara and Nakakubo-Morimoto (2003), copyright by AAS

\section{Magnetic properties}

\subsection{Photospheric magnetic fields}

Knowledge on the magnetic field configuration of any solar phenomenon is crucial for deriving realistic models of the physical processes that generate, sustain or destroy them. When the very first solar X-ray observations were coupled with photospheric longitudinal magnetic field images, they revealed a clear association of the observed $\mathrm{X}$-ray structures with underlying photospheric flux concentrations. Among them Xray bright point-like sources, i.e., CBPs, outside active region belts were found to overlay small-scale bipolar magnetic flux concentrations (Krieger et al. 1971; Harvey et al. 1975).

Golub et al. (1977) made the first detailed investigation of the CBP magnetic properties. The study compares ephemeral active regions (ER) and XBPs in simultaneously obtained Kitt Peak National Observatory magnetograms and Skylab X-ray images to answer a range of qualitative and quantitative questions concerning the bipolarity of 
Fig. 15 Top panel: Kitt Peak longitudinal magnetogram. Bottom panel: CBPs in X-rays. The CBPs and their corresponding bipoles are marked with solid circles. Also shown are dashed-line circles indicating bipolar regions not related to XBPs. Image reproduced with permission from Golub et al. (1977), copyright by Springer

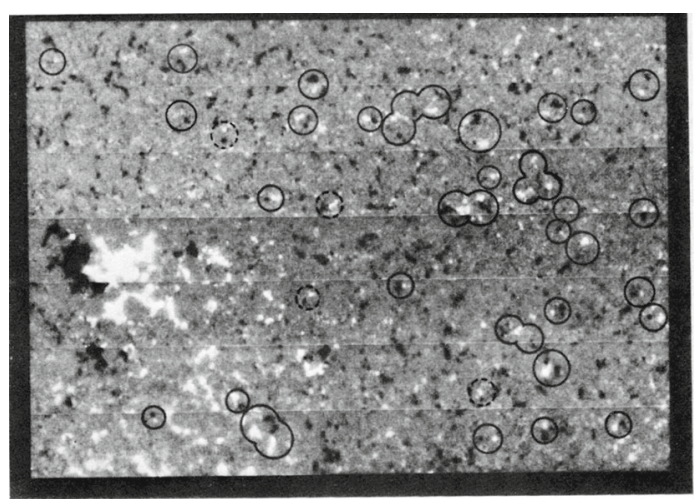

1520-1620 KPNO

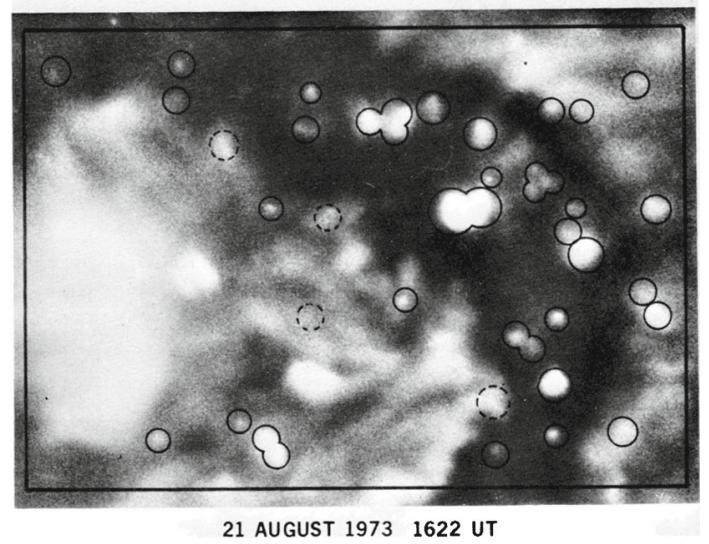

XBPs, their relation to flux emergence, and the CBP contribution to the total emerging flux on the Sun. The term ER was first introduced by Dodson (1953) to describe a newly emerging bipolar region. Harvey and Martin (1973) and Harvey et al. (1975) defined ERs as small bipolar regions (size of $\sim 40^{\prime \prime}$ ) with a total magnetic flux of $\sim 10^{20} \mathrm{Mx}$ that is now known to be as low as $10^{16} \mathrm{Mx}$ (Guglielmino et al. 2012, and the references therein). ERs have an average lifetime of less than a day appearing bright in $\mathrm{H} \alpha$. Golub et al. (1977) determined that all XBPs were associated with bipolar magnetic features (Fig. 15), except for the very newly emerged or old and decaying XBPs which usually have weaker and dispersed fields that were possibly below the resolving power of the observing instrument at that time. However, only $49 \%$ of the ERs were found to be linked to XBPs (36 out of 73 XBPs). A large part of the remaining ERs were associated with diffuse X-ray brightenings overlaid by large coronal structures that possibly prevented the identification of these brightenings as CBPs. Tang et al. (1982) also found no one-to-one correspondence of ERs and CBPs at transition region and coronal temperatures, with more ERs than CBPs present at any given time. Later studies confirmed that strong background emission may prevent the identification of CBPs (for details see Sect. 3.6). The study also suggests that the partial correspondence to ERs may be related to the evolutionary history of XBPs, 
i.e., only during a short period (second and third quarter) of their lifetime CBPs can reach X-ray temperatures (see their Table 1) which was later confirmed from EUV and X-ray co-observations (Zhang et al. 2001, and Fig. 5). Golub et al. (1977) found that the total magnetic flux in an ER is an increasing function of the CBP age and determined that the age of XBPs is linearly correlated with the separation distance of the associated bipolar structure with a growth rate of $2.2 \pm 0.4 \mathrm{~km} \mathrm{~s}^{-1}$.

A few years later, Harvey (1985) reported a study of 'dark points' in the HeI $10,830 \AA$, known to be a counterpart of CBPs, and their associated magnetic bipoles for data taken over an $\sim 11$-year period. Their results showed that one third of the dark points are associated with ephemeral regions while at least two thirds result from the chance encounter of existing opposite polarities. The flux disappearance during this polarity interaction made the author suggest that CBPs are driven by magnetic reconnection in the solar corona, and the flux chance encounter is the mechanism for the flux removal in the quiet Sun. Harvey (1984) and Harvey et al. (1994) established that $70-80 \%$ of XBPs are associated with the chance encounter of network flux and only $20-30 \%$ are related to newly emerging bipolar fluxes, i.e., ERs.

The total magnetic flux of CBPs was estimated in the range of $10^{19}-10^{20} \mathrm{Mx}$ (Krieger et al. 1971; Golub et al. 1974; Harvey et al. 1975), with most typical values of $2-5 \times 10^{19} \mathrm{Mx}$. Golub et al. (1976b) suggested that the total magnetic flux emerging on the Sun per day associated with X-ray BPs ranges between $1.2 \times 10^{22}$ and $3.6 \times$ $10^{22} \mathrm{Mx}$. If only high $\left(> \pm 30^{\circ}\right)$ latitudes are considered, the emerged total flux is then between $6 \times 10^{21} \mathrm{Mx}$ and $1.2 \times 10^{22} \mathrm{Mx}$ per day. This evaluation assumes that CBPs are entirely related to only flux emergence. A typical XBP associated with an ER that has a growth rate of $1.5 \times 10^{19} \mathrm{Mx} \mathrm{s}^{-1}$ (data noise level of $10 \mathrm{G}$ ) and for a lifetime of $8 \mathrm{~h}$ reaches a total unsigned flux of $2.0 \times 10^{19} \mathrm{Mx}$ (Golub et al. 1977). Similarly to active regions the bipolar flux evolves with an initial rapid flux emergence followed by a more gradual decay phase.

Preś and Phillips (1999) studied the full lifetime of several CBPs in the QS using SOHO/EIT and MDI data. They found that the CBP emission correlates with the variation of the associated total magnetic flux that was later confirmed by Madjarska et al. (2003) from SOHO/EIT and MDI data and Chandrashekhar et al. (2013) from SDO/AIA and HMI data. Preś and Phillips (1999) estimated the total magnetic flux at the CBP peak intensity for one of the observed CBPs at $5.0 \times 10^{19} \mathrm{Mx}$ (see their Fig. 2), while Madjarska et al. (2003) obtained $2.0 \times 10^{20} \mathrm{Mx}$ at the maximum intensity of the studied CBP also from SOHO/MDI data. Similar values are found by Kwon et al. (2012). The analysis of a large set of CBPs analysed by Longcope et al. (2001) gives a median flux of $1.3 \times 10^{19} \mathrm{Mx}$.

Webb et al. (1993) analysed full-disk soft X-ray images from rocket flights, full disk magnetograms and He I 10,830 ̊ images from the Kitt Peak National Observatory, obtained before and after each flight, and BBSO magnetograms at more than $15 \mathrm{~min}$ cadence. The study was motivated by the fact that some of the earlier observations lacked co-temporality, and with the objective to resolve the debate on what photospheric magnetic field evolution is responsible for the CBP formation, evolution and decay. In total 165 XBPs were studied with 25 from BBSO and 140 in full-disk Kitt Peak magnetograms. The XBPs were more often associated with pre-existing magnetic features of opposite polarity that cancel with pre-existing or newly emerging 
flux. They showed a correspondence to network, intranetwork and ephemeral magnetic fluxes. Most importantly $88 \%$ of the XBPs were associated with converging magnetic flux of opposite polarities with the XBPs present before the onset of cancellation. Approximately $80 \%$ of the XBPs overlaid cancelling magnetic fluxes or older ephemeral regions. Young ephemeral regions were not found to have XBPs.

Harvey et al. (1994) reported the first study on simultaneous time sequences of longitudinal magnetic field data taken at the Kitt Peak National Observatory and Yohkoh/SXT images concluding that the relation of XBPs and magnetic field is more complex than previously thought. Two-thirds of all magnetic bipoles appeared not to be related to XBPs. The authors concluded that "emergence and cancelation of magnetic flux in the photosphere is not in itself a necessary and sufficient condition for the occurrence of an XBP. Rather, it is the interaction and reconnection of magnetic field with the existing, overlying magnetic field configuration that results in the occurrence and variability of XBPs."

A case study of a CBP photospheric field evolution using TRACE and SOHO/MDI data during its full lifetime is reported by Brown et al. (2001). The authors found that the bipolar region associated with the CBP formed from the coalescence of newly emerged and preexisting magnetic flux concentrations. Half way through the lifetime of the CBP, the loop structure appears to have twisted forming a sigmoid as a response of the footpoints' rotation (see Figs. 2 and 3 in their paper). A material ejection from the CBP may have taken place after the sigmoid formation but there is no clear evidence for it, probably because of the strong background emission. Madjarska et al. (2003) found that the CBP disappeared at coronal temperatures after a full cancellation of one of the magnetic polarities (see Fig. 16). Only one more study by Mandrini et al. (2005) reports a CBP evolving into a sigmoid-like structure followed by an eruption (see Figs. 3 and 4 in their paper).

Using a triangulation method applied on a time series of imaging data taken by SECCHI/EUVI on board the STEREO twin spacecraft, Kwon et al. (2012) studied the temporal evolution of the height and the length of 13 CBPs. Height and length decrease and increase, and steady values were associated with converging, diverging and shearing of the bipole flux concentrations. The two parameters that describe the 3D magnetic structure of the CBPs are well correlated with the separation distance of two opposite magnetic flux concentrations. However, no relationship was found between the CBPs' heights and lengths and the amount of magnetic fluxes. Mou et al. (2016) studied the photospheric magnetic field evolution of CBPs in the quiet Sun in data taken by SDO/AIA in the $193 \AA$ channel together with co-temporal SDO/HMI longitudinal magnetic field co-observations. The formation of the CBPs was found to involve emergence (i.e., ephemeral regions), convergence and coalescence. In total 70 BPs were randomly selected in SDO/AIA 193 Å images. Bipolar flux emergence (i.e., an ephemeral region) was identified in $50 \%$ of the cases. In 28 CBPs convergence of at least one polarity is found to proceed to a BP formation, while in only 6 cases coalescence of a small-scale field results in a CBP formation. Magnetic flux cancellation is found in all 70 CBPs. It involves the main polarities associated with the CBPs but also weak flux concentrations, generally confirming the studies by Golub et al. (1977), Harvey et al. (1994), and Webb et al. (1993). 

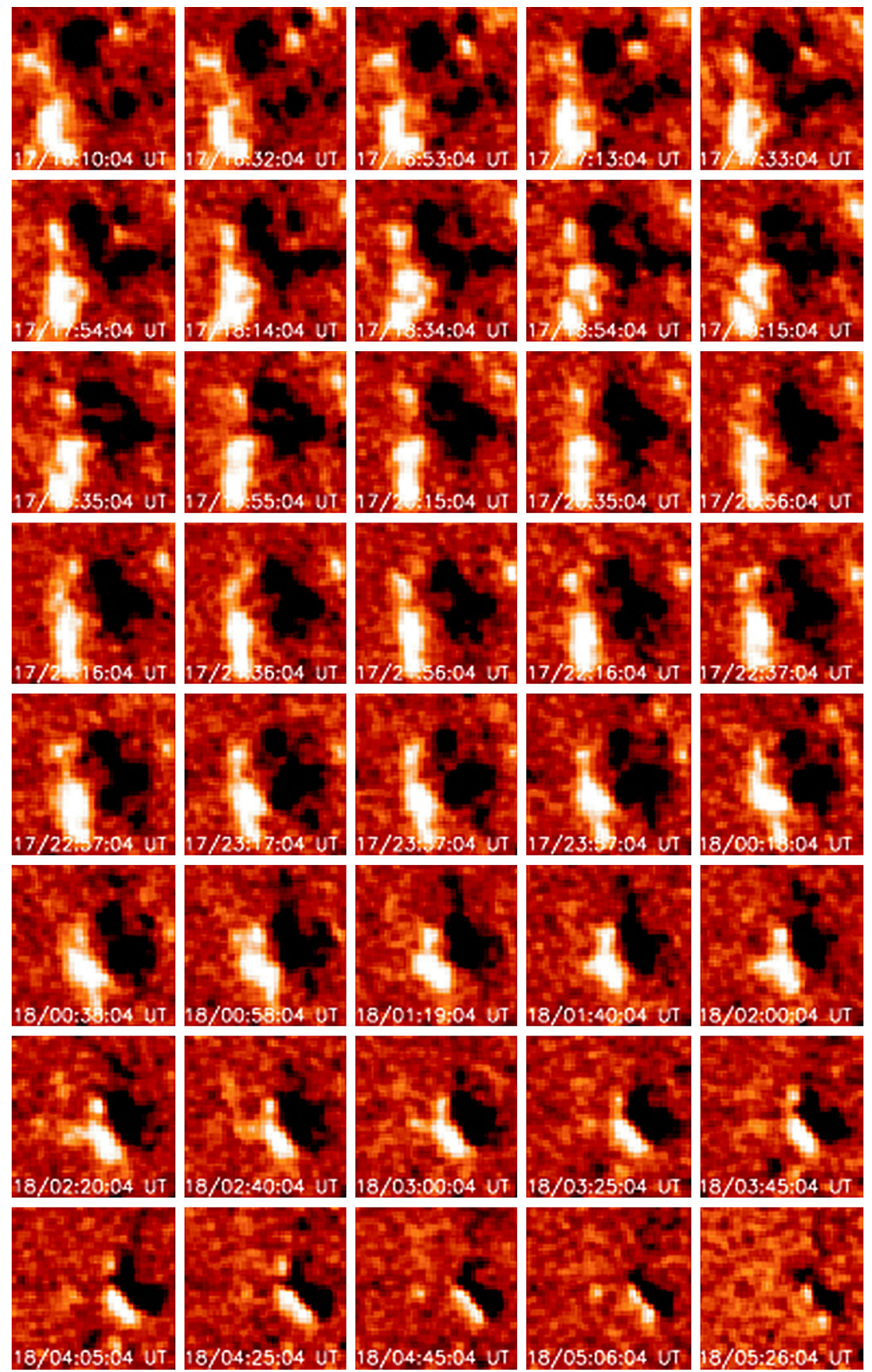

Fig. $16 \mathrm{SOHO} / \mathrm{MDI}$ images of the photospheric longitudinal magnetic field evolution (scaled from -25 to $25 \mathrm{G}$ ) of a magnetic bipole associated with a CBP observed in SOHO/EIT $195 \AA$ Å. Image reproduced with permission from Madjarska et al. (2003), copyright by ESO 
McIntosh (2007) applied the magnetic range of influence definition (MRoI, see Sect. 3.7 for more details) on SOHO/MDI data together with SOHO/EIT $304 \AA$ (dominated by transition-region emission at $\sim 50,000 \mathrm{~K}$ ) and $195 \AA$ images covering a time series of a bit more than 3 days of observations taken in March 2006. The analysis represents a pixel light-curve distribution diagnostics that includes the width of the intensity distribution divided by the mean intensity in the pixel, distribution skewness, and distribution kurtosis. From the intensity variations at the location of the CBPs detected in the $304 \AA$ channel and the fact that some CBPs may exist only at transition region temperatures (i.e., do not reach coronal temperatures), the authors proposed that BPs (at TR and coronal temperatures) are subject to a two-stage heating process. The first involves reconnection driven by magneto-convection at the junctions of supergranular cells producing a BP at transition-region temperatures (named cool BPs). The second stage would start after the BP that is energetically stronger rises and expands in the solar corona and separator reconnection initiates between the expanding loops of the CBP and the overlying corona (e.g., Longcope et al. 2001) that leads to the CBP plasma being heated to coronal temperatures (producing a hot BP). In their study on the cool and hot components of a CBP, Tian et al. (2008a) speculate based on the analysis of magnetic field evolution and topology derived with the MPOLE code (for details see Sect. 4.2) that the formation and evolution of this CBP conform with the two-stage heating process suggested by McIntosh (2007).

In summary, CBPs are associated with photospheric flux of opposite polarities called magnetic bipoles/dipoles. In some cases the photospheric field is more complex than a simple bipole resulting in complicated loop connectivities as seen in coronal imaging channels. While $50 \%$ of CBPs in the quiet Sun are related to bipolar flux emergence, the rest result from the chance encounter of pre-existing magnetic flux. The range of the total magnetic flux associated with CBPs has been poorly investigated with the first and last study dating back to 1977. Later, only average or single CBP values were reported. While the upper boundary of the magnetic flux is believed to be $\sim 10^{20} \mathrm{Mx}$, it remains an open question what the minimum magnetic flux required to produce a small-scale loop with enhanced coronal emission (a hot loop) is. It is also still to be investigated how the latitudinal distribution and the variation of the number density of CBPs that form flux emergence and chance encounter of magnetic flux change during the 11-year activity cycle.

\subsection{Magnetic topology}

Knowledge about the three dimensional magnetic topology of CBPs is crucial in modelling these phenomena. Parnell et al. (1994a) were the first to derive the magnetic topology of two CBPs. They used a few photospheric point sources to reproduce the $3 \mathrm{D}$ structure of the magnetic field. The CBP structure was found to be aligned with the separator line connecting two 3D null points located in the photosphere. Pérez-Suárez et al. (2008) used the MPOLE topology code of Longcope (1996) to obtain the 3D structure of a CBP observed with several instruments on board Hinode and SoHO, as well as TRACE. The MPOLE code reduces a magnetogram to a distribution of monopoles in the photosphere to derive the $3 \mathrm{D}$ potential magnetic field. The comparison of the 
processed (using edge detection function) Hinode/XRT images and the extrapolated field (from SOHO/MDI magnetograms) revealed a very close agreement between the calculated magnetic field configuration and some of the X-ray loops composing the studied CBP (Fig. 17). The authors concluded that a large fraction of the magnetic field in the CBP is close to potential. By using the same methodology Alexander et al. (2011) reconstructed the topology of a single CBP reaching the same conclusion as Pérez-Suárez et al. (2008). None of the above studies investigated the configuration of the magnetic field overlying the CBP loop structure.

Zhang et al. (2012) employed a potential field model to derive the magnetic topology of 13 CBPs using longitudinal magnetic field data archived by the Global Oscillation Network Group (GONG) and obtained by the BBSO and Learmonth observatories. Two out of the 13 QS CBPs were associated with the so-called "embedded bipolar region" where one polarity magnetic-flux fragment is surrounded by opposite polarity concentrations. This photospheric magnetic field organization usually results in a coronal magnetic null point defining a dome-shaped separatrix surface. The remaining 11 CBPs were found to be associated with simple bipolar regions creating loop systems, also found by Pérez-Suárez et al. (2008) and Alexander et al. (2011).

Most recently Galsgaard et al. (2017) applied a potential model to reconstruct the magnetic skeleton of ten CBPs using Hinode/SOT and SDO/HMI magnetic field, and SDO/AIA and X-ray Hinode/XRT imaging data. In contrast to Zhang et al. (2012) they found that in nine CBPs the presence of one polarity flux surrounded by opposite polarity flux in a coronal hole and QS defines a magnetic null point above the minority flux. In these nine cases, the CBPs registered in X-rays or EUV is located in a limited part of the projected fan-dome area, either fully inside the dome or expanding over a limited dome area where typically dominant flux concentrations reside (Fig. 18). The tenth bright point occupies a simple bipolar loop system without an overlying null point. The authors also found that the magnetogram resolution does not play a role in the definition of the dome configuration. The paper discusses the possible reason for the differences with the study by Zhang et al. (2012).

\section{Plasma properties}

Knowledge about the physical parameters of the plasma such as flows (obtained either as proper motions of emission/absorption features in imaging data or from Doppler shifts in spectroscopic observations), electron density, temperature, filling factor etc. is fundamentally important for establishing the driving physical mechanism(s) of solar phenomena. Verification of all or some of these physical parameters that matches model derived parameters is the most reliable approach to constrain or determine the possible physical mechanism(s) at work. In the following the plasma properties of CBPs obtained both from imaging (monochromatic and passband) and EUV spectroscopic data are reviewed. 
Fig. 17 Left panels: SOHO/MDI magnetograms with overplotted MPOLE extrapolated coronal magnetic field. Right panels: edge-detection processed Hinode/XRT images. Image reproduced with permission from Pérez-Suárez et al. (2008), copyright by ESO

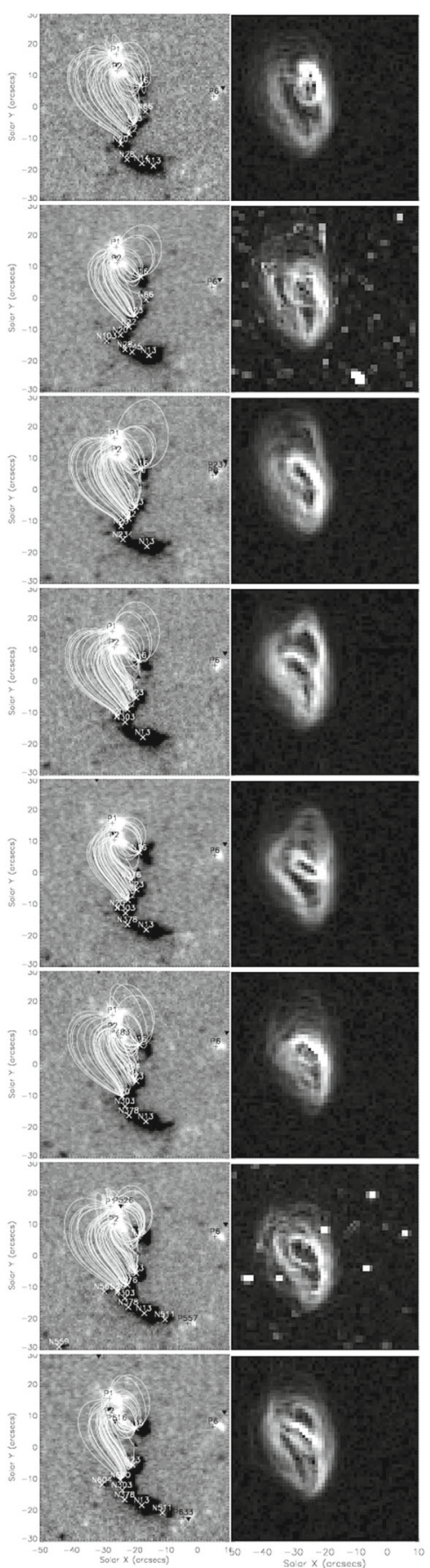



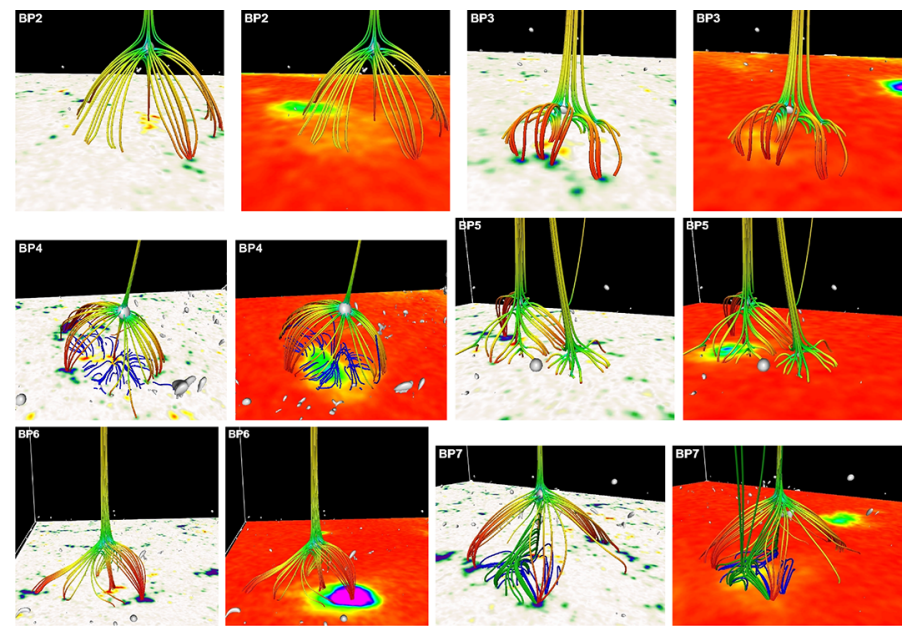

Fig. 18 Magnetic coronal structure of CBPs from potential field extrapolations. Image reproduced with permission from Galsgaard et al. (2017), copyright by ESO

\subsection{CBPs in EUV and X-rays emission}

One of most characteristic properties of CBPs is the apparent spatial and temporal variability of their emission across all EUV and X-ray wavelengths, as well as prominent radio signal variations (see Sect. 5.2). The first observations of CBPs in EUV spectral lines formed at chromospheric, transition-region and coronal temperatures revealed that the EUV emission from CBPs varies dynamically on timescales as short as the data cadence (Habbal and Withbroe 1981). Strong emission enhancements in coronal lines were accompanied by a strong intensity increase in chromospheric and transition region lines. Spectroheliograms were taken with the Harvard EUV experiment on Skylab/ATM at a $5^{\prime \prime}$ spatial resolution and 5.5 min cadence in the C II $1335 \AA$, Ly- $\alpha 1216 \AA$, O IV $554 \AA$, O VI $1032 \AA$, C III $977 \AA$, and Mg X $625 \AA$ lines. The observations obtained close to the solar limb showed that the coronal emission in CBPs extends a few arcseconds above their chromospheric emission conforming with the idea that CBPs are composed of magnetic loops rooted in the chromosphere. The coronal emission increase was found to precede the lower temperature emission (Fig. 19) suggesting that the heating occurs at coronal heights and is carried to the chromosphere by thermal conduction that can explain both the response in the chromospheric lines (e.g., $\mathrm{Ly}-\alpha$ ) and the initial response at coronal temperatures (e.g., $\mathrm{Mg} \mathrm{X}$ ).

Spectroheliograms from the Harvard EUV experiment on Skylab/ATM were used by Habbal et al. (1990) to compare some of the properties of CBPs in coronal holes and the quiet Sun including their morphological structure and emission variations. Simultaneous observations in six different spectral lines formed in the chromosphere, transition region and corona were used. The short-time variations in spectral lines with different formation temperatures (e.g., $\mathrm{MgX}$ and $\mathrm{C}$ III) were not always found to be co-spatial which may suggest that CBPs are composed of loops of various sizes and temperatures. Temporal variability of the emission at all wavelengths was observed 
Fig. 19 CBP intensity variations in different EUV spectral lines including $\mathrm{Mg} \mathrm{X}, \mathrm{O}$ VI, $\mathrm{C}$ II and Ly- $\alpha$. Image reproduced with permission from Habbal and Withbroe (1981), copyright by Springer

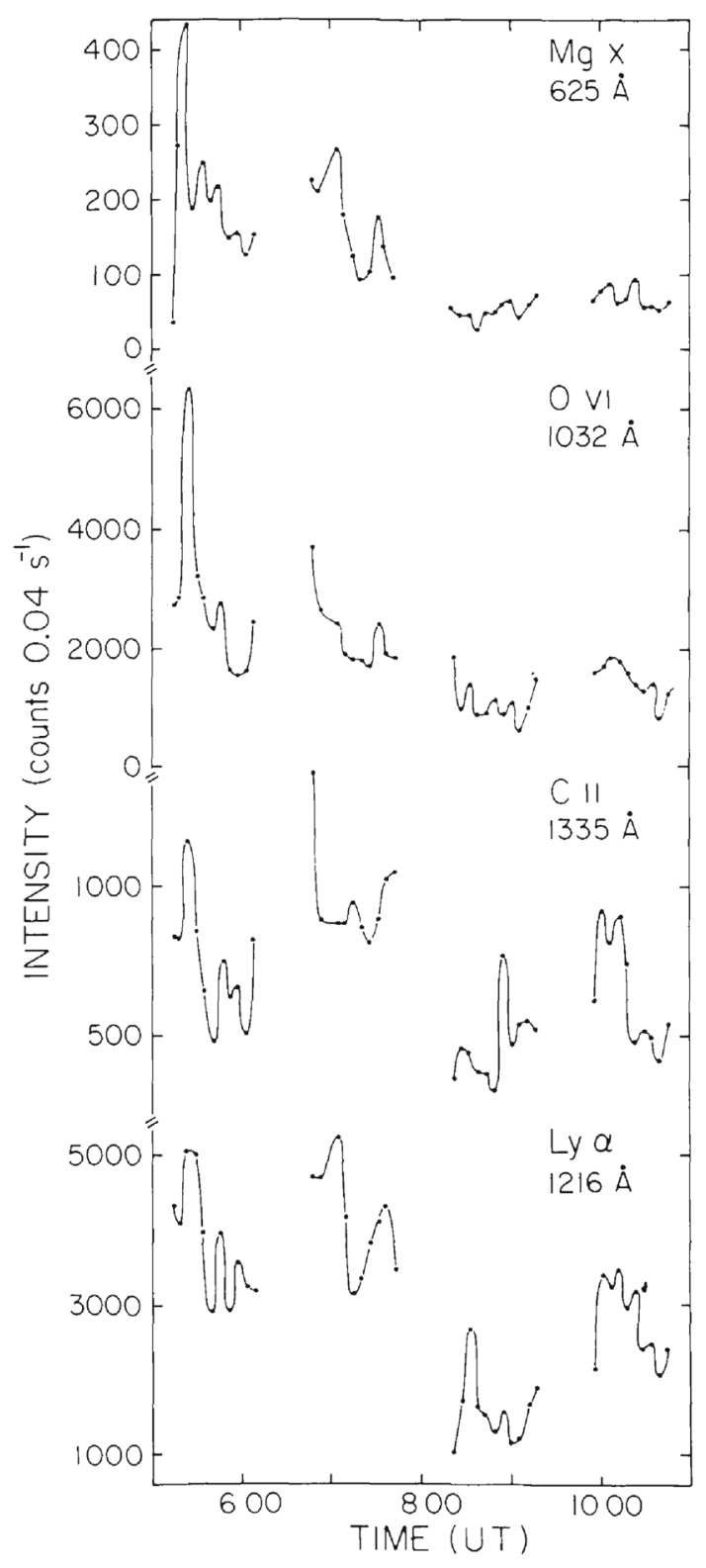

in CBPs from the two topologically different regions. The study also established that more CBPs are present at transition region temperatures than at coronal, both in coronal holes and in the quiet Sun. The intensity variability suggests that the CBP heating is eventually maintained by two heating mechanisms, a steady and a superimposed impulsive. The authors found two distinctive peaks of the parameter that is produced by the ratio of the varying (ac) and constant (dc) components of the emission (for details see their Sect. III, b), one at $\log T(\mathrm{~K}) \sim 5.0-5.3$ that is the peak of the radiative 
loss function, and a second for some CBPs at the $\mathrm{MgX}$ formation temperature of $\log T(\mathrm{~K}) \sim 6.1$ that corresponds to the conductive losses. The two peaks are found to vary significantly from CBP to CBP with a correlation between the change in the magnitude of ac/dc peaks and the mean intensity of the CBP. The emission variations were attributed to heating by magnetic reconnection that consequently would produce fast MHD waves which in the plasma and magnetic field environment of the CBPs would also contribute to the heating of the CBP plasma. The study concludes that the characteristic properties of CBPs in coronal holes and quiet Sun are independent of the background large-scale magnetic structure.

The emission variability in the quiet Sun has been largely attributed to CBPs and has been found to have a strong temperature dependence. Habbal and Grace (1991) studied EUV data (from the Harvard EUV experiment on Skylab/ATM) in six different spectral lines, Ly- $\alpha$, C II, C III, O IV, O VI and MgX at a 5" spatial and 5.5 min time resolution. Both the quiet Sun and coronal hole regions were investigated to eliminate the discrepancies related to the background large-scale magnetic configuration. A clear temperature dependence was found in the spatial distribution of the enhanced and variable emission, with a minimum at $\log T(\mathrm{~K}) \sim 5.47$, which divides two preferential distributions of the closed magnetic structures in the quiet Sun: one that forms at transition region temperatures, below $\log T(\mathrm{~K}) \sim 5.47$, and one at coronal. This implies that depending on the associated magnetic field strength, some CBPs could be composed of loops that do not reach coronal temperatures (see in Sect. 3.6 more on the CBP occurrence rate at different temperatures). The enhanced emission was found to cover only $10-25 \%$ of the solar surface depending on the temperature, while only $5-15 \%$ is caused by the variable emission. It has also been established that radiative losses from the variable emission are up to $40 \%$ from those of the enhanced emission. However, it is concluded that these losses are much smaller than the amount of heating required for maintaining the coronal temperature of the quiet Sun. The study also found that the ratio of the total radiative losses in the quiet Sun and coronal holes is the same implying that the energy input that causes the variable emission is independent of the general coronal magnetic structure.

Orange et al. (2014) compared a bright point observed at transition-region temperatures and a transient intensity brightening named blinker (Harrison 1997) concluding that "the blinker phenomena and the TR BP are sufficiently dissimilar in their observed properties as to constitute different event classes." The term 'blinker' refers to transient phenomena detected at transition-region temperatures that show intensity enhancements of a factor of 2-3. Blinkers have average lifetimes ranging from 6 to $40 \mathrm{~min}$ and typical sizes of $3 \times 10^{7} \mathrm{~km}^{2}$ (Harrison 1997; Bewsher et al. 2002). Harrison et al. (2003) unified under the term blinker all events named as blinkers, network and cell brightenings, and EUV brightenings that have been identified in SOHO/CDS and EIT data and concluded that they represent the "same phenomenon detected using different techniques". Most recently Subramanian et al. (2012) using multi-instrumental co-observations concluded that actually "blinkers are the EUV response of various transient events originating at coronal, transition region and chromospheric heights". Some of these phenomena were EUV/X-ray jets, brightenings in small-scale loops (i.e., a CBP) or foot-point brightenings of larger loops. Therefore, some blinkers can eventually be linked to CBPs. 


\subsection{CBPs at radio wavelengths}

Interferometric observations ranging from 0.8 to $11 \mathrm{~cm}$ and a spatial resolution from $4^{\prime \prime}$ to $2^{\prime}$ of the quiet Sun have revealed already in 1970s surprisingly strong interferometric signals varying at time scales from 5 to $30 \mathrm{~min}$ (e.g., Zirin et al. 1978; Janssen et al. 1979, and the references therein). Initially, this was interpreted as related to the chromospheric network. The opportunity for detailed investigations of the origin of these interferometer signals was presented when the VLA radio observatory became operational.

Quiet Sun solar observations at a frequency of $4.9 \mathrm{GHz}(6 \mathrm{~cm})$ made with the VLA during the partial solar eclipse on 1977 October 12 showed that at small spatial scales the observations are dominated by a small number of compact sources (Marsh et al. 1980). The sources' sizes had a range of $9^{\prime \prime}-25^{\prime \prime}$ and a peak brightness temperature of $6-8 \times 10^{4} \mathrm{~K}$ with respect to the background temperature of $2 \times 10^{5} \mathrm{~K}$, with an absolute brightness temperature of the strongest source of $1.0 \times 10^{5} \mathrm{~K}$. At least three out of six sources were associated with bipolar magnetic features strongly suggesting that small microwave sources represent CBPs. The authors found no associations with the chromospheric network and went to suggest that the quiet Sun radio signal is mainly produced by CBPs. An earlier study by Hachenberg et al. (1978) has already associated numerous bipolar magnetic regions in the quiet Sun with radio sources at $8.5 \mathrm{~mm}$ wavelength that had lifetimes of several hours and showed intensity variations at times scales of a few minutes.

Habbal et al. (1986) reported the first observations of CBPs at $20 \mathrm{~cm}$ wavelength with VLA at $15^{\prime \prime}$ spatial resolution. Three radio sources were identified in the quiet Sun and were associated with dark points in He I 10,830 ̊ which are known to be the counterpart of CBPs. The sources had a size of $\sim 40^{\prime \prime}$ and a brightness temperature ranging from $5 \times 10^{4}$ to $5 \times 10^{5} \mathrm{~K}$, the temperature that characterizes the transition region. Under the assumption of free-free emission, the observed variations (Fig. 20) of the brightness temperature in the range between 1 and $5 \times 10^{5} \mathrm{~K}$ were found to be caused by pressure fluctuations at transition region heights combined with "either plasma motions along magnetic field lines or changes in the magnetic field topology, or both". The magnetic field strength was deduced for the first and only time from the brightness temperature of the extraordinary and the ordinary wave modes and it was found to vary between 50 and $200 \mathrm{G}$.

Further observations of CBPs at $6 \mathrm{~cm}$ wavelength taken with the VLA at a spatial resolution as high as $1.2^{\prime \prime}$ (Fu et al. 1987) revealed that the radio emission associated with CBPs that show rapid temporal and spatial variations is similar to the variations observed in X-rays, EUV, optical wavelengths, and at $20 \mathrm{~cm}$ wavelength. The spatial variations appeared either as irregular displacements or structural changes. The sources had a diameter of $5^{\prime \prime}-15^{\prime \prime}$ and lifetime of 5-20 min which was believed to be related to the lifetime of the small-scale loops that form CBPs. The brightness temperature (above the quiet Sun background) had a range between $0.2 \times 10^{4}$ and $3 \times 10^{5} \mathrm{~K}$. It is speculated that the $6 \mathrm{~cm}$ sources are located near the footpoints of these loops and the small-duration sources are related to flaring in CBPs. The maximum brightness temperature of the sources was estimated at $\sim 3 \times 10^{4} \mathrm{~K}$ with an average value of 


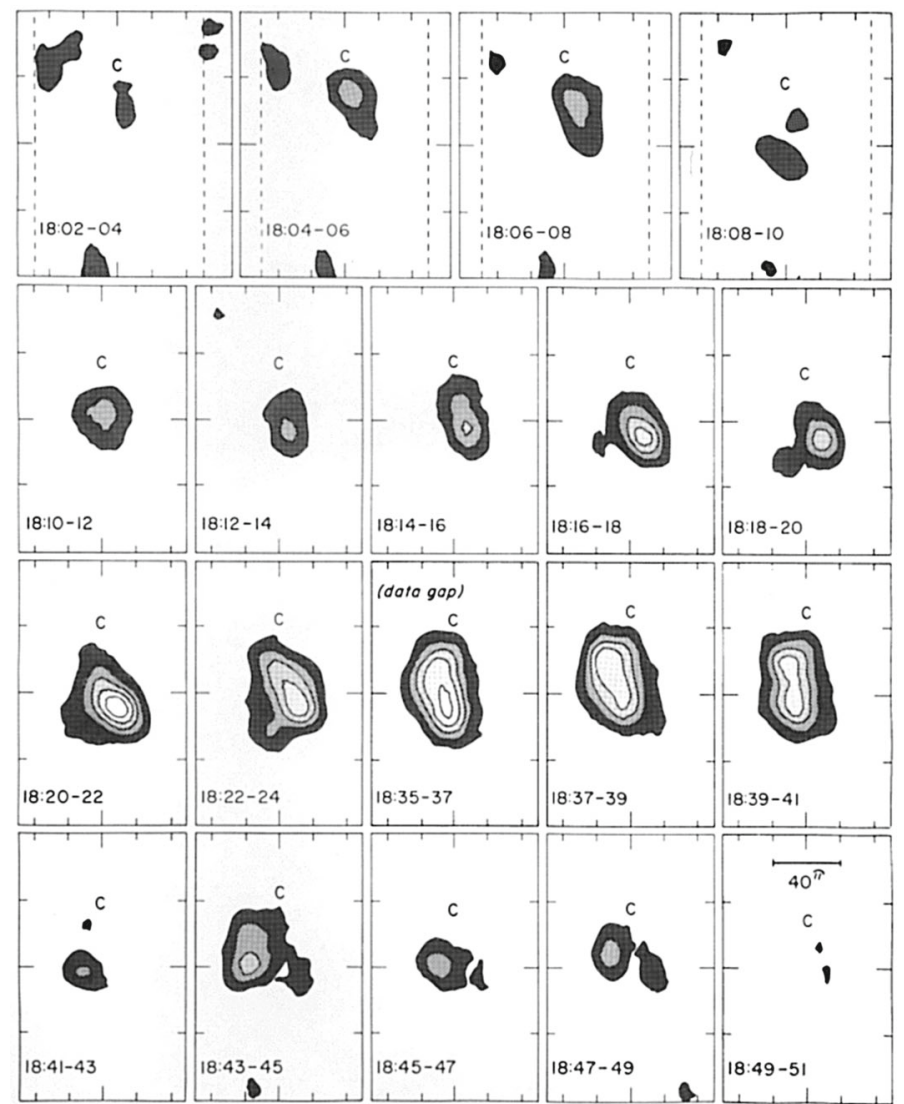

Fig. 20 Intensity maps at $\lambda=20 \mathrm{~cm}$ of two bright points and contour levels that are multiples of $5 \times 10^{4} \mathrm{~K}$, the $2.5 \sigma$ level. Image reproduced with permission from Habbal et al. (1986), copyright by AAS

$\sim 1 \times 10^{4} \mathrm{~K}$. From the difference between the distances from the centre of the solar disk to the radio sources and to co-temporally observed dark points in He I 10,830 $\AA$, the height of the $6 \mathrm{~cm}$ CBP sources was estimated at $1.5-2.0 \times 10^{4} \mathrm{~km}$ above the He I formation height that is however far above the footpoints of the CBP loops.

More studies at radio wavelengths by Kundu et al. (1988), Habbal and Harvey (1988), and Nitta and Kundu (1988) followed. Kundu et al. (1988) analysed VLA data in the radio 6 and $20 \mathrm{~cm}$, and estimated their average brightness temperature ratio of 0.09 which conforms with thermal bremsstrahlung emission. Habbal and Harvey (1988) reported the first simultaneous observations of a quiet Sun region in He I $10,830 \AA$ and at the radio $20 \mathrm{~cm}$ wavelength, combined with magnetic field data. The radio emission was found always to coincide with the He I emission but not always with dark points, i.e., CBPs (see Fig. 21). Only 30-35\% of the radio mission was associated with strong or moderate He I dark points, while both the radio emission and the He I absorption showed rapid intensity variations (as low as the time resolution of $3 \mathrm{~min}$ ). These variations were not found to be co-temporal and were often associated 
Fig. 21 Lightcurves of the emission of the radio $20 \mathrm{~cm}$ (top panels) and He I absorption (bottom panels) sources. Filled circles denote the co-temporal data points in both wavelengths. Open circles are data that exist only in one dataset. Top-left panel shows the flux density of the radio emission $\left(I\left(J_{y}\right)\right)$, while the right top panel gives the maximum brightness temperature $T_{B}$ of the source. The bottom-left panel presents the mean intensity $(|\bar{I}|)$ of the He I absorption and the bottom right the maximum $\left(\left|I_{\max }\right|\right)$. Image reproduced with permission from Habbal and Harvey (1988), copyright by AAS
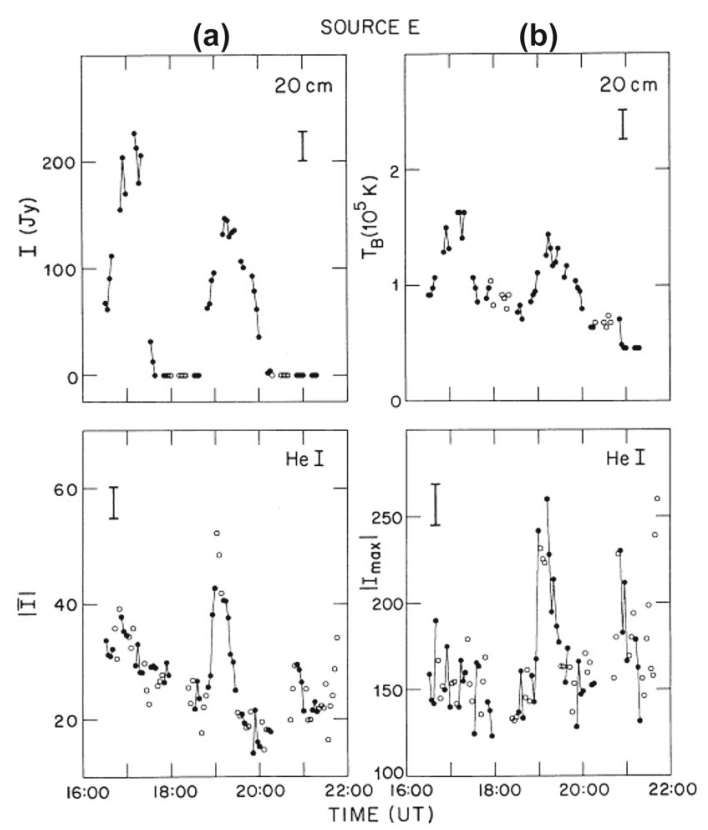

with spatial variations of the $20 \mathrm{~cm}$ sources. Most importantly magnetic cancelation rather than magnetic flux emergence were found to relate to these intensity fluctuations.

Nitta et al. (1992) compared CBPs observed in soft X-rays by Yohkoh/SXT with radio $20 \mathrm{~cm}$ observations. Half of the 33 sources were associated with XBPs, while the rest were overlaying unipolar regions. Therefore, CBPs are not the solely source of radio emission and cannot be used as an identifier of this phenomenon. The derived CBP temperatures of 1.4-2.9 $\times 10^{6} \mathrm{~K}$ and emission measure of $0.4-$ $2.5 \times 10^{45} \mathrm{~cm}^{-3}$, with a brightness temperature of $1-2.5 \times 10^{5} \mathrm{~K}$, suggested optically thin bremsstrahlung emission. Kundu et al. (1994a) also reported a $17 \mathrm{GHz}$ radio emission from CBPs (four cases) in the QS and AR. Corresponding XBPs were simultaneously detected by Yohkoh/SXT. Two XBPs showed flaring activity while the other two quiescent intensity fluctuations. The flaring CBP gradual, long-lasting $(\sim 2 \mathrm{~h})$, unpolarized emission, with a radio peak almost simultaneous as the X-ray intensity peak, indicated that this $17 \mathrm{GHz}$ emission is thermal in nature. Using the $\mathrm{X}$-ray emission and a filter ratio method, the temperature, density and emission measure were derived to estimate the thermal emission at $17 \mathrm{GHz}$. The calculated values were found to be larger than the observed ones which is assumed to be caused by the emission from lower temperature plasma not detectable by Yohkoh/SXT but sensitive to microwave free-free emission. A contribution from non-thermal electrons should also be considered.

Non-thermal particle acceleration is a clear indication of a large energy release (e.g., in the course of magnetic reconnection) during which free magnetic energy is converted to kinetic energy, usually observed in the time of solar flares in active regions. Flaring CBPs were associated with radio type III bursts during the Skylab mission (Kundu 
et al. 1980), as well as later in the Yohkoh era (Kundu et al. 1994b, 1995). Kundu et al. (1980) analysed $430 \mathrm{~h}$ of data from the Clark Lake Radio Observatory interferometer and found 4 radio burst events from 29 flaring CBPs registered by Skylab, i.e., only a $10 \%$ association. Kundu et al. (1994b) studied Nançay Radioheliograph (NRH) metric radio emission and co-temporal soft X-ray observations of CBPs by Yohkoh/SXT and established that flares in XBPs located in coronal holes give rise to non-thermal emission in the form of type III radio bursts. Kundu et al. (1995) provided further evidence for radio type III bursts at the time of XBP microflarings and also reported on the temporal evolution of the emission. The soft X-ray intensity variations that showed an increase from 100 to $700 \%$ (i.e., microflaring, see Sect. 7.1) during tens of minutes were well correlated with the radio type III burst groups. The radio bursts occurred during the impulsive phase of the XBP microflares and were located close or at the XBPs (Fig. 22). These findings indicated that CBP flares have a similar origin to AR flares and also suggested that the physical mechanism that takes place can produce non-thermal electron beams. As radio III bursts require open magnetic field (e.g., coronal hole fields), this phenomenon is expected to be observed less frequently than the microwave or radio emission for instance, which could explain the low association rate.

To summarise, radio sources at 6 and $20 \mathrm{~cm}$ wavelength that are believed to be produced by optically thin free-free bremsstrahlung emission have been associated with CBPs. Brightness temperature variations were suggested to be associated pressure fluctuations caused by plasma flows along field lines or changes of the magnetic topology at transition region heights. The magnetic field strength was deduced for the first and only time from the brightness temperature of the extraordinary and the ordinary wave modes and it was found to vary between 50 and 200 G. Magnetic cancelation rather than magnetic flux emergence were found to relate to these intensity fluctuations of the radio sources. Flaring CBPs were associated with radio type III bursts.

\subsection{Doppler and non-thermal velocities}

Plasma flows in CBPs (non-eruptive quiet phase of their evolution) have only been determined from Doppler shift measurements given their small sizes and the limitation of the pixel size of the existing imaging data with respect to these sizes (mainly corona - $0.6^{\prime \prime}$ pixel size of SDO/AIA, and transition region- $0.16^{\prime \prime}$ for IRIS). Doppler velocities in CBPs are reported from data taken by several spectrometers including the High Resolution Telescope and Spectrograph (HRTS), the SUMER/SoHO spectrometer, Hinode/EIS, IRIS and EUNIS rocket mission. Spectroscopic observations in the C IV $1548 \AA$ line recorded by HRTS were used to investigate the relation of XBPs and explosive events (transition region emission showing strong non-Gaussian profiles with Doppler shifts of up to $250 \mathrm{~km} \mathrm{~s}^{-1}$ ) (Moses et al. 1994). No relation was found between explosive events and XBPs, and Doppler widths in the range of $20-40 \mathrm{~km} \mathrm{~s}^{-1}$ in XBPs were derived. Vilhu et al. (2002) reported a spectroscopic study from a flaring CBP in SUMER observations. The data used in this study represent 44 scans made with a $2^{\prime \prime}$ slit. The resulting images had a field-of-view of $23^{\prime \prime} \times 24^{\prime \prime}$ and 2.8 min cadence. 

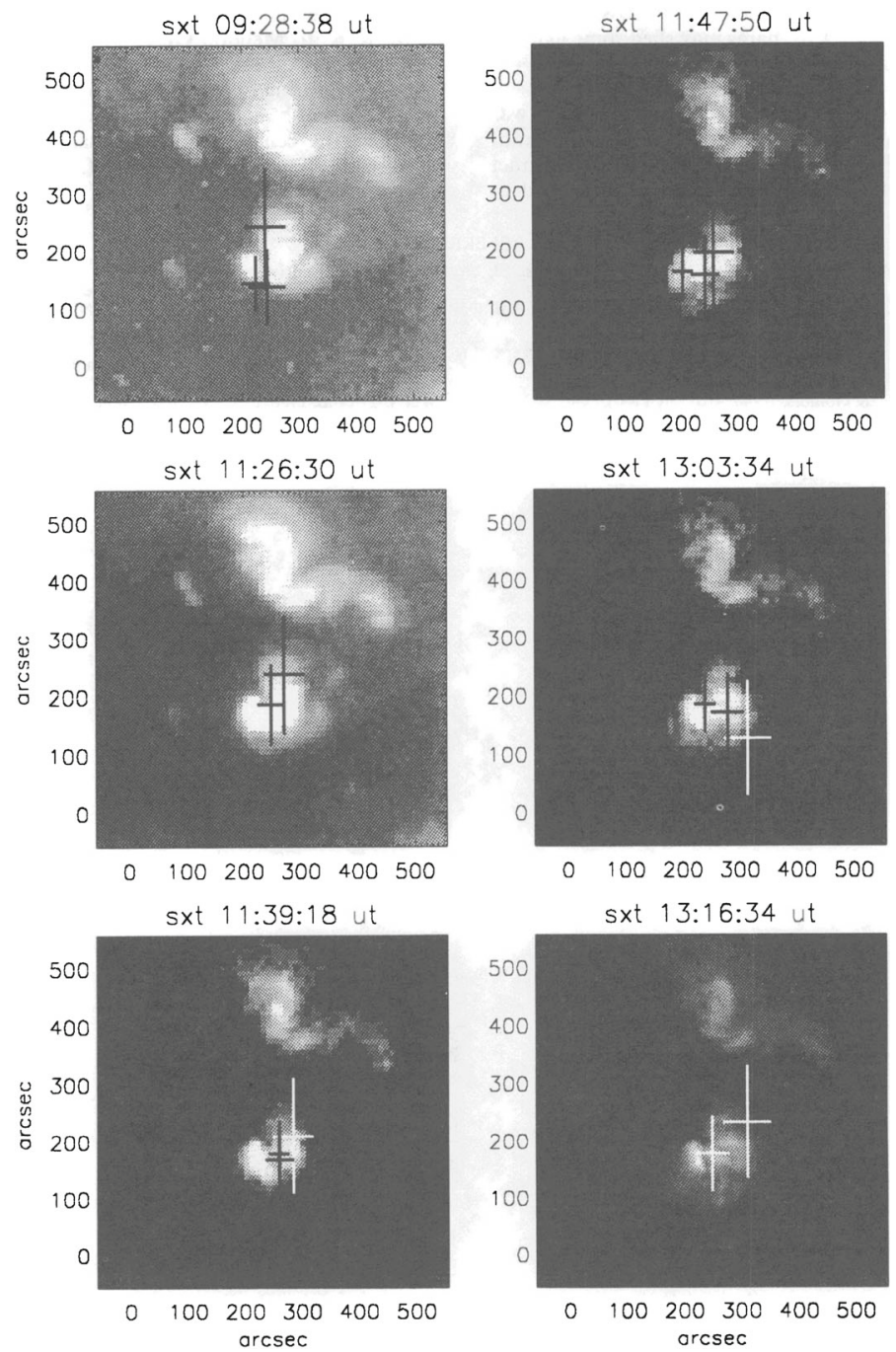

Fig. 22 Locations of the metric type III radio bursts superimposed on the Yohkoh/SXT images. Image reproduced with permission from Kundu et al. (1995), copyright by AAS

High average non-thermal velocities in the $\mathrm{CBP}$ were obtained in the transition region N II $775.96 \AA\left(T_{\max } \approx 2.5 \times 10^{4} \mathrm{~K}\right)$, N IV $765.15 \AA\left(T_{\max } \approx 1.26 \times 10^{5} \mathrm{~K}\right)$, O IV $787.74 \AA\left(T_{\max } \approx 1.6 \times 10^{5} \mathrm{~K}\right)$, and coronal Ne VIII $770.42 \AA\left(T_{\max } \approx 6.3 \times 10^{5} \mathrm{~K}\right)$ lines of $40 \pm 10 \mathrm{~km} \mathrm{~s}^{-1}, 44 \pm 5 \mathrm{~km} \mathrm{~s}^{-1}, 46 \pm 5 \mathrm{~km} \mathrm{~s}^{-1}$, and $41 \pm 5 \mathrm{~km} \mathrm{~s}^{-1}$, respectively. The formation temperature $T_{\max }$ refers to the temperature at the maximum ionization fraction and it will be indicated henceforward for each mentioned spectral line. The nature of the non-thermal velocity is still largely open to discussion and it is believed to be a manifestation of small-scale laminar flows, waves and/or turbulence present in the observed plasma at a certain temperature. For optically thin lines the 
non-thermal velocity is obtained from the measured full width at half maximum after a correction for the instrumental broadening and is given by the expression

$$
F W H M=\left[4 \ln 2\left(\frac{\lambda}{c}\right)^{2}\left(\frac{2 k_{B} T_{i}}{M}+\xi^{2}\right)\right]^{1 / 2},
$$

where $k_{B}$ is the Boltzmann constant, $M$ is the mass of the atom emitting the line, $T_{i}$ is the ion temperature assumed to be equal to the electron temperature, and $\xi$ is the non-thermal velocity.

By combining potential and linear force-free field extrapolations using line-of-sight magnetograms from the Kitt Peak National Observatory and spectroscopic observations in the Si IV $1402 \AA$ line taken with the ultraviolet spectrometer and polarimeter (UVSP) on the Solar Maximum Mission (SMM) (Woodgate et al. 1980), Rovira et al. (1999) derived red-shifted emission at both ends of the nearly potential magnetic loops that are consistent with downflows in both loop legs. An enhanced X-ray emission at $8 \times 10^{6} \mathrm{~K}$ lead the authors to the suggestion that forced reconnection due to continuous flux emergence is the cause of the CBP heating. The flow pattern would be consistent with chromospheric evaporation due to coronal magnetic reconnection between the emerging and overlying coronal field.

Using SUMER observations, Madjarska et al. (2003) derived Doppler shifts in a QS CBP in the S VI $933.40 \AA\left(2.0 \times 10^{5} \mathrm{~K}\right)$ line ranging from -10 to $10 \mathrm{~km} \mathrm{~s}^{-1}$. The data were taken in a sit-and-stare mode (rastering with SUMER was restricted for instrumental reasons) and no rotational compensation was applied, resulting in the slit "scanning" $10^{\prime \prime}$ in solar longitude (at the targeted heliographic coordinates) in one hour. The area above the bipole magnetic flux concentrations was found to have red-shifted emission, while blue-shifted emission was recorded in the centre of the CBP. Therefore, the transition region emission is produced in the legs/footpoints of the CBP loops rooted in the bipole flux, while the blue-shifted signal comes from the background corona (in the present case, a coronal hole) rather than coming from the CBP loop top at coronal heights. This red-shifted emission could be interpreted as produced by downflows resulting from cooling plasma that drains towards the photosphere. The physical processes leading to this are still to be investigated. Smallscale brightenings in the CBP were found to have velocities in the range of 3-6 km s${ }^{-1}$. Also in a transition region line registered with SUMER, O III $703.87 \AA\left(8.0 \times 10^{4} \mathrm{~K}\right)$, Popescu et al. (2004) reports a red-shifted emission at $5 \mathrm{~km} \mathrm{~s}^{-1}$ from a narrow cut through the outer edge of a CBP, above one of the footpoints, and $0 \mathrm{~km} \mathrm{~s}^{-1}$ in the centre of the CBP. In contrast, the emission in the coronal Mg IX $706.02 \AA$ line $\left(1.0 \times 10^{6} \mathrm{~K}\right)$ is $\sim-4 \mathrm{~km} \mathrm{~s}^{-1}$ (the CBP is located in a polar coronal hole). Orange et al. (2014) obtained Doppler shifts of $\leq 25 \mathrm{~km} \mathrm{~s}^{-1}$ in a CBP registered in transition region spectral lines obtained with Hinode/EIS, and $\leq 20 \mathrm{~km} \mathrm{~s}^{-1}$ in a CBP seen in coronal lines except for the case when microflaring and jet was observed.

Most recently, Kayshap and Dwivedi (2017) analysed two CBPs observed with IRIS located in a coronal hole. Similarly to Madjarska et al. (2003), they found in the two legs/footpoints of the BP loops redshifted emission in the Si IV $1393.75 \AA\left(8.0 \times 10^{4} \mathrm{~K}\right)$ in the range of $5-30 \mathrm{~km} \mathrm{~s}^{-1}$ (see Fig. 23), $\sim 5-20 \mathrm{~km} \mathrm{~s}^{-1}$ in the CII $1394.76 \AA$ 

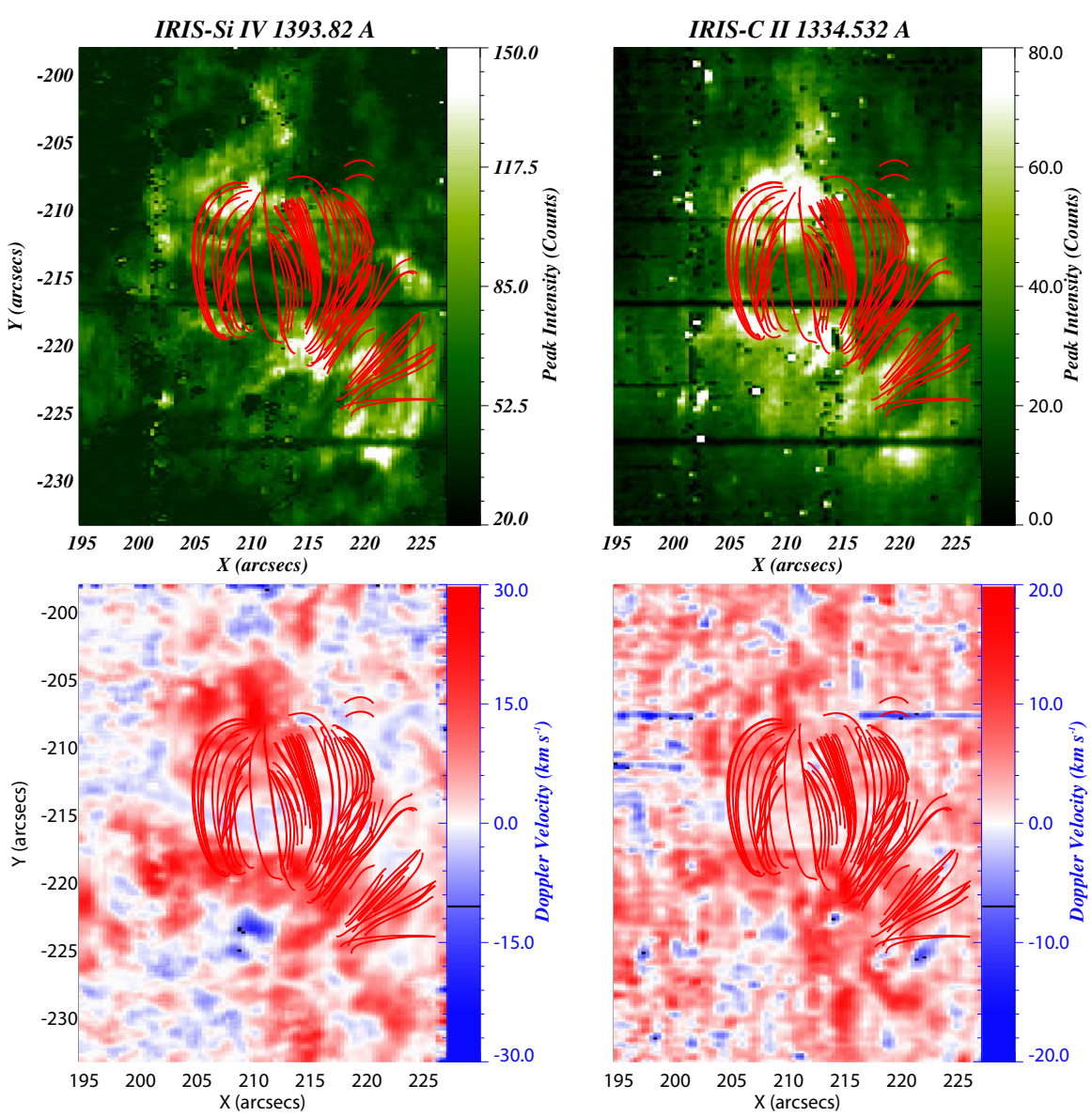

Fig. 23 Intensity (top panel) and Doppler velocity (bottom panel) in SiIV (left column) and C II (right column). The red lines represent the magnetic field lines derived from a potential field extrapolation using SDO/HMI longitudinal magnetograms. Images reproduced with permission from Kayshap and Dwivedi (2017), copyright by Springer

$\left(2.5 \times 10^{4} \mathrm{~K}\right.$, see Fig. 23$)$, and $\sim 10 \mathrm{~km} \mathrm{~s}^{-1}$ in the OIV $1401.16 \AA\left(1.6 \times 10^{5} \mathrm{~K}\right)$. Blue-shifted emission is recorded in the centre of the CBP which is emitted from the $\mathrm{CH}$ background.

The EUNIS sounding rocket instrument provided spectroscopic data that were used by Brosius et al. (2007) to derive Doppler shifts in CBPs in spectral lines covering a large temperature range (Fig. 24). The Doppler velocities obtained as a function of the position across the CBP (solar x positions) in five strong spectral lines are $\pm 15 \mathrm{~km} \mathrm{~s}^{-1}$ in He II $\left(5.0 \times 10^{4} \mathrm{~K}\right), \pm 14 \mathrm{~km} \mathrm{~s}^{-1}$ in Mg IX $368.1 \AA\left(9.5 \times 10^{5} \mathrm{~K}\right), \pm 26 \mathrm{~km} \mathrm{~s}^{-1}$ in Fe XIV $334.2 \AA\left(2.0 \times 10^{6} \mathrm{~K}\right)$, and $\pm 35 \mathrm{~km} \mathrm{~s}^{-1}$ in both Fe XVI 335.4 and $360.8 \AA$ $\left(2.5 \times 10^{6} \mathrm{~K}\right)$, the highest temperature line used in Doppler shift measurements of CBPs). The authors concluded that the same Doppler shift pattern is observed in all four spectral lines: blue-shifted on the eastern side and red-shifted on the western side of the CBP (the footpoint/bipole orientation is north-south). The CBP is located in a 

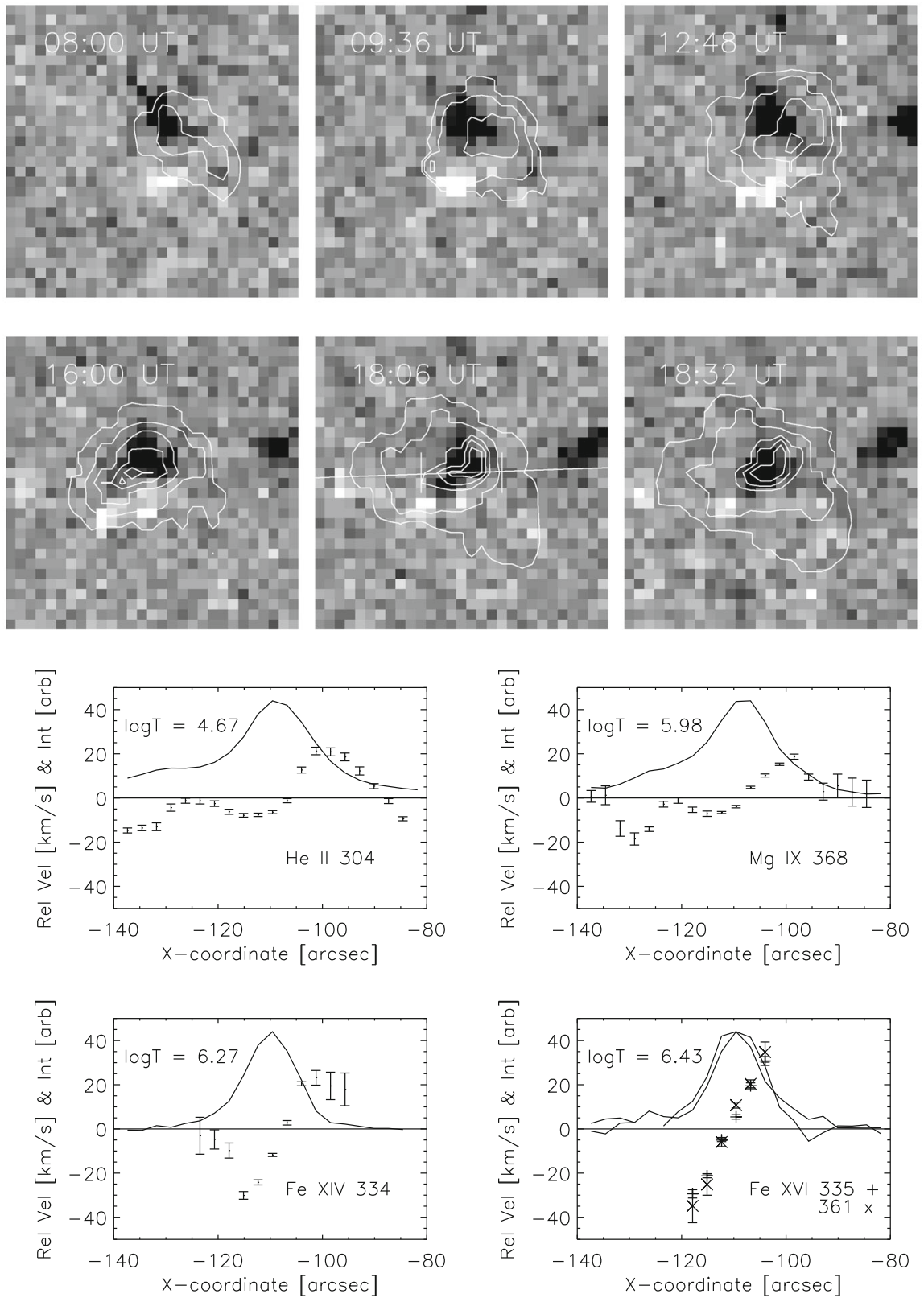

Fig. 24 Top two rows: SOHO/MDI longitudinal magnetograms scaled to $\pm 50 \mathrm{G}$ overlaid with the SOHO/EIT $195 \AA$ A contours. The horizontal line represents the EUNIS slit. The field of view of the images is $56.5^{\prime \prime} \times 56.5^{\prime \prime}$. Bottom two rows: intensity (solid line) and Doppler velocity including error bars along the EUNIS slit in six different spectral lines as noted on the figure panels. Images reproduced with permission from Brosius et al. (2007), copyright by AAS 

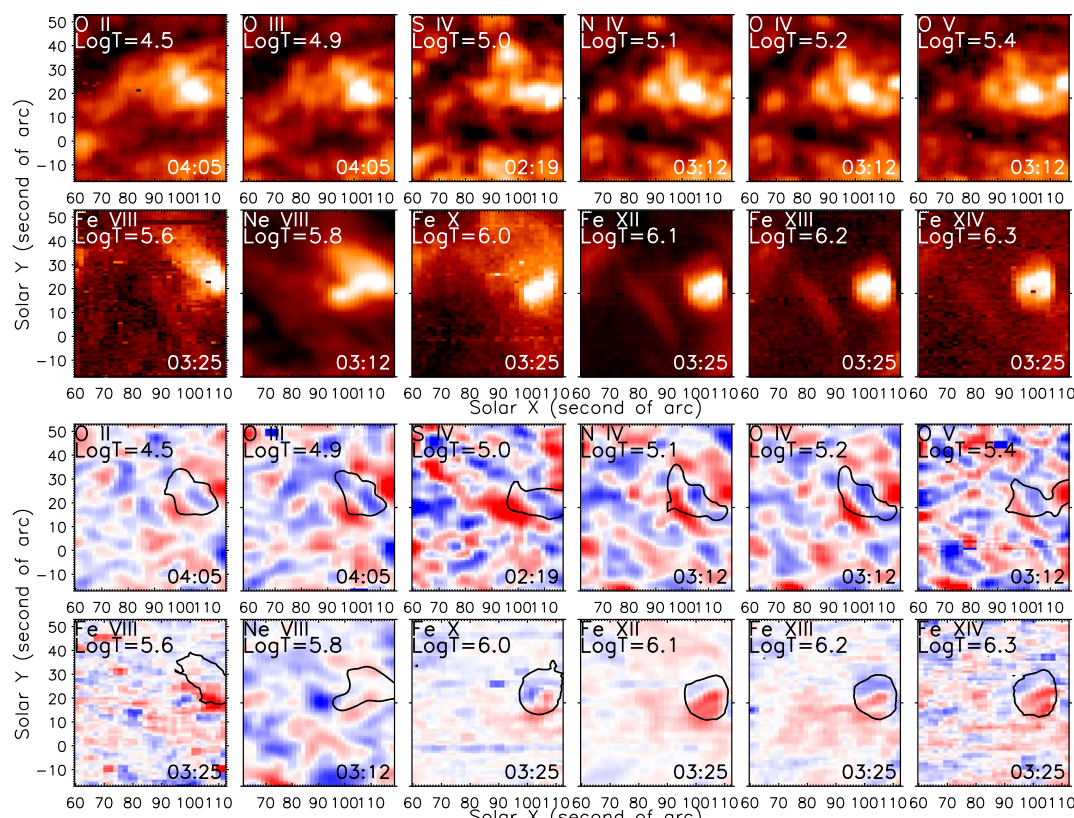

6070809010011060708090100110
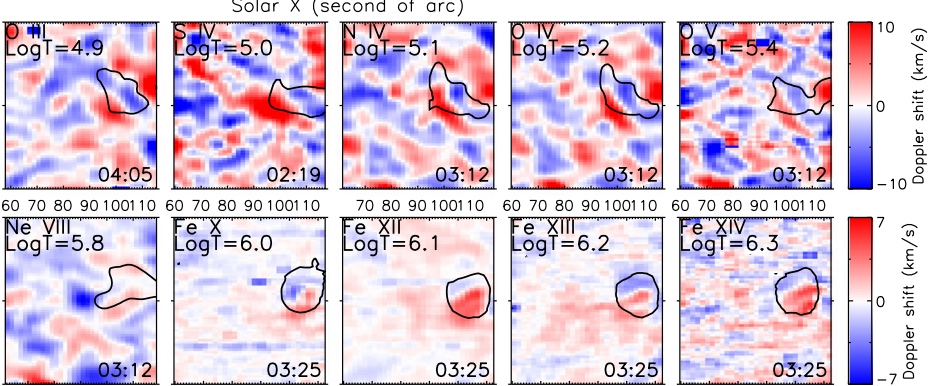

60708090100110

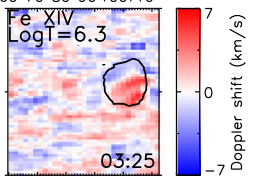

Fig. 25 Top two rows: intensity images of a CBP in transition region (first row) and coronal lines (second). Bottom two rows: Doppler shift images in the spectral lines shown in the top two rows. The count outlines the position of the CBP in each spectral line. The time in the right corner indicates approximately the scanning time. Images reproduced with permission from Tian et al. (2008a), copyright by AAS

polar coronal hole that needs to be taken into consideration while interpreting those Doppler velocities. In the study the slit crosses the CBP in a fixed single position across the CBP loops and therefore does not provide information about the Doppler shifts in the legs/footpoints of the CBP loops as in the study by Madjarska et al. (2003). However, from the provided images it is evident that the slit crosses the brightest top of the CBPs (see the contour lines overplotted on each panel in Fig. 24). Thus, at the position of the peak intensity, the emission from the coronal lines Mg IX 368.1 $\AA$, Fe XIV $334.2 \AA$, and Fe XVI $335.4 \AA$ is blue-shifted to up to $15 \mathrm{~km} \mathrm{~s}^{-1}$.

Pérez-Suárez et al. (2008) used Hinode/EIS data and obtained Doppler shifts in six spectral lines with different formation temperatures. The obtained Doppler velocities are in the range $\pm 15 \mathrm{~km} \mathrm{~s}^{-1}$ in Si VII $275.35 \AA\left(6.3 \times 10^{5} \mathrm{~K}\right), \mathrm{Fe} \times 184.54 \AA(1.0 \times$ $\left.10^{6} \mathrm{~K}\right) - \pm 10 \mathrm{~km} \mathrm{~s}^{-1}$, Fe XII $195.12 \AA\left(1.3 \times 10^{6} \mathrm{~K}\right)- \pm 8 \mathrm{~km} \mathrm{~s}^{-1}$, from 6 to $18 \mathrm{~km} \mathrm{~s}^{-1}$ in Fe XIII $202.04 \AA\left(1.6 \times 10^{6} \mathrm{~K}\right)$, and $\pm 15 \mathrm{~km} \mathrm{~s}^{-1}$ in Fe XIV $270.52 \AA\left(2.0 \times 10^{6} \mathrm{~K}\right)$. Using both SUMER/SoHO and also Hinode/EIS data Tian et al. (2008a) obtained different Doppler shift values in spectral lines formed in the transition region (from SOHO/SUMER) and corona (from Hinode/EIS and SOHO/SUMER Ne VIII), naming them cool and hot CBP components. The Doppler-shift range in the transition region lines was found to be slightly higher than in the coronal lines (Fig. 25). The 'cool' and 'hot' two components of the CBP present a blue-red shifted pattern with a very different orientation which the authors interpreted as resulting from a twist of the magnetic loop system. 
Also from SOHO/SUMER data, Xia et al. (2003) reports blue-shifted emission in the range $3-7 \mathrm{~km} \mathrm{~s}^{-1}$ in Ne VIII $770.42 \AA$. Doppler-shift images were obtained assuming a net blue shift in the Ne VIII of $-5 \mathrm{~km} \mathrm{~s}^{-1}$ (value derived when the line is observed in second order permitting then to use $\mathrm{C}$ I lines for wavelength calibration). Tian et al. (2008a) obtained Doppler shifts close to zero in the same line. On the other hand, Tian et al. (2009) obtained blue-shifted emission of up to $9.5 \mathrm{~km} \mathrm{~s}^{-1}$ in both legs of CBP loops by combining magnetic field extrapolation using a potential field model and spectroscopic observations in the SOHO/SUMER Ne VIII $770.42 \AA$ line. These flows were interpreted as the product of continuous reconnection between internetwork small scale loops that continuously emerge and are swept via the supergranular convection to the network boundaries, where they eventually interact with larger loops or open field lines named funnels (see the cartoon shown in Fig. 4 of their paper). This would produce upflows in upper-transition region and low-coronal lines, and downflows in lower temperature (with respect to Ne VIII) transition region lines (e.g., S VI in Madjarska et al. 2003 and Si IV observations in Kayshap and Dwivedi 2017).

Plasma flows in CBPs are an important indicator of the physical processes that are at work to produce both the steady heating of CBPs plasma or impulsive energy release that triggers eruptions including jets and mass ejections. The wide range of values from the several studies mentioned above do not reveal a fully consistent picture either because of line-of-sight, instrumental and/or wavelength calibration effects. Nevertheless, one SOHO/SUMER (Madjarska et al. 2003) and one IRIS study (Kayshap and Dwivedi 2017) of CBPs located away from polar and limb regions clearly show red-shifted emission in transition-region spectral lines from the CBP legs/footpoints. The emission in coronal lines CBP loop tops indicate blue-shifted emission in CBPs (Xia et al. 2003; Popescu et al. 2004; Brosius et al. 2007) with stronger shifts in spectral lines with higher formation temperatures. It remains paramount that plasma flows from Doppler shift measurements in CBPs are further investigated in data from present and/or past missions to provide important constrains to the modelling of these phenomena.

Various values of Doppler velocities were derived in CBPs using spectroscopic data from several spectrometers. Disk centre observations that are most suitable for Doppler shift measurements indicate that the low transition-region emission in CBPs is red-shifted while high transition-region and coronal lines show blue-shifted emission that may be consistent with footpoint heating. Some studies also reveal syphon flows (upflow in one leg and downflow in the other). Further Doppler velocity measurements in CBPs in spectral lines with a large range of formation temperatures are required. A precise as possible rest-wavelength calibration, removal of the effects caused by distorted point-spread function, and other instrumental effects are also important to be taken into account.

\subsection{Electron densities}

The electron pressure $\left(N_{e} T_{e}\right)$ is an important plasma parameter in theoretical modelling and therefore reliable information about the plasma electron densities and temperatures is fundamentally important. The electron density is often deduced from imaging data 
that assumes knowledge of the emitting plasma volume and homogenous plasma distribution within. However, the solar plasma is confined in filamentary structures that present observations cannot spatially resolve and thus the most accurate method to estimate the electron density is from the ratio of spectral lines of the same ion that makes no assumption about the size of the observed plasma volume or the elemental abundance values.

CBP electron densities were first obtained from data taken with SOHO/CDS as part of a polar plume study, Del Zanna et al. (2003) derived the electron density of a single CBP using line ratios of O IV $625.9 \AA$ and $608.9 \AA$ (hereafter the ratios are denoted as 625.9/608.9), Mg VII 319.0/367.7, and Si IX 349.9/341.9, and obtained $N_{e}$ of $1.9 \pm 0.4 \times 10^{10} \mathrm{~cm}^{-3}, 0.9 \pm 0.5 \times 10^{9} \mathrm{~cm}^{-3}$, and $5.0 \pm 2 \times 10^{8} \mathrm{~cm}^{-3}$, respectively. Ugarte-Urra et al. (2004a) obtained a similar value from the Si IX 349.9/341.9 line ratio of $5.0 \times 10^{8} \mathrm{~cm}^{-3}$.

A specially dedicated study on the electron densities of CBPs by Ugarte-Urra et al. (2005) estimated $N_{e}$ in six CBPs observed by CDS/SoHO in spectral lines with formation temperatures ranging between 1.3 and $2.0 \times 10^{6} \mathrm{~K}$. The average $N_{e}$ values from the line ratios Fe XIV 353.79/334.17, Fe XIII 359.78/348.18, Fe XII 364.47/338.26, and Si X 356.04/347.40 derived without the background subtraction are $2.14 \times 10^{9} \mathrm{~cm}^{-3}, 4.6 \times 10^{9} \mathrm{~cm}^{-3}, 3.3 \times 10^{9} \mathrm{~cm}^{-3}$ and $9.1 \times 10^{9} \mathrm{~cm}^{-3}$, respectively. For more information on spectral line blends and the Chianti database versions used by the different studies, the respective articles should be consulted. Evidently, the CBP plasma densities are more similar to active regions than to those of the quiet Sun.

Brosius et al. (2008) used EUNIS data to derive $N_{e}$ in a CBP. The FeXI $308.52 / 352.67$ line ratio gives an electron density $N_{e}=2.6 \times 10^{9} \mathrm{~cm}^{-3}$, Fe XII $338.26 / 346.87-4.9 \times 10^{9} \mathrm{~cm}^{-3}$, Fe XIII 320.82/348.18-3.5 $\times 10^{9} \mathrm{~cm}^{-3}$, Fe XIV $353.58 / 334.17-3.3 \times 10^{9} \mathrm{~cm}^{-3}$, and for $\mathrm{Si} \times 356.02 / 347.41-2.5 \times 10^{9} \mathrm{~cm}^{-3}$ (formation temperature range $\log T_{\max }(\mathrm{K})$ from 6.1 to 6.3$)$. The line pair with the lowest formation temperature $\mathrm{Mg}$ VII yields $N_{e} 1.0 \times 10^{9} \mathrm{~cm}^{-3}\left[\log T_{\max }(\mathrm{K}) \sim 5.8\right]$. Tian et al. (2008a) obtained similar values at coronal temperatures $\left[\log T_{\max }(\mathrm{K})\right.$ from 6.1 to 6.3] in the range 1.2-3.2 $\times 10^{9} \mathrm{~cm}^{-3}$ (see Fig. 26) as Del Zanna et al. (2003), Brosius et al. (2007), and Ugarte-Urra et al. (2005) using Hinode/EIS data. They also derived $N_{e}$ from the transition region line ratios of O IV 1407.39/1401.16 and OV 753.68/761.13 (SOHO/SUMER data) of $\sim 4.0 \times 10^{10} \mathrm{~cm}^{-3}$ and $\sim 1.3 \times 10^{10} \mathrm{~cm}^{-3}$, respectively, values that again are closer to AR densities. Several other studies with Hinode/EIS data give $N_{e}$ at $4 \times 10^{9} \mathrm{~cm}^{-3}$ (average value in Fe XII, Dere 2008), $3.7 \times 10^{9} \mathrm{~cm}^{-3}$ in Fe XII and Fe XIII (Pérez-Suárez et al. 2008), and $3 \times 10^{9} \mathrm{~cm}^{-3}$ (median value from several CBPs in FeXII, Dere 2009). Doschek et al. (2010) studied several CBPs in coronal holes and derived electron densities in Fe XII, Fe XIII and Fe XIV in the range $0.5-1 \times 10^{9} \mathrm{~cm}^{-3}$, lower than the densities found by previous authors. It should be mentioned here that $N_{e}$ are typically higher when obtained in Fe XII than in Fe XIII that is believed to be related to uncertainties in the atomic data related to the Fe XII lines. The most recent study by Schmelz et al. (2013) reports $N_{e}$ in 12 CBPs from Hinode/EIS Fe XII 186.88/195.12 line ratios in the range $6-9 \times 10^{8} \mathrm{~cm}^{-3}$ that are also significantly lower than the values obtained earlier from this line pair. The author of the present review assumes that the authors that have used EIS data have taken into 
Fig. 26 Top panel: intensity ratios as a function of the electron density of seven spectral line pairs. Bottom panel: electron densities of a CBP obtained from the line pairs shown in the top panel. Image reproduced with permission from Tian et al. (2008a), copyright by AAS
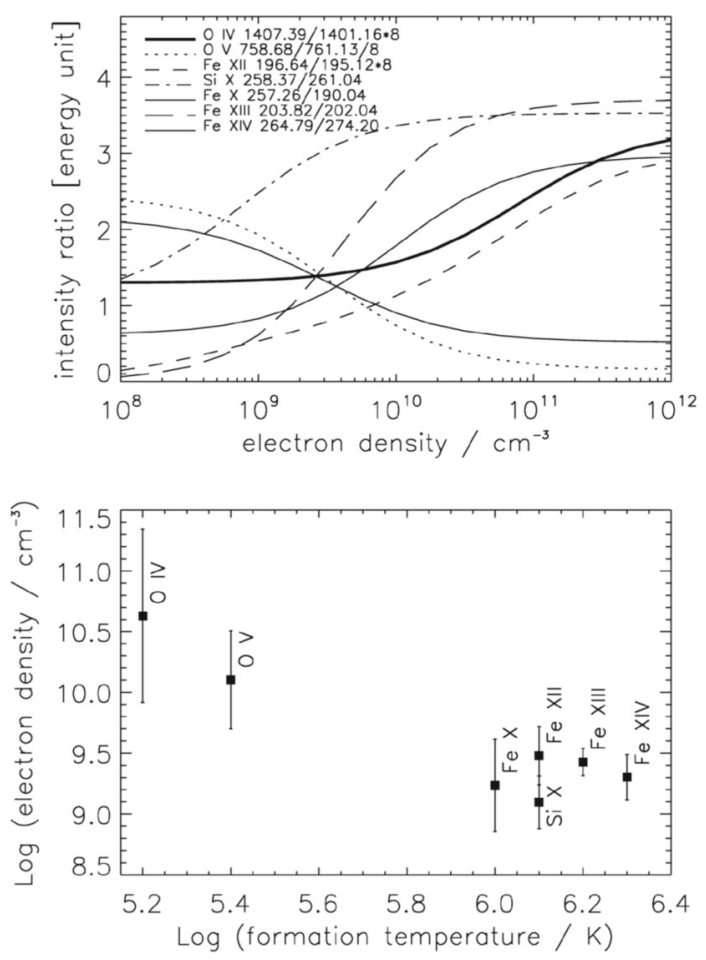

account the blends by Fe XII $186.89 \AA$ and $195.18 \AA$, as it is not mentioned in some of the studies.

Alexander et al. (2011) also studied the $N_{e}$ of a quiet-Sun CBP from the Fe XII $($ Hinode/EIS) $(186.85+186.89) /(195.12+195.18)$ line ratio in data taken over a time period of $\sim 13 \mathrm{~h}$ (22 Hinode/EIS rasters). This long Hinode/EIS time series permitted to derive the electron density evolution of the CBP which appeared quite steady at $\sim 5.0 \times 10^{9} \mathrm{~cm}^{-3}$, but decreased by $\sim 40 \%$ in the last hour (raster) of the observations. The temporal changes of the electron density were also studied by Ugarte-Urra et al. (2004a). In the transition region, the ratio of O IV 625.8/608.4 shows a small variation of the $N_{e}$ around an average value of $3 \times 10^{10} \mathrm{~cm}^{-3}$. It should be noted that the obtained $N_{e}$ does not cover the full lifetime of the CBP as in Alexander et al. (2011). Kamio et al. (2011) studied the plasma properties of microflares in CH and QS CBPs using the Hinode/EIS Fe XII $(186.85+186.89) /(195.12+195.18)$ line ratio. A significant increase of the electron density in CBPs is found during a microflare that coincides with the peak of the Fe XII intensity for both CH and QS CBPs (see Fig. 27). An overview of the electron densities obtained until present is given in Table 1. The wavelength of some of the lines were changed from the original papers to unify them with other reports and with the CHIANTI database (done by the author of the present review).

To conclude, the electron densities of CBPs show large similarities to AR densities with typically a few $10^{10} \mathrm{~cm}^{-3}$ at transition-region temperatures, and a few $10^{9} \mathrm{~cm}^{-3}$ 

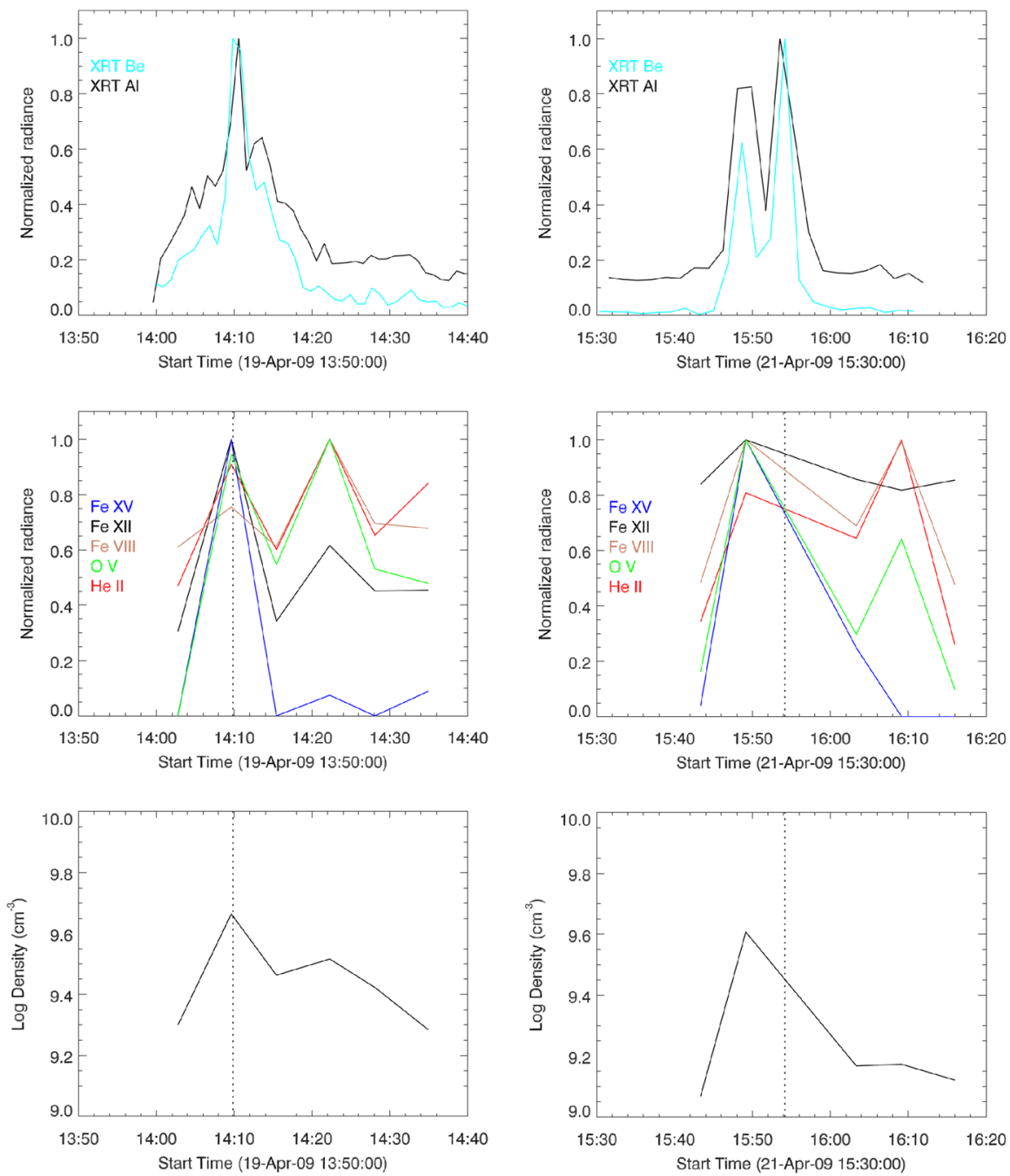

Fig. 27 Normalized lightcurves of a microflare in a QS CBP (left hand panels) and CH CBP (right hand panels). Top: X-ray lightcurves in two filters, Be_thin and Al_poly. Middle: lightcurves from Hinode/EIS emission lines. Bottom: the electron density $\left(\mathrm{cm}^{-3}\right)$ form Fe XII 186.85/195.12. Image reproduced with permission from Kamio et al. (2011), copyright by ESO

at coronal, clearly indicating that CBPs represent downscaled active regions. Coronal densities remain relatively constant during CBP lifetimes. More than 3 times density increase is found during microflaring in CBPs.

\subsection{Temperatures}

Several methods have been used to determine the temperatures of CBPs including the two filter image ratios, the emission measure loci (EM-loci) method and spectral 
Table 1 Electron densities of CBPs

\begin{tabular}{|c|c|c|c|c|c|}
\hline Ion & Line ratio $(\AA / \AA)$ & $\begin{array}{l}\log T_{\max } \\
(\log K)\end{array}$ & $\begin{array}{l}\text { Electron density } \\
\left(\mathrm{cm}^{-3}\right)\end{array}$ & Instrument & References \\
\hline OIV & $1407.39 / 1401.16$ & 5.2 & $4.0 \times 10^{10}$ & SUMER & 5 \\
\hline OIV & $625.90 / 608.40$ & 5.2 & $1.9 \times 10^{10}$ & CDS & 1 \\
\hline OIV & $625.90 / 608.40$ & 5.2 & $3.0 \times 10^{10}$ & CDS & 3 \\
\hline $\mathrm{OV}$ & $753.68 / 761.13$ & 5.4 & $1.3 \times 10^{10}$ & SUMER & 5 \\
\hline Mg VII & $319.03 / 367.70$ & 5.8 & $0.9 \times 10^{9}$ & CDS & 1 \\
\hline Mg VII & $319.03 / 365.22$ & 5.8 & $1.0 \times 10^{9}$ & EUNIS & 3 \\
\hline $\mathrm{Fex}$ & $257.26 / 190.04$ & 6.1 & $1.6 \times 10^{9}$ & EIS & 5 \\
\hline Si IX & $349.90 / 341.90$ & 6.1 & $5.0 \times 10^{8}$ & CDS & 1,2 \\
\hline \multirow[t]{2}{*}{$\operatorname{Six}$} & \multirow[t]{2}{*}{$356.04 / 347.40$} & \multirow[t]{2}{*}{6.1} & $9.1 \times 10^{9}$ & CDS & 3 \\
\hline & & & $2.5 \times 10^{9}$ & EUNIS & 4 \\
\hline $\operatorname{Six}$ & $258.37 / 261.04$ & 6.1 & $1.3 \times 10^{9}$ & EIS & 5 \\
\hline $\mathrm{FeXI}$ & $308.52 / 352.67$ & 6.1 & $2.6 \times 10^{9}$ & EUNIS & 4 \\
\hline Fe XII & $364.47 / 338.26$ & 6.1 & $3.3 \times 10^{9}$ & CDS & 3 \\
\hline FeXII & $338.26 / 346.87$ & 6.1 & $4.9 \times 10^{9}$ & EUNIS & 4 \\
\hline \multirow[t]{7}{*}{ FeXII } & \multirow[t]{7}{*}{$186.85 / 195.12$} & \multirow[t]{7}{*}{6.1} & $4.0 \times 10^{9}$ & EIS & 6 \\
\hline & & & $3.7 \times 10^{9}$ & EIS & 7 \\
\hline & & & $3.0 \times 10^{9}$ & EIS & 4,8 \\
\hline & & & $8.9 \times 10^{8}$ & EIS & 9 \\
\hline & & & $5.0 \times 10^{9}$ & EIS & 10 \\
\hline & & & $6-9 \times 10^{8}$ & EIS & 12 \\
\hline & & & $1-5 \times 10^{9}$ & EIS & 11 \\
\hline Fe XIII & $359.78 / 348.18$ & 6.2 & $4.6 \times 10^{9}$ & CDS & 3 \\
\hline Fe XIII & $320.82 / 348.18$ & 6.2 & $3.5 \times 10^{9}$ & EUNIS & 4 \\
\hline \multirow[t]{3}{*}{ Fe XIII } & \multirow[t]{3}{*}{$203.82 / 202.04$} & \multirow[t]{3}{*}{6.2} & $3.0 \times 10^{9}$ & EIS & 5 \\
\hline & & & $3.7 \times 10^{9}$ & EIS & 7 \\
\hline & & & $7.1 \times 10^{8}$ & EIS & 9 \\
\hline FexIV & $353.84 / 334.17$ & 6.3 & $2.1 \times 10^{9}$ & CDS & 3 \\
\hline FeXIV & $353.84 / 334.17$ & 6.3 & $3.3 \times 10^{9}$ & EUNIS & 4 \\
\hline \multirow[t]{2}{*}{ FexIV } & \multirow[t]{2}{*}{$264.79 / 274.20$} & \multirow[t]{2}{*}{6.3} & $2.0 \times 10^{9}$ & EIS & 5 \\
\hline & & & $1.1 \times 10^{9}$ & EIS & 9 \\
\hline
\end{tabular}

(1) Del Zanna et al. (2003), (2) Ugarte-Urra et al. (2004a), (3) Ugarte-Urra et al. (2005), (4) Brosius et al. (2008), (5) Tian et al. (2008a), (6) Dere (2008), (7) Pérez-Suárez et al. (2008), (8) Dere (2009), (9) Doschek et al. (2010), (10) Alexander et al. (2011), (11) Kamio et al. (2011), and (12) Schmelz et al. (2013)

observations. From the intensity ratio of CBPs observed with two X-ray filters (Be: 3$17 \AA$ and polypropylene: $3-32,43-54 \AA$ ) by the Skylab/S-054 X-ray telescope, Golub et al. (1974) estimated that temperatures of XBPs range between 1.3 and $1.7 \mathrm{MK}$ (an error of $50 \%$ is assumed) with an upper limit of $2 \mathrm{MK}$. This method implies that the observed plasma volume is strictly isothermal, which is not always valid for 


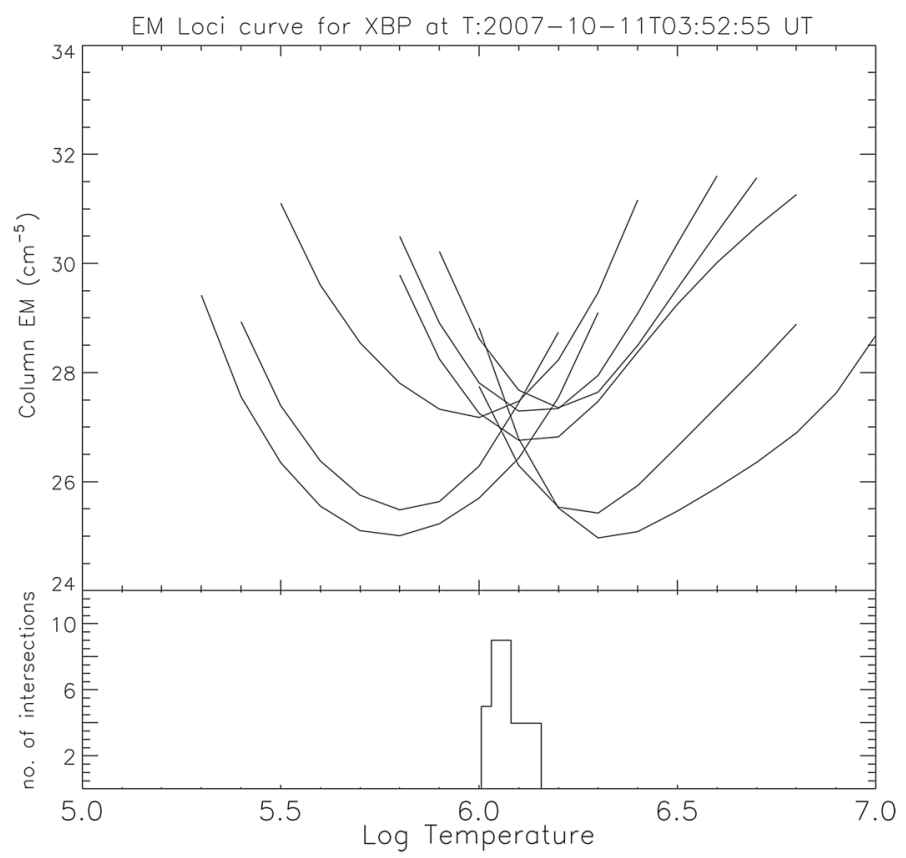

Fig. 28 Emission measure loci curves of a CBP (top panel) and number of intersections at different temperatures (bottom panel). Image reproduced with permission from Alexander et al. (2011), copyright by ESO

coronal structures. The method has been questioned as the filter wavelength bands cover spectral lines formed over a wide range of temperatures that contribute to the signal in each filter (e.g., Martens et al. 2002; Del Zanna and Mason 2003). The temporal variability of temperature was investigated by Kariyappa et al. (2011) using the Hinode/XRT Ti_poly and Al_mesh filter ratio. The temperatures of seven BPs were found to range from 1.11 to $2.31 \mathrm{MK}$ with one CBP reaching as high as $3.44 \mathrm{MK}$. The lightcurves of the intensity ratios revealed a high variability suggesting significant temperature variations.

Alexander et al. (2011) used the EM-loci method of Jordan et al. (1987) to estimate the temperature of a CBP registered in spectral (Hinode/EIS) and imaging (Hinode/XRT) data. The background-corrected emission in eight spectral lines from 22 scans of the CBPs taken during $\sim 13 \mathrm{~h}$ of Hinode/EIS observations was used. It was established that the CBP remained nearly isothermal during the observing period with temperatures in the range of 1.2-1.7 MK, with an average of 1.3 MK (see Fig. 28). Doschek et al. (2010) also used Hinode/EIS data to study several CBPs and concluded that CBPs reach temperatures not higher than 2-3 MK and have a multi-temperature structure with the hotter loops overlying the cooler ones. A multi-colour temperature presentation shown here in Fig. 29 supports this interpretation of the CBP temperature structure. Figure 10 of their paper presents the appearance of the CBP in spectral lines with different formation temperatures. The loop structure of the CBPs is not visible at transition region temperatures, but one can clearly see emission in these lines that 


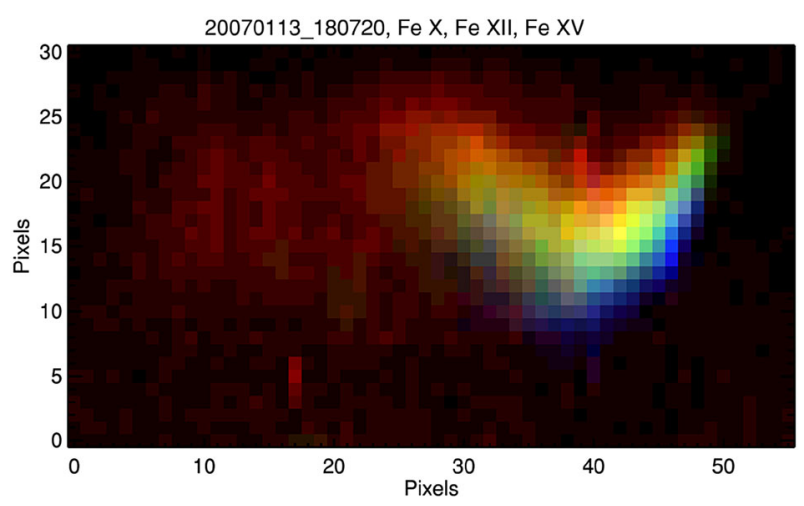

Fig. 29 CBP observed by Hinode/EIS on 2007 January 13. Blue colour marks the spectral line with the highest formation temperature, while red corresponds to the lowest. Image reproduced with permission from Doschek et al. (2010), copyright by AAS

is known to come from the footpoints of the CBP loops (e.g., Madjarska et al. 2003; Alexander et al. 2011). The EM-loci method was also used (see their Fig. 8) with an intersection of the emission measure lightcurves of FeX, FeXI, Fe XII and Fe XIII at 1.2 MK, except for the lightcurves of the highest ionization stage lines of Fe XIV and FexV.

The EM-loci method was also used by Orange et al. (2014) and the presence of single and double isothermal components in bright points are transition-region and coronal temperatures, respectively, were found. The broad distributions were interpreted as a signature of unresolved structures.

To summarise, CBPs are composed of loops at different temperatures ranging from 1.2 to as high as 3.4 MK, with hotter loops overlying cooler ones. The footpoints of CBP loops are clearly seen in chromospheric and transition region temperatures, while the plasma at loop tops produces emission predominantly at coronal temperatures.

\subsection{Plasma filling factor}

The volumetric plasma filling factor of CBPs was first estimated by Dere (2008) using Hinode/EIS data. The intensities of Fe XII $186.88 \AA$ and $195.12 \AA$ were used to derive the electron densities and the widths of 62 CBP loops. The widths were estimated as the full width of half maximum of the intensity profile perpendicular to the length of the loop, and were found to have a median value of $5^{\prime \prime}$ (30\% accuracy). CBPs in Hinode/EIS data often appear as a single loop (especially when scanned with a $2^{\prime \prime}$ slit) due to the resolution limitation. The volumetric filling factor $f$ of the Hinode/EIS CBPs was found to vary from $4 \times 10^{-5}$ to 0.2 with a median value of 0.015 using the expression:

$$
I=0.86 G\left(T_{\max }\right) N_{e}^{2} f w,
$$

where $G\left(T_{\max }\right)$ is the contribution function at its peak temperature $\left(T_{\max }\right)$ calculated with the Chianti database. Also the following assumptions are applied: $w$ is the loop width that represents the integration pathlength and for the formation temperature of 
Fe XII of $1.6 \times 10^{6} \mathrm{~K}$, the hydrogen density is $N_{H}=0.86 N_{e}$. The CBP was considered to be a cylindrical loop.

This filling factor range implies that the CBP loop resolved by Hinode/EIS should be composed of loops with median widths of $1^{\prime \prime}-2^{\prime \prime}$. The densities for a subset of the CBPs obtained with the Fe XIII were found to be twice lower than in Fe XII implying a four times lower filling factor, i.e., 0.06. The authors concluded that if CBPs are considered to be formed by subresolution (size below the resolving capabilities of Hinode/EIS) loops they would have a median width of $0.9^{\prime \prime}$ (when Fe XII electron densities are used) and 1.8" (at the Fe XIII densities).

To test these results, Dere (2009) later analysed TRACE data with electron densities derived from simultaneously taken Hinode/EIS data. TRACE has a factor of two higher spatial resolution compared to Hinode/EIS 1" slit raster data. The volumetric plasma filling factor was found to range from $3 \times 10^{-3}$ to 0.3 with a median value of 0.04 for a CBP with an average electron density of $3 \times 10^{9} \mathrm{~cm}^{-3}$ at a temperature of $1.6 \times 10^{6} \mathrm{~K}(\mathrm{Fe} \mathrm{XII})$. This result indicated that CBPs have a sub-EIS and even subTRACE resolution structure, and are composed by loops with widths in the range of $0.2^{\prime \prime}-1.5^{\prime \prime}$. Data at the spatial resolution of IRIS and the High-resolution Coronal Imager (known as Hi-C Imager, Cirtain et al. 2013) rocket flight are therefore required to further investigate this issue.

\subsection{Differential emission measure}

Brosius et al. (2008) first derived the differential emission measure (DEM) and elemental abundances (see the follow-up section) of a CBP using spectra taken with the EUNIS rocket mission. The DEM delivers important information on the temperature distribution of the plasma in the emitting source. It is related to both the electron density and to the temperature gradient of the plasma, and thus could provide important clues on the physical processes at work in the observed phenomenon. The EUNIS spectrum covers spectral lines with formation temperatures in the range of $\log T(\mathrm{~K}) \sim 5.2-6.4$. The obtained CBP DEM curve was found to have a single peak of log DEM $\sim 20.70$ at $\log T(\mathrm{~K}) \sim 6.15$ and a local minimum of $\log \mathrm{DEM} \sim 20.15$ at $\log T(\mathrm{~K}) \sim 5.35$ (see Fig. 30).

Doschek et al. (2010) obtained the DEM for two CBPs observed by Hinode/EIS using full CCD observations, i.e., all spectral lines that can be observed by the Hinode/EIS spectrometer, and have a high signal-to-noise ratio in CBPs. The CBPs were found to have DEMs with three clear peaks of $\log \mathrm{DEM} \sim 23.5$ at $\log T(\mathrm{~K}) \simeq 5.2$, $\log \mathrm{DEM} \sim 22.5$ at $\log T(\mathrm{~K}) \simeq 5.8$, and $\log \mathrm{DEM} \sim 23.5$ at $\log T(\mathrm{~K}) \simeq 6.15$ (see Fig. 31). The first peak is related to He II and OIV that are emitted in the legs/footpoints of the CBP loops and a $\operatorname{dip}$ at $\log T(\mathrm{~K}) \simeq 5.5$. The second peak at $\log T(\mathrm{~K}) \simeq 5.8$ is related to the background region of the coronal hole (see their Fig. 13). The highest peak at $\log T(\mathrm{~K}) \simeq 6.15$ describes the coronal temperature structure of the CBPs and is identical to the DEM peak in Brosius et al. (2008) who, however, misses the first peak due to the lack of transition-region temperature spectral lines in EUNIS. 


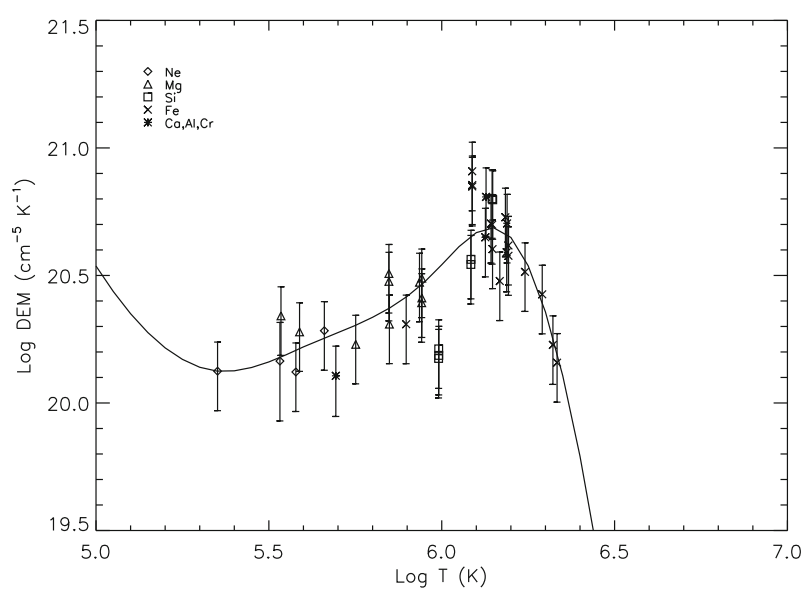

Fig. 30 DEM as a function of temperature for a CBP observed with EUNIS. Image reproduced with permission from Brosius et al. (2008), copyright by AAS
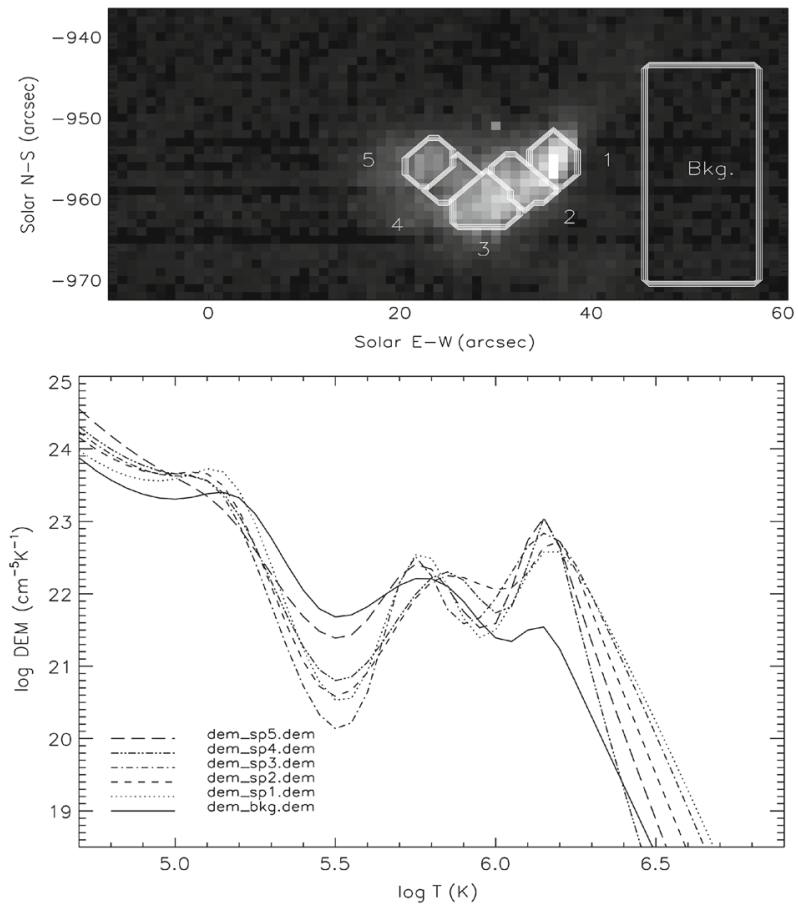

Fig. 31 Top panel: Fexv $284.16 \AA\left[\log T_{\max }(\mathrm{K}) \sim 6.3\right]$ intensity image. The outlined areas denoted the regions from which the average intensity is used to obtain the DEM curves shown in the bottom panel. Bottom panel: DEM curves as a function of temperature for the five regions of the CBP and the background region of the coronal hole. Images reproduced with permission from Doschek et al. (2010), copyright by AAS 
Imaging data were also used to derive DEMs and their time evolution for CBPs. Chitta et al. (2013) employed 6 SDO/AIA EUV channels—94 $\AA, 131 \AA, 171 \AA$, $193 \AA, 211 \AA$, and $335 \AA$ to produce DEMs that cover the temperature range of $\log T(\mathrm{~K}) \sim 5.6-6.5$. The lack of a suitable lower temperature channel prevented the imaging DEMs to be extended to lower temperatures. The obtained DEMs have a peak around $\log T(\mathrm{~K}) \sim 6.3$, rapidly decreasing at higher temperatures similar to the spectroscopic studies discussed above. At temperatures lower than $\log T(\mathrm{~K}) \sim 6.3$ the DEM falls with one order of magnitude only that is less than the DEM obtained by Doschek et al. (2010) but similar to Brosius et al. (2008). If the background region emission had been taken into account (see their Fig. 2a and the explanatory text), the fall between $\log T(\mathrm{~K}) \sim 5.8$ and 6.2 would possibly have increased.

\subsection{Relative element abundances}

Skylab observations have first enabled the measurement of the elemental composition by using a simple line ratio of the Mg VI and Ne VI near $400 \AA$ lines that have the same dependence on temperature and density. Sheeley (1995) used some of those data taken in AR and surrounding areas and found that newly emerging plasma along the neutral line in ARs is Ne-rich, while longer lived areas are Mg-rich. The very first indication that the quiet Sun above opposite polarity fluxes, some of which are associated with CBPs, have photospheric abundances comes from the Skylab data analysed by Sheeley (1996). The lack of suitable lines in later missions led to a different approach in establishing the elemental abundances in present day data. As mentioned above the only study to investigate the elemental abundances of CBPs is by Brosius et al. (2008). To obtain the DEM of a CBP (see the previous Sect. 5.7) two sets of elemental abundances were used, the photospheric abundances of Grevesse and Sauval (1998) and the coronal abundances of Feldman et al. (1992). The difference of the two type of abundances is confined in the first ionization potential (FIP) of the different elements with the "FIP effect" that describes the enhancement of low-FIP ions $(<10 \mathrm{eV})$ by a factor of up to $\sim 4$ relative to their photospheric abundances, while coronal abundances of high-FIP ions $(>10 \mathrm{eV})$ are the same as in the photosphere. Only transition region lines that are emitted from low-FIP ( $\mathrm{Mg}$ and $\mathrm{Ca}$ ) and high-FIP $(\mathrm{Ne})$ ions were available for the calculation of the FIP effect in a CBP. A "smoothness and continuity" of the DEM derived from the low-FIP Mg V, Mg VI Mg VII, Ca VII, and the high-FIP Ne IV and $\mathrm{NeV}$ was used as a criterion to select the most suitable abundances. A consistent DEM was derived only when photospheric elemental abundances were used. The study suggests that the photospheric abundances could be produced by reconnection-driven chromospheric evaporation that is also consistent with some of the obtained Doppler shift results in transition region and coronal lines (see Sect. 5.3 for more details). The finding is also consistent with the report that jets from CBPs in polar coronal holes are found to have photospheric abundances (Lee et al. 2015). 


\section{Oscillations in CBPs}

Emission variability is a typical feature of CBPs that are observed in spectral lines with different formation temperatures from the chromosphere to the corona. Coronal oscillations were widely studied during the past few decades and their signatures of propagating waves were intensively investigated both observationally and theoretically. Sheeley and Golub (1979) reported from Skylab/ATM spectroheliogram observations taken in Fe XV 284.16 ̊ over 1.5 day in January 1974 that loops composing the CBPs evolve on a time scale of $\sim 6 \mathrm{~min}$. The intensity variations were found to be periodic. Habbal and Withbroe (1981) and Habbal et al. (1990) also using Skylab/ATM spectroheliograms reported that CBPs exhibit large variations in the intensity of chromospheric, transition-region and coronal lines over time scales as short as the time resolution of their data, i.e., $5.5 \mathrm{~min}$. The variations appeared to be related to substructures in the CBPs, i.e., small-scale individual loops, and no correlation in the intensity variations in spectral lines with different formation temperatures was found. Strong et al. (1992) reported intensity variations in CBPs observed with Yohkoh/SXT that lasted from a few minutes to hours. The results were later confirmed from Hinode/XRT data (Kariyappa and Varghese 2008). Madjarska et al. (2003) used SOHO/SUMER observations in the S VI $933.4 \AA$ line to confirm the intensity variations in CBPs. The data used in the study were taken in a sit-an-stare mode with no compensation for the differential rotation which did not permit any further analysis.

The first investigation on oscillations in CBPs by Ugarte-Urra et al. (2004a) found a period of $491 \mathrm{~s}$ detected in the SUMER/SoHO S VI $933.40 \AA$ line. Intensity variations were found on a time scale of 420-650 s that correlated in the transition-region CDS/SoHO O VI $629.73 \AA$ and O III $599.60 \AA$ lines, and also the He I $583.34 \AA$ line, while the changes in the coronal line Mg IX $368.07 \AA$ occurred on a longer time scale. From the wavelet analysis it was established that the intensity variations "appear in random fashion and sometimes after periods of quietness". In two cases oscillatory behaviour was identified. The authors also examined damped oscillations peaking at $546 \mathrm{~s}$ in one of the CBPs observed in the OVI $629.73 \AA$ line but no corresponding behaviour was detected in the SOHO/CDS He I $584 \AA$ line. Ugarte-Urra et al. (2004b) further investigated CBP oscillations, this time in a SOHO/CDS wide-slit time series in the He I $584.34 \AA$, O V $629.73 \AA$ and Mg VII/IX $368 \AA$, confirming the presence of intensity oscillations in CBPs. Periods with a range between 600 and 1100 s were found that were explained in terms of global acoustic modes of the magnetic loops composing the CBPs (Fig. 32). Periods as short as $236 \mathrm{~s}$ were also identified. These periods belong to the chromospheric oscillation range and were related to the footpoints of the CBP loops.

Using TRACE data taken in the $195 \AA$ and $1216 \AA$ passbands, Tian et al. (2008b) applied Fourier and wavelet analysis searching for long-period oscillations in CBPs. The oscillatory power was found to be stronger in CBPs with respect to the surrounding quiet Sun with different periods in different CBPs. The periods also differed in various parts of the same CBP. From the wavelet analysis oscillations with periods ranging from 8 to 64 min were found that last for several cycles in both the coronal part of the CBPs as well as in their chromospheric footpoints. The results, however, do not provide certainty on whether propagating magneto-acoustic waves or recurrent 

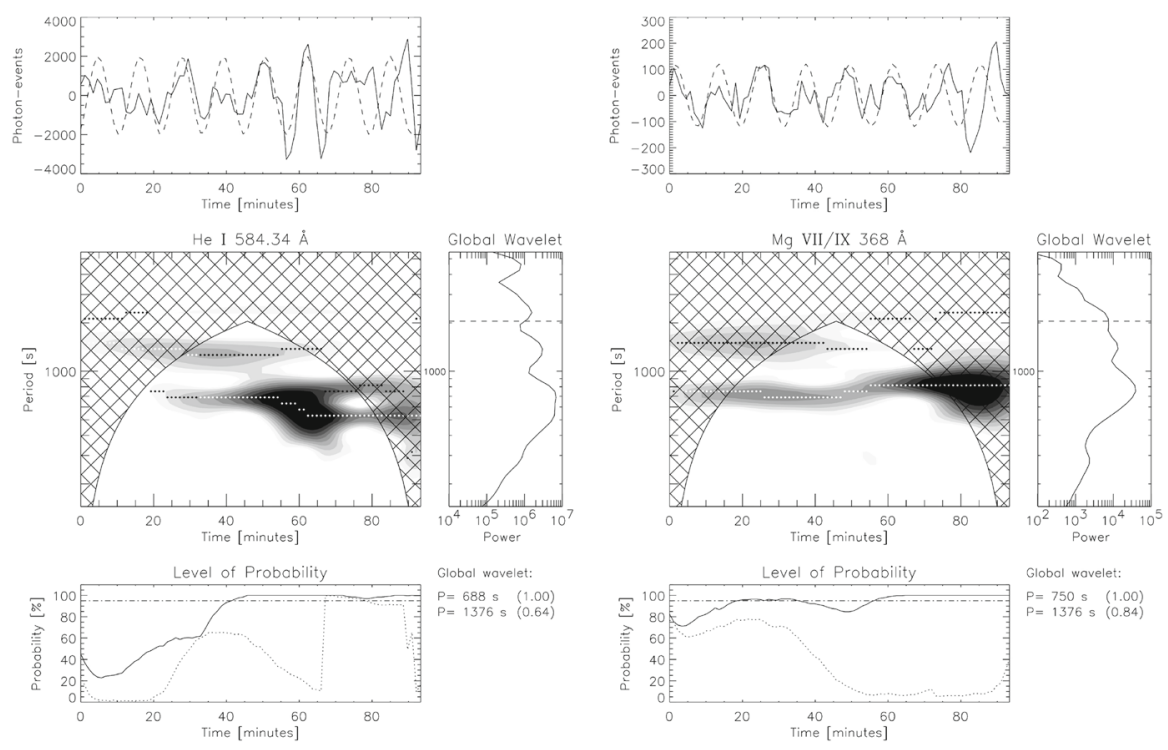

Globol wavelet:

$\begin{array}{ll}P=750 \mathrm{~s} & (1.00) \\ P=1376 \mathrm{~s} & (0.84)\end{array}$

Fig. 32 Wavelet analysis of a CBP. Image reproduced with permission from Ugarte-Urra et al. (2004b), copyright by ESO

magnetic reconnection cause the observed intensity oscillatory behaviour. The doubts come from the fact that the chromospheric oscillation periods are all longer than the chromospheric acoustic cutoff period of $3.7 \mathrm{~min}$ and despite the feasibility that the acoustic cut-off could raise up if it is assumed that the waves propagate along inclined magnetic field lines.

Kumar et al. (2011) analysed Hinode/XRT observations at $30 \mathrm{~s}$ cadence. They also reported oscillations in CBPs with wavelet derived periods ranging from 12 to $60 \mathrm{~min}$. Another interpretation of the intensity variations in CBPs detected in IRIS spectral and imaging observations in the Si IV $1394 \AA$ line is put forward by Samanta et al. (2015). The authors hypothesised that the Si IV line width and intensity enhancements and nonGaussian spectral line profiles are the signatures of transition region explosive events. The explosive-event term is used to describe non-Gaussian profiles in transition region lines and is believed to be a signature of bi-directional flows or a plasmoid propagation resulting from magnetic reconnection. Repetitive reconnection is suggested to explain the quasi periodic nature of the observed brightenings in the CBPs.

To explain oscillations in CBPs, Chandrashekhar and Sarkar (2015) used for the first time a modelling approach by employing a nanoflare heating loop model. The coronal magnetic field of CBP loops were obtained to derive the CBP loop lengths based on SDO/HMI data and by employing a potential field extrapolation model. These were then used in the nanoflare modelling of multi-strand loops. Intensity observables from the forward modelling were obtained and wavelet analysis was applied on the intensity time series. The study found that the derived oscillations also reported in past observations "are actually shock wave propagations along the loop." Hydrodynamic shock waves generated by reconnection events (e.g., nanoflares) can create intensity disturbances that in a particular time range could be identified as oscillations. Different 
intensity background subtractions led to the derivation of adiabatic standing modes both from the observed and simulated data. Therefore, recurrent energy releases in CBPs are possibly the cause for the large range of the reported oscillation periods in CBPs, also suggested by the study of Samanta et al. (2015) discussed above.

\section{Transient phenomena associated with CBPs}

\subsection{Microflaring}

The very first observations of CBPs with the S-054 X-ray telescope on board Skylab revealed that a small area of the CBPs increases its brightness by several orders of magnitude on a time scale of minutes (Golub et al. 1974). The intensity variability had large similarities to solar flares in active regions but on smaller scales, thus the term microflaring was introduced. Golub et al. (1974) established that 5-10\% of the XBPs show microflaring activity that is found in XBPs at all latitudes.

The large variabilities of the X-ray emission from CBPs was later confirmed from Yohkoh/SXT observations (Strong et al. 1992). The observations also showed that microflaring can trigger brightenings in loops larger than the CBPs themselves that "brighten along their lengths" with speeds from 300 to $1000 \mathrm{~km} \mathrm{~s}^{-1}$, actually referring to the later named X-ray jets from CBPs. The study put forward the idea that the microflaring may be caused by magnetic reconnection of emerging magnetic flux with the ambient magnetic field. Eruptions from CBPs are now found to mostly occur following magnetic flux convergence rather than flux emergence (Mou et al. 2018, and the references therein). CBPs produce mini-eruptions (see Sect. 7.2) that in the quiet Sun happen on average $17 \mathrm{~h}$ after the CBP first appearance and take place during the magnetic-flux convergence phase (Mou et al. 2018, and the references therein). This has already been indicated in a study by Harvey et al. (1993), who found that CBPs flare-up after several hours of growth, and some produce several 'flares' during their lifetime, but only $14 \%$ of the sample (514 CBPs) was found to show this explosive behaviour. Shimojo and Shibata (1999) studied the occurrence rate of transient brightenings above the $3 \sigma$ background emission in a CBP ( $30^{\prime \prime}$ size) found near a preceding spot of NOAA 7270. The brightenings were classified as microflares and 92 were identified during the lifetime of the CBP that lasted from 1992 September 1 to 4 . The large number of microflares may be related to the identification threshold criteria. In earlier studies only an intensity increase of a few orders of magnitudes was considered as microflaring. Some of the microflares were associated with X-ray jets or jet-like phenomena. The microflares were found to have a power law distribution with an index of $1.7 \pm 0.4$. This index value taken from a single active-region CBP is similar to the frequency distribution of microflares obtained for active regions (Christe et al. 2008), indicating large similarities with AR flares. Madjarska (2011) reported on a repetitive microflaring related to a X-ray jet from a CH CBP during $17 \mathrm{~min}$ (Fig. 33). Each microflare (energy deposition) was "followed by the expulsion of pre-existing or newly reconnected loops and/or collimated flow along open magnetic field lines" (see the next section on jets from CBPs). 

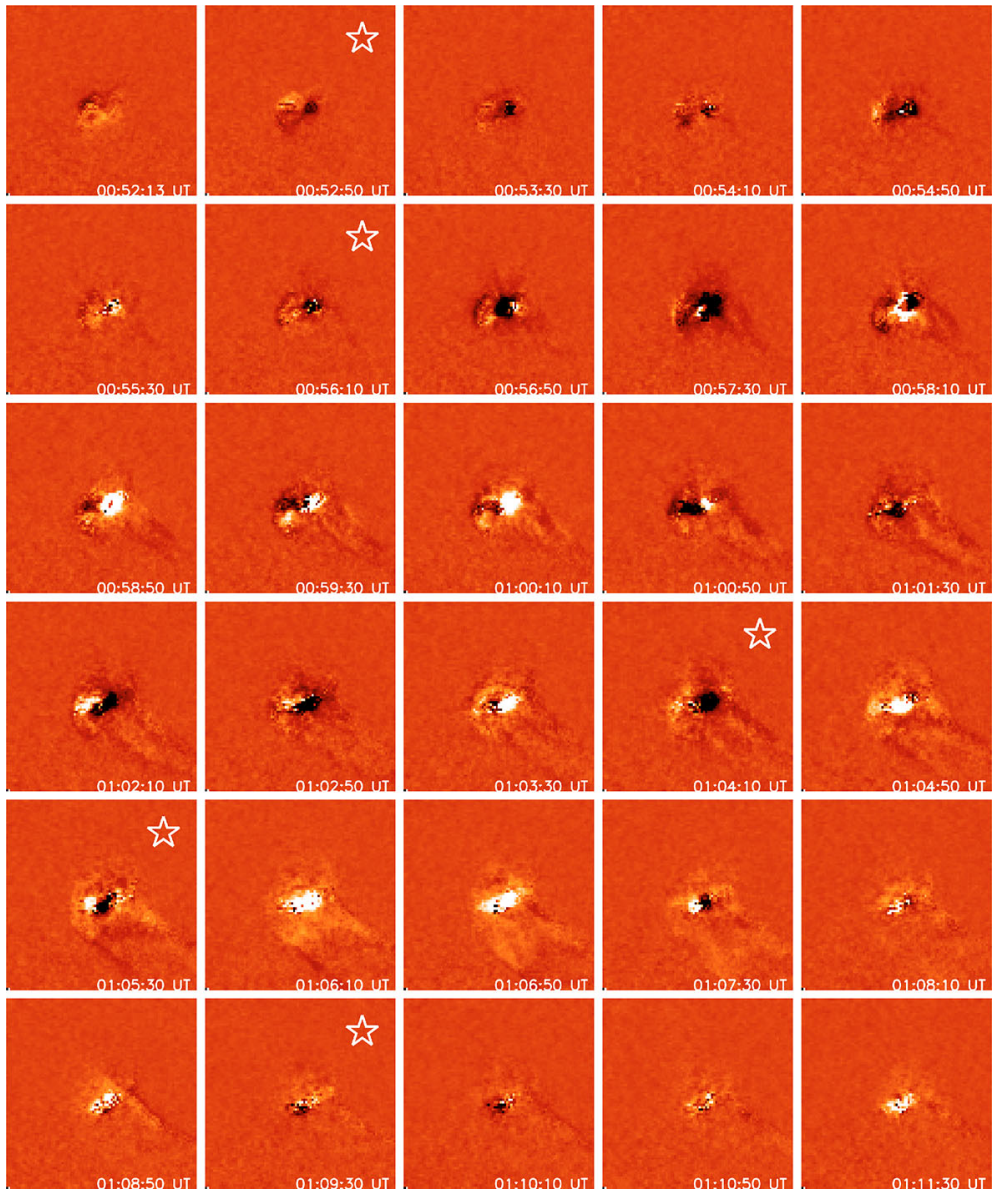

Fig. 33 Series of X-ray (taken with Hinode/XRT) difference images produced by subtracting from each image the precedent one. Stars denote the moments of microflaring, i.e., sudden brightenings seen in the difference images as strong darkenings. Image reproduced with permission from Madjarska (2011) copyright by ESO

The plasma properties of microflares were also studied by Kamio et al. (2011) in the two topologically different solar regions, coronal holes and quiet Sun. Hinode/EIS and XRT data were used where seven QS and three coronal hole CBPs that show microflaring were analysed. The microflares were investigated in Hinode/EIS spectral lines with formation temperatures in the range from $\log T(\mathrm{~K}) \sim 4.7$ (He II) to 6.3 (Fe XV). Simultaneously recorded X-ray images taken with the Al_poly and Be_thin filters at 1 min cadence were also analysed. While the Al_poly filter is sensitive to lower temperatures, the $\mathrm{Be}$ _thin filter registers higher temperature plasma emission and it is usually detected in active regions. The lightcurves of the CBPs were found to produce impulsive peaks in the $\mathrm{X}$-ray emission and spectral lines with high formation temperatures, i.e., Fe XII and FeXV. The first emission peak was followed 8-22 min later by a second peak but this time in the cooler emission (He II and O V, Fig. 27). This 
emission pattern is also commonly observed in large flare loops. The electron density showed an increase at the impulsive peak supporting chromospheric evaporation models. These properties were found both in $\mathrm{CH}$ and QS CBPs. While QS microflares were linked to the formation of dimming (area with depleted emission), diffused jets in $\mathrm{CHs}$ were produced in relation to the microflares. Based on these results, the authors concluded that microflares have common characteristics with AR flares. Zhang et al. (2014) also reported microflaring (named flashes by the authors) in a CBP observed by Hinode/XRT. The authors interpreted the microflarings as "reciprocatory reconnection in the CBP, which means that reconnected loops in the outflow region of the first reconnection process serve as the inflow of the second reconnection process." Ning and Guo (2014) and Li et al. (2016) describe microflarings in what appear to be AR CBPs and associated high velocity bi-directional flows with velocities of 380 and $300 \mathrm{~km} \mathrm{~s}^{-1}$, respectively, trapped in the CBP loops.

\subsection{Eruptions from CBPs: macrospicules, mini-filaments, jets and mini-CMEs}

From co-temporal He II $304 \AA$ spectroheliograms obtained with the Naval Research Laboratory (NRL) experiment on board Skylab and $\mathrm{H} \alpha$ full disk images from BBSO, the link between observations of 'flares' in XBPs and $\mathrm{H} \alpha$ macrospicules was probed (Moore et al. 1977). The analysis showed that most microflares in XBPs often produce $\mathrm{H} \alpha$ macrospicules that are commonly observed in EUV (He II $304 \AA$, Bohlin et al. 1975). These macrospicules appear to be the ejection from CBPs presently known as blowout jets (Raouafi et al. 2016, and the references therein).

Brueckner and Bartoe (1983) were first to identify the presence of EUV jets from Doppler shifts as high as $400 \mathrm{~km} \mathrm{~s}^{-1}$ of transition region spectral lines registered with the NRL's High Resolution Telescope and Spectrograph (HRTS). From X-ray images taken by Yohkoh/SXT, CBPs were found to produce microflares accompanied by plasma ejections with speeds of up to $1100 \mathrm{~km} \mathrm{~s}^{-1}$ along large loops (Strong et al. 1992). Shibata et al. (1992) first reported the observation of X-ray jets in Yohkoh/SXT data that appear as collimated flows, many originating in XBPs or active regions. Later, Hinode/XRT and EIS provided a unique opportunity of observing CBPs and transient phenomena like X-ray jets associated with them (e.g., Culhane et al. 2007b; Cirtain et al. 2007; Subramanian et al. 2010; Madjarska 2011; Madjarska et al. 2012). A dedicated review by Raouafi et al. (2016) describes in detail the properties and modelling of X-ray/EUV jets. However, a unique one-to-one relationship of X-ray/EUV jets and CBPs in all topologically different solar regions, CHs, QS and ARs is still to be confirmed.

Mandrini et al. (2005) reported the smallest magnetic cloud (MC) source-region associated with the ejection of a interplanetary flux rope from a QS CBP on 1998 May 11 located at the disk centre. The CBP was observed to form a sigmoidal structure as seen in projection on the disk following a rotation of one of its polarities. The CBP produced at least three ejections that are also linked to coronal dimmings and a structure that indicated a cusp formation. The ejection is indirectly linked to a small MC registered by WIND 4.5 days later. The association of the $\mathrm{MC}$ with the $\mathrm{CBP}$ eruption was based on a few observed properties. The CBP was located at the disk centre 
and any related eruption that propagates in radial direction would reach the WIND spacecraft. The timing and the orientation of the MC axis is similar to the direction of the CBP sigmoidal structure. Furthermore, the sign of the magnetic helicity of both events appear to be in agreement. A statistical study based on STEREO/SECCHI COR1 white light images, (Paraschiv et al. 2010) demonstrated that CBPs are the source regions of white light jet-like ejections over a large range of latitudes including quiet Sun regions. More than 10,000 white-light jets were identified and some were clearly traced back to CBPs. The projected speeds of 106 jets were found to have velocities in the range from 180 to $380 \mathrm{~km} \mathrm{~s}^{-1}$.

From their study on microflares in CBPs, Kamio et al. (2011) established that microflares are associated with jets in $\mathrm{CHs}$ and dimmings in the QS. The difference comes from the different configuration of the background magnetic field. The CHs are dominated by open field which results in collimated flows (jets) from CBPs, while the dimmings in the QS are caused by the removal of the overlying coronal magnetic field leading to the formation of density depleted region.

Using SDO/AIA observations that monitor continuously the solar atmosphere at 12 s cadence, Hong et al. (2014) studied the dynamic behaviour of 30 CBPs in a coronal hole. One-quarter to one-third of all CBPs were found to have one or more mini-filament (MF) eruptions and EUV jets. The MF eruptions were associated with a localised brightness increase, i.e., a microflaring, slow rising motion followed by eruption of hot and cool material (e.g., Fig. 34). The authors concluded that convergence and cancellation of magnetic bipoles associated with the CBPs are possibly responsible for the erupting MFs confirming the conclusions reached by Hermans and Martin (1986).

Innes et al. (2009, 2010) first introduced the term mini-CMEs as small-scale eruptions from the quiet Sun found at the junctions of supergranulation cells. The majority of the mini-CMEs showed cool plasma ejections. Podladchikova et al. (2010) also studied mini-CME events in the SECCHI/EUVI $171 \AA$ channel. The study concluded that the mini-CMEs are characterised by smaller sizes and shorter lifetimes with respect to the classic CMEs. Small-scale coronal waves and dimmings were both associated with mini-CMEs. These studies, however, did not investigate mini-CME events in the context of CBPs although the presented observational material indicates that possibly many or even all mini-CMEs occur in CBPs.

Ulyanov et al. (2010) also reported the ejection of coronal material with velocities in the range $80-110 \mathrm{~km} \mathrm{~s}^{-1}$ from CBPs identified in $5 \mathrm{~s}$ cadence observations in the $171 \AA$ channel of CORONAS-FOTON instrument TESIS. Recently, Mou et al. (2018) showed that $76 \%$ of quiet Sun CBPs (31 out of 42) produce at least one eruption during their lifetime. The eruptions occurred late in the CBPs lifetime at $\sim 17 \mathrm{~h}$ after a CBP formation for an average lifetime of CBPs in SDO/AIA $193 \AA$ of $\sim 21 \mathrm{~h}$. Generally, convergence and cancellation of magnetic flux take place at the time of the eruptions. Chromospheric material, in some cases clearly distinguishable as a mini-filament, is usually ejected together with the CBP or higher overlying hot loops. Microflaring is always associated with this dynamic process. In most eruptions, the cool erupting plasma obscures partially or fully the micro-flare until the erupting material moves away from the CBP. From 21 eruptions 11 are found to produce mini-CMEs. A mini$\mathrm{CME}$ is identified when two of the three components of a classic CME are present: 

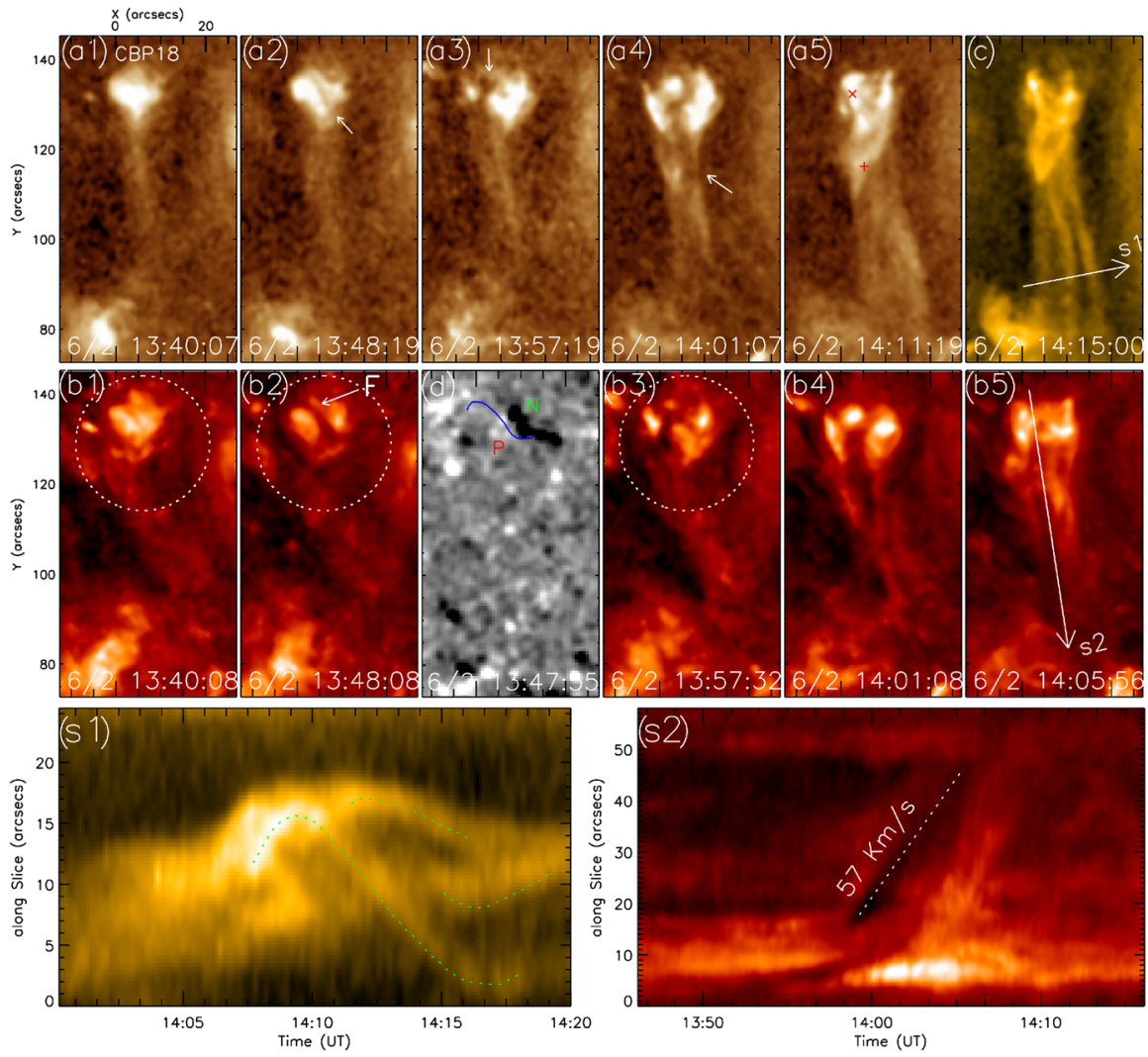

Fig. 34 Top row: a1-a5 SDO/AIA $193 \AA$ images of a jet from a CBP with an arrow indicating an eruptive mini-filament, c SDO/AIA $171 \AA$ image of the jet. An arrow s1 marks a image-cut from which a time-slice image is produced and shown in the left panel of the bottom row. Middle row: b1-b5 SDO/AIA $304 \AA$ Amages of the jet and mini-filament; d SDO/HMI longitudinal magnetogram. A dot-line circle indicated the CBP. Bottom row: SDO/AIA $171 \AA$ A time-slice image (left panel) along the arrow s1 in panel c. SDO/AIA $304 \AA$ time-slice image (right panel) along the arrow s2. Image reproduced with permission from Hong et al. (2014), copyright by AAS

bright expanding loops ahead of a dark region, and eruptive mini-filament or cool plasma cloud. In addition coronal waves were detected in three cases. Figure 35 shows an example of one of the identified mini-CMEs with a microflaring core and a dimming region. Innes et al. (2009) concluded that 1400 mini-CMEs may occur per day over the whole Sun, while Mou et al. (2018) estimated that a minimum of 870 eruptions from CBPs should happen if "CBPs produce at least one eruption during their lifetime".

To conclude, CBPs are the source region for the occurrence of eruptions seen in chromospheric spectral lines or imagers with chromospheric and/or transition region temperature response, named macro-spicules presently known as blowout jets. In coronal holes CBPs produce collimated flows seen in EUV and X-rays, i.e., jets. In the quiet Sun almost $80 \%$ of CBPs produce eruptions that trigger the appearance of dimming seen in the coronal channels of EUV imagers and expanding loops that brought the introduction of the notion mini-CME. Mini-filaments are usually observed to erupt 

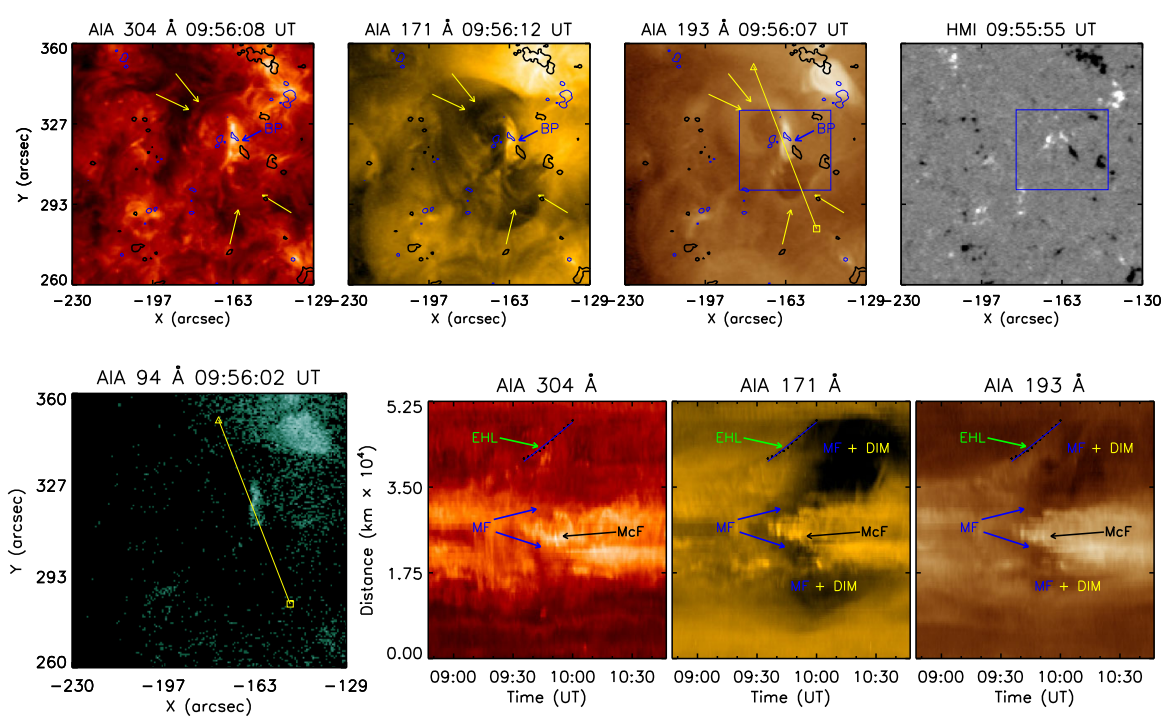

Fig. 35 Top panels: from left to right: AIA $304 \AA, 171 \AA$ and $193 \AA$ images of an eruption from a CBP identified as mini-CME, and a corresponding HMI longitudinal magnetogram scaled from -50 to $50 \mathrm{G}$. The yellow arrows point at the erupting mini-filament.The blue and black contours outline the positive and negative fluxes at $50 \mathrm{G}$. Bottom: AIA $94 \AA$ image showing the microflare. The yellow solid line denotes the slice which has been extracted to produce the time-slice panels in the AIA $304 \AA, 171 \AA$ and $193 \AA$ channels. The bottom of the slice is marked with a triangle and the top with a square. $B P$ bright point, $E H L$ eruptive hot loops, $M F$ mini-filament, $M c F$ micro-flare, and DIM dimming. Image reproduced with permission from Mou et al. (2018), copyright by ESO

during the mini-CMEs occupying parts of the dimming regions or being part of the jet outflows in coronal holes.

\section{Energetics}

Estimation of the CBPs energy losses from radiation and thermal conduction provides valuable information that can help identify the energy sources that are responsible for the CBP existence and evolution. Habbal and Withbroe (1981) addressed the energetics of CBPs in their study on the spatial and temporal variation of the emission from CBPs in EUV spectral lines. They obtained the emission measure, electron pressure and cooling times using coronal $(\mathrm{Mg} \mathrm{X})$, transition region ( $\mathrm{C}$ II and $\mathrm{O}$ VI) and chromospheric $\left(\mathrm{L}_{\alpha}\right)$ lines. The emission variations in the $\mathrm{L}_{\alpha}$ line was considered to have been produced from the heating of the coronal plasma with the energy that is carried into the chromosphere from the corona by thermal conduction triggering emission in the chromospheric $\mathrm{L}_{\alpha}$ line. Under this assumption the total rate of energy loss for one CBP in their study is estimated at $1.4 \times 10^{24} \mathrm{ergs} \mathrm{s}^{-1}$ that gives cooling times between 100 and $350 \mathrm{~s}$ that are in agreement with the temporal rate of the intensity variation of the EUV lines from CBPs in their study, i.e., in average $5.5 \mathrm{~min}$. The study concludes that "the intensity variations are caused by intermittent, impulsive heating of the coronal 
plasma in the miniature loops making up the bright point which cools by radiation and thermal conduction".

From the study of six CBPs observed by SOHO/EIT and MDI, and Yohkoh/SXT, Preś and Phillips (1999) established that conductive energy losses are at least one order of magnitude higher than radiative losses, and the total energy losses are within a factor of 2 larger than the calculated magnetic energy. The total radiative and conductive losses estimated from the SOHO/EIT $195 \AA$ channel over the full lifetime of the CBPs range from $5.6 \times 10^{27}$ to $1.1 \times 10^{28} \mathrm{ergs}$ (only $10 \%$ of the total losses), and from $4.0 \times 10^{28}$ to $2.6 \times 10^{29} \mathrm{ergs}$, respectively. The magnetic energy is estimated to be between $2.0 \times 10^{28}$ and $2.4 \times 10^{29}$ ergs assuming a field configuration in the form of a semicircular loop with an uniform cross section and an area equal to the mean of the two areas of two polarities where the loop is rooted. A Spitzer conduction is assumed with the thermal energy being transported to the chromosphere along loopshaped field lines forming the magnetic skeleton of the CBPs. Given the uncertainties of the calculation of the magnetic energy and energy losses, the authors reached the conclusion that essentially all the energy losses of the CBP plasma is supplied by the magnetic energy.

Ugarte-Urra et al. (2004b) concluded that energy supply from a helicity injection in a CBP, assuming binary reconnection as heating mechanism suggested by Priest et al. (2003), would provide only energy in the range of $\sim 10^{18}-10^{20} \mathrm{ergs} \mathrm{s}^{-1}$ which is much smaller than the energy loss in CBPs of $\sim 10^{23}-10^{24} \mathrm{ergs} \mathrm{s}^{-1}$. Therefore, it is an insufficient energy source. The magnetic flux value of the analysed CBP was in the range from $7.8 \times 10^{17}$ to $7.2 \times 10^{18} \mathrm{Mx}$, while magnetic flux of $1.4 \times 10^{20} \mathrm{Mx}$ would be required for this mechanism to be efficient in heating the CBP.

\section{Modelling}

The emission from CBPs and its variability is believed to be sustained by a continuous steady heating input while sudden intensity variations are thought to be caused by an impulsive energy release. Theoretical modelling has followed in the steps of the observational findings on CBPs. It includes analytical analysis that have evolved into 2D, 2.5D and finally 3D MHD models. Several of these models have some of the initial boundary conditions derived from magnetic field observations. Observables obtained from theoretical modelling have also been compared with imaging observations to narrow the possible range of mechanisms and the background environment in which these phenomena take place. Each of the reported models developed over the past five decades have had an important contribution on the growing knowledge of possible plasma heating mechanisms in small scale coronal loops, i.e., CBPs. The convergence of bipolar magnetic fluxes of opposite polarities has been an essential feature on which several 2D and 3D models have been produced. In all these models magnetic reconnection in the corona is driven by converging photospheric footpoint motions which produces a CBP. Although these models have replicated some of the observational features of CBPs known at the time of their development, they do not reflect new observational facts like, for instance, the appearance of CBPs during magnetic flux emergence and divergence. Another series of studies investigated how various hori- 
zontal footpoint motions including shearing, convergence and fragmentation lead to the formation of electric currents in the chromosphere, transition region and corona. The dissipation of these currents in specific locations defined by the footpoint motions created localised heating at the locations of the observed CBPs. In the following the main setup and achievements of these models are presented.

Nolte et al. (1979) investigated the emission variability of seventeen X-ray BPs in Skylab data taken at $90 \mathrm{~s}$ time cadence. The intensity decay of the CBPs was interpreted as caused by a "sudden turn-off of the steady heating". The study also reports the observation of strong brightenings (microflarings) in CBPs before their disappearance presently known as CBP eruptions (Hong et al. 2014; Mou et al. 2018). The authors speculated that two mechanisms should be considered for the CBP generation and sudden disruption: one that provides a steady heating like for active region loops and one that can explain the occurrence of impulsive events leading to the distraction of the magnetic skeleton that holds the CBP plasma.

Various models have been developed since the first CBP observations in X-rays. Parker (1975) introduced the very first model of CBPs where CBPs result from the rising of an elementary flux tube through the solar atmosphere by magnetic buoyancy. In this model twisting by photospheric motions leads to the accumulation of the azimuthal field at the loop apex, and after a certain critical value is reached any simple equilibrium ceases, the tube kinks and twists. The kinked field is dissipated through magnetic reconnection which leads to the formation of an XBP. Glencross (1975) put forward the idea that CBPs consist of flux ropes that are build up by twisted braids wrapped around one another creating conditions for the formation of current sheets. Braid reconnection would then provide enough heating to maintain the plasma temperature above $10^{6} \mathrm{~K}$. During this process instabilities can lead to microflaring in CBPs. Waldron and Mullan (1987) developed a 2D MHD model to investigate how a chance encounter of opposite directed magnetic-field lines suggested by Harvey (1985) could affect the stratified atmosphere.

\subsection{Ad-hoc heating models}

Kankelborg et al. (1996) analysed data from the Multi-Spectral Solar Telescope Array (MSSTA) sounding rocket payload taken with the large Herschelian telescope in a Fe XII $193 \AA$ passband, a Ritchey-Chrétien telescope with a passband centred at H Ly $\alpha 1216 \AA$ and a Cassegrain telescope with a spectral response centred at $173 \AA$ that is dominated by Fe IX $171 \AA$. Co-temporal Kitt Peak longitudinal magnetograms were also used. The footpoints of CBPs were clearly seen in Ly $\alpha$ as well as their bipolar magnetic field counterparts. A one dimensional loop model (see their Fig. 5) was fitted to the magnetic field data showing that if the footpoints are not too narrow, thermal conduction from the corona can inject enough energy through the $10^{5} \mathrm{~K}$ layer to account for the emission flux in the $\operatorname{Ly} \alpha$ network where the CBPs are rooted. Kankelborg et al. (1997) extended the study to 23 CBPs for which the temperature, pressure and heating rate were deduced. Again assuming that thermal conduction is responsible for the observed Ly $\alpha$ emission at the footpoints of the CBP loops, the loop cross-section and the conductive flux values were obtained from the same 
one dimensional model. Evidence was found for a systematic imbalance between the conductive and radiative losses at the footpoint area which can be due to the above mentioned assumption, or it is a natural consequence of the CBP loop heating.

The time-dependent zero-dimensional, enthalpy-based thermal evolution of loops (EBTEL, Klimchuk et al. 2008) hydrodynamic coronal model was used by Chitta et al. (2013) to investigate nanoflare heating of CBP plasma. Although the paper does not use the term coronal bright point, the analysed quiet Sun small-scale coronal loop events are actually CBPs (see Fig. 1 in their paper). The observation (SDO/AIA) derived DEMs (see Sect. 5.7) were compared with the model derived DEMs. Three different heating models are tested: low frequency, medium frequency and medium frequency hybrid models. The study concludes that a "medium-frequency heating model with a population of different heating amplitudes can roughly reproduce the observations". The study also explores a loop model with a non-uniform cross-section of the loop along its length and steady heating, and finds that this model can also reproduce the observed DEMs. To a large extent the study is inconclusive and suggests that further observational constraints are needed to better understand the nature of coronal heating of small scale loops in the quiet Sun.

\subsection{Convergence and cancellation models}

Following the observational evidence on the relationship of CBPs with converging magnetic fragments of opposite polarities, Priest et al. (1994) proposed a model, called Converging Flux Model (CFM), aimed at explaining these observations. The initial setting of the model includes two magnetic flux concentrations of opposite equal strength polarities that are disconnected by the presence of an overlying horizontal coronal magnetic field. The model acquires three main phases (see Fig. 36). During phase (1), named the pre-interaction phase, the two magnetic fragments converge driven by the horizontal photospheric flow reaching a certain distance, $d$ [called interactive distance, Fig. 36, panel (i)], and the field lines from these magnetic polarities come into contact forming a null point in the photosphere [panel (ii)]. The flux concentrations continue their approach during phase (2), the interaction phase, while the null point lifts up to form an X-type null point in the corona reaching a maximum height that is half the interactive distance $d$. As reconnection in the null point releases energy in the coronal domain, an XBP forms [panel (iii)] and plasma is ejected along the newly reconnected field lines [panel (iv)]. Next, the null point moves down to the photosphere [panel (v)] and the fragments come into contact that causes magnetic reconnection in the photosphere which is the cancellation phase [panel (vi)]. The interaction phase (coronal reconnection phase) and the cancellation phase overlap in time. This occasionally results in the formation of a small filament above the cancelling features that erupts over an hour later.

Parnell et al. (1994a) further explored the formation of CBPs in the context of the CFM using observations from the Normal Incident X-ray Telescope (NIXT) on board a rocket flight in 1991 and co-temporal Kitt Peak longitudinal magnetograms. For the first time the magnetic topology of two observed XBPs was derived using a low number of point sources to reproduce the $3 \mathrm{D}$ structure of the magnetic field. 

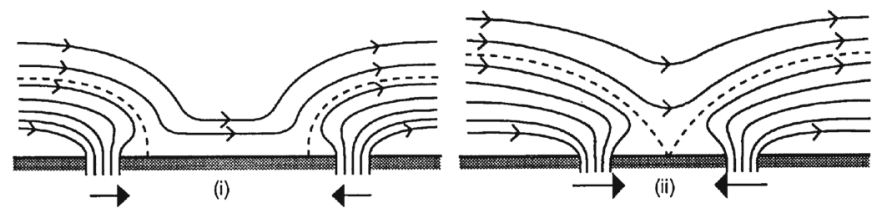

PRE-INTERACTION PHASE

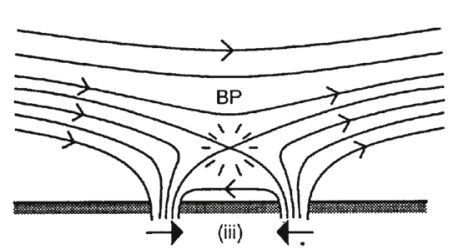

INTERACTION PHASE

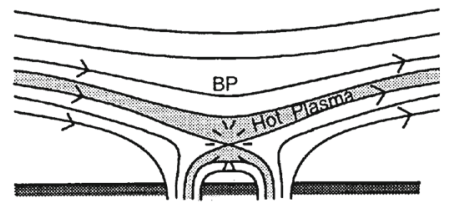

(iv)

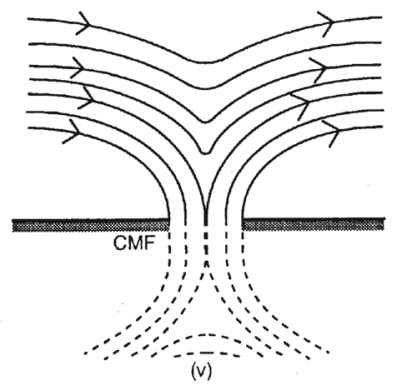

(v)

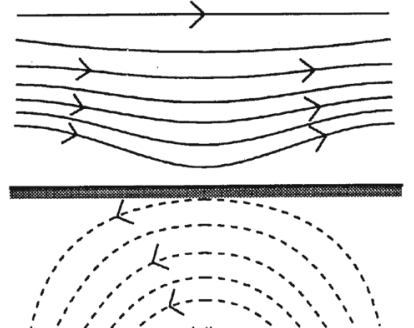

(vi)

CANCELLATION PHASE

Fig. 36 Cartoon describing the converging flux model. Image reproduced with permission from Priest et al. (1994), copyright by AAS

The movement of these point sources leads to brightenings as a result of reconnection between close-by flux regions. The CFM was further explored by Parnell et al. (1994b) using a 3D model of converging fluxes for the interaction of two opposite polarities of different strength. The three phases of CFM are followed with an X-type null point height that varies as the distance of the flux sources changes as well as their strength. A total free energy of the order of $10^{20}-10^{21} \mathrm{~J}$ is produced which is sufficient for a $\mathrm{CBP}$ to reach coronal temperatures. Coronal magnetic reconnection results in Ohmic heating producing a CBP that is also associated with 90\% flux cancellation.

Von Rekowski et al. (2006a) also investigated the converging and submerging flux scenario but with an emphasis on exploring the full time evolution of cancelling magnetic features and their role in a coronal heating event, i.e., a CBP. Observers use the term "cancellation" to describe a continuous common loss of magnetic flux from two opposite signed approaching flux concentrations until the full disappearance of the smallest of these. This information is deduced from magnetogram data obtained from spectral lines that form either at photospheric or chromospheric heights. In modelling, magnetic flux cancellation usually refers to the creation of a new linkage caused by the 
reconnection of approaching opposite fluxes. If this happens above the photosphere, it often leads to the formation of post-reconnection small loops. These loops may subsequently submerge below the photosphere, which, as a secondary effect, will be seen in the magnetograms as flux cancellation. However, reconnection above the photosphere would not necessarily imply co-temporal flux disappearance in magnetogram data, i.e., cancellation. Von Rekowski et al. (2006a) employed a 2D MHD model where the bipolar sources are partially connected so that they marginally touch at the base. The model includes four sources of equal size, field strength and flux distribution, with two inner and two outer sources of opposite polarities. The outer sources connect above to form the overlying ambient magnetic field. The energy calculations suggest that as a result of the reconnection, the magnetic energy is converted to thermal energy through Ohmic heating. A positive Poynting flux across the base is found to contribute only $10 \%$ of the energy input. A magnetic flux decrease, i.e., cancelling magnetic features (CMFs), is therefore interpreted as a signature of elementary heating events. Similar results were obtained in the second part of this study where disconnected converging magnetic bipoles were studied (von Rekowski et al. 2006b).

\subsection{Separator reconnection models}

Observation from Yohkoh/SXT and magnetograms from several groundbased observatories were used by Mandrini et al. (1996) to model a CBP formed following bipolar flux emergence in the vicinity of a decaying active region. The $\mathrm{CBP}$ photospheric magnetic field was extrapolated with a linear force-free model and its temporal evolution was followed during the $16 \mathrm{~h}$ lifetime of the CBP. Quasi-separatrix layers (QSL, Priest and Démoulin 1995) were identified located between the CBP loop system and the pre-existing coronal fields seen as faint X-ray loops. The QSLs' widths were found to be very small during the CBP lifetime $(\sim 100 \mathrm{~m})$ which become larger $\left(\leq 10^{4} \mathrm{~m}\right)$ after the CBP disappearance. Flaring in the $\mathrm{CBP}$ is followed by bright emission that is seen to propagate with a speed of $\sim 670 \mathrm{~km} \mathrm{~s}^{-1}$ from the location of the XBP along the large faint X-ray loop at one side of the QSL with the XBP loops lying on the opposite site suggesting an energy release at the QSL location between two different magnetic flux systems. The study concludes that QSLs are where magnetic reconnection takes place to provide a steady heating that maintains the CBP bright emission in X-rays as well as where sudden larger energy is released causing the flaring and plasma outflows in the vicinity of CBPs.

Longcope (1998) used the minimum current corona (MCC) model of Longcope (1996) to reproduce a CBP associated with a magnetic bipole. The MCC model estimates the current forming on a separator due to displacement of photospheric flux which allows to model coronal evolution through a series of quasi-static states. It is based on observed quantities like CBP loop length and location, temperature, background emission, emission measure etc. The model predicts the interaction distance and the heating power for magnetic bipoles in various configurations as well as the apparent angle between the axes of the bipole and the CBP loops.

A forward modelling was used by Kankelborg and Longcope (1999) to study the reconnection driven heating of a $\mathrm{CBP}$. The $3 \mathrm{D}$ magnetic structure of a $\mathrm{CBP}$ observed 

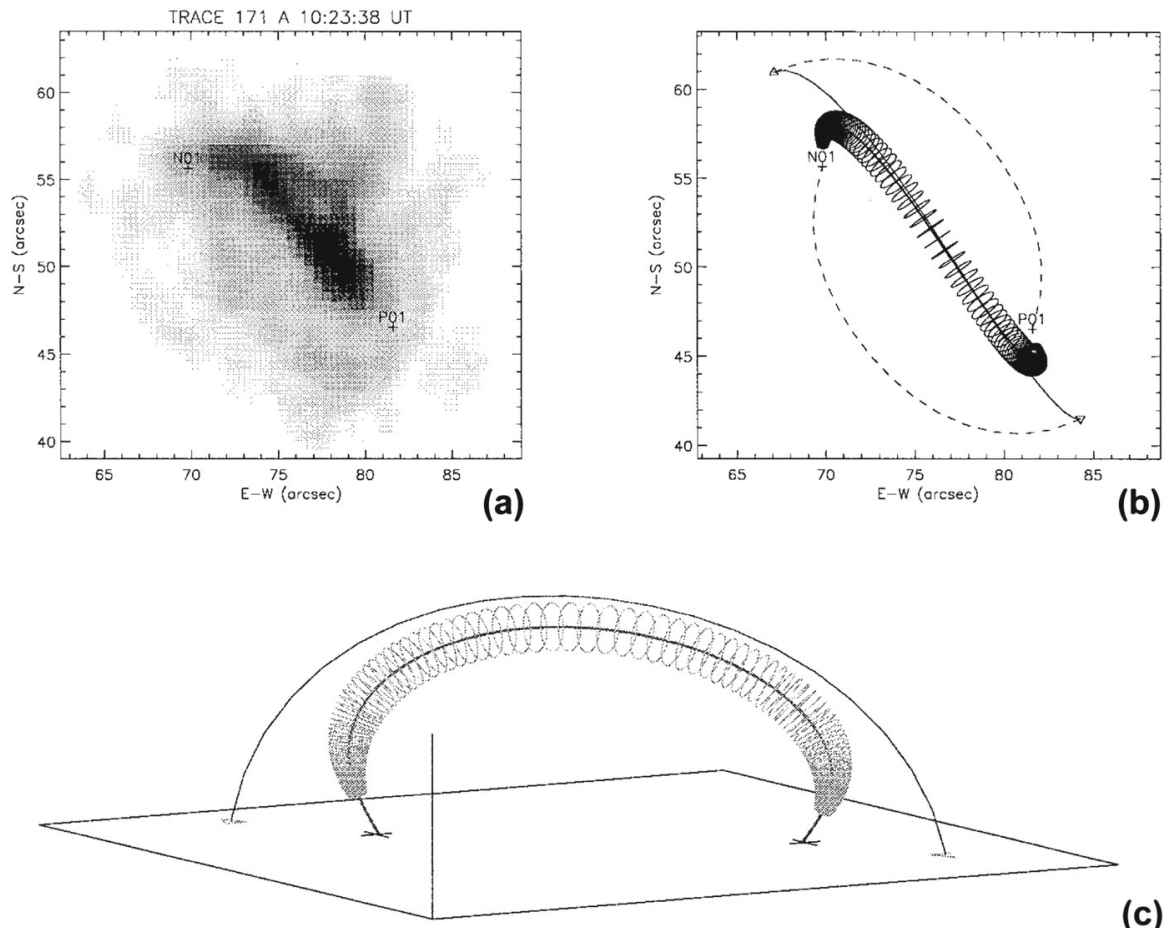

(c)

Fig. 37 The model flux tube. a TRACE $171 \AA$ image of a CBP seen as single loop. b The modelled flux tube viewed with the same projection as in TRACE. Circles show the boundaries of the finite volume elements, which are concentrated toward the end of the tube. c A different view of the model flux tube. Image reproduced with permission from Kankelborg and Longcope (1999), copyright by Springer

in EUV by TRACE was reconstructed using a potential field model on SOHO/MDI magnetograms (see Fig. 37). The MCC model was used to derive the energy associated with reconnection. This energy was applied to drive a gasdynamic model of evolution within a flux tube obtained from the MCC model. The calculated EUV emission, however, was only one fourth of the observed in the TRACE channels, which made the authors conclude that the MCC model fails to explain the observations. Alternatively to the MCC model, a 3D MHD experiment by Galsgaard et al. (2000), Parnell and Galsgaard (2004), and Galsgaard and Parnell (2005) investigated different scenarios of opposite signed flux concentrations that move past one another while their 3D magnetic flux domains are forced to interact. Energy is released through magnetic reconnection in a separator current sheet that forms between the two independent flux systems (Fig. 38). The complicated reconnection process where a recirculation of flux is more than twice the minimum amount of reconnecting flux forms low lying hot loops that could represent a CBP.

Longcope et al. (2001) further explored the hypothesis that magnetic reconnection maintains the heating of CBPs using SOHO/EIT images and co-temporal magnetogram observations from SOHO/MDI employing the three-dimensional reconnection model described in Longcope (1998). For comparison, a simpler model is also intro- 

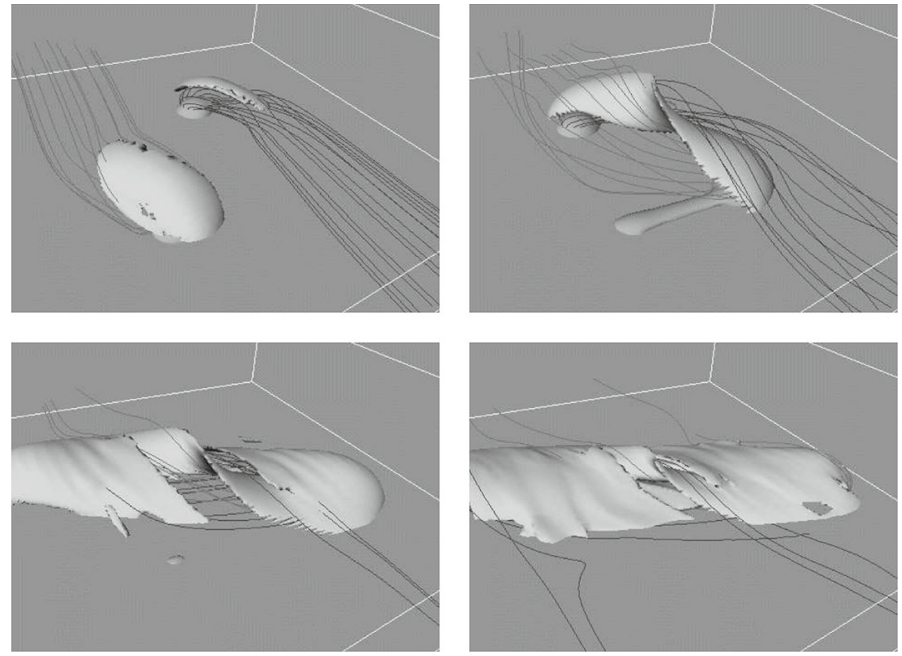

Fig. 38 Panels showing the time evolution $(t=1.46,5.43,9.61,12.58)$ of the current isosurfaces and magnetic field lines. Image reproduced with permission from Galsgaard et al. (2000), copyright by ESO

duced, termed 'a naive bipole model', where a CBP represents a simple loop connecting the two opposite flux polarities that is heated by an unspecified internal mechanism. The naive model predicts a loop whose properties are entirely defined by the bipole alone. Out of 764 CBPs 285 were analysed and some of their observable properties were derived to be compared with the MCC and the naive bipole model. The reconnection model is found to better fit the observations than the naive bipole model giving further support for a reconnection driven heating of CBPs. The orientation of the observed CBPs were found to fit the orientation of the separator rather than the magnetic loops. A general description of the MCC model is shown in Fig. 39. The bipole geometry and background field are illustrated in Fig. 39a, while the emission associated with the CBP along the separator is shown in Fig. 39b. The reconnection process is illustrated in Fig. 39c, d. The background magnetic field is assumed horizontal.

Brooks and Warren (2008) used Kitt Peak longitudinal magnetogram data to compute the coronal magnetic field employing a potential field model. The extrapolated field lines were populated with solutions to hydrostatic loop equations based on a different parameterization of the heating function that scales as $\overline{\mathrm{B}} / \mathrm{L}$, where $\overline{\mathrm{B}}$ is the magnetic field strength averaged along a field line and $\mathrm{L}$ is the loop length. The computed densities and temperatures were used to synthesize EUV and soft X-ray intensities and compare with Yohkoh, SOHO/CDS and SOHO/EIT observations. The best-case model that includes apex heating of expanding loops could reproduce the high-temperature emission and the general morphology of the low-temperature emission, with the average intensities of the analysed spectral lines within 20\%. The morphology of the synthesized EUV images differ from the observations, including the location of the peak emission. A suggested explanation is that the disagreement may be caused by either non-potentiality or dynamically evolving magnetic fields, although the departure from potential field should not be large as shown in Sect. 4.2. 


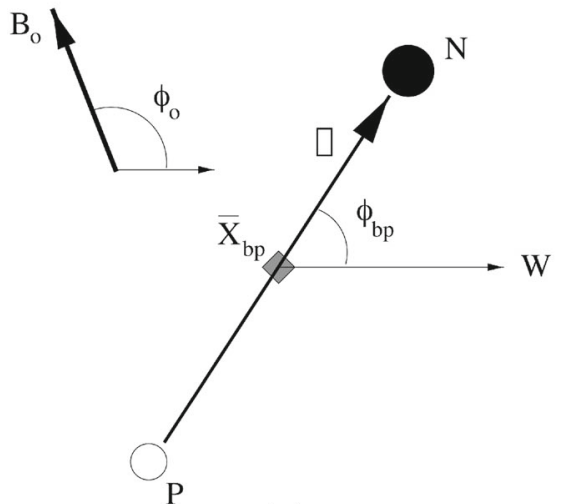

(a)

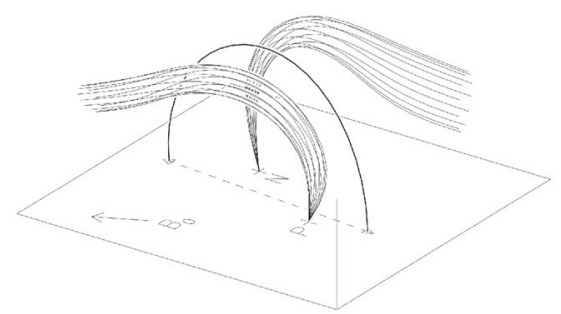

(c)

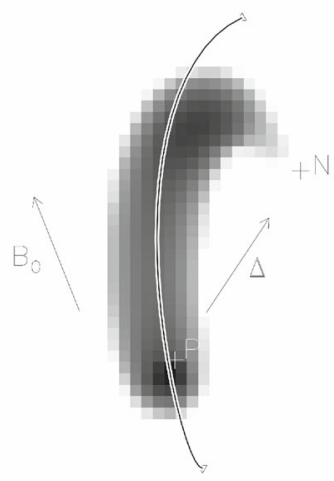

(b)

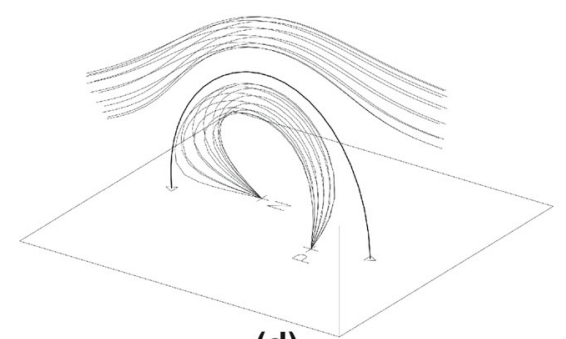

(d)

Fig. 39 MCC model of an XBP. a The geometry of the magnetic bipole and the background field $\mathbf{B}_{0}$. b Emission from flux tube after reconnection. The solid curve shows the separator and the arrows indicate the direction of the bipole orientation $\Delta$. $\mathbf{c}$ Field lines connecting each of the poles to the background field $\mathbf{B}_{0}$ (its direction shown with an arrow in the photospheric plane). The separator is shown as a thick curve. d Post-reconnection field lines. Image reproduced with permission from Longcope et al. (2001), copyright by AAS

Recently, Wyper et al. (2018) developed a model to explain the formation of CBPs in coronal holes and jets. The initial setup of the model is a region seeded with a minority magnetic flux concentration embedded in a majority opposite polarity concentrations, a photospheric magnetic flux pattern commonly found in coronal holes. This photospheric magnetic flux configuration typically produces a fan-spine topology with a single $3 \mathrm{D}$ null-point where the null-point spine axis connects to the minority polarity flux as found in Galsgaard et al. (2017). The minority polarity concentration was stressed by applying three different type of flows- - "a large-scale surface flow that shears part of the separatrix surface only, a large-scale surface flow that also shears part of the polarity inversion line surrounding the minority flux, and the latter flow setup plus a 'flyby' of a majority-polarity concentration past the moving minoritypolarity element". Various CBP morphologies resulted from these. The shearing of the magnetic field near the separatrix produced steady interchange reconnection modulated by quasi-periodic, low-intensity bursts of reconnection. This would manifest as a CBP with periodically varying intensity (see Sect. 6 concerning CBP intensity oscillations possibly caused by intermittent reconnection). Strong shearing near the 
polarity inversion line resulted in a filament channel formation and mini-filament eruptions producing jets through a breakout mechanism. The 'flyby' driving resulted in more energetic jets.

\subsection{Current-sheet dissipation induced by footpoint motion models}

Santos et al. (2008) used 3D MHD modelling to investigate how different patterns of photospheric plasma motions could generate electric currents (Büchner 2006) in the solar atmosphere and how these currents relate to the existence of CBPs. The work is a continuation of an initial study by Santos and Büchner (2007) of a CBP observed by SOHO/EIT and MDI. The studies use MDI magnetograms to obtain the initial boundary condition by extrapolating the longitudinal magnetic field with a potential field model. Local-correlation tracking was applied to derive the pattern of the magnetic flux motions. In Santos et al. (2008) two more velocity patterns were employed derived from the temporal evolution of the CBP for a time period that follows the one analysed in Santos and Büchner (2007). In the simulation some of the horizontal motions are discarded as they are interpreted as emergence, while others are approximated by using a combination of vortices of velocity. The study finds three different patterns, namely shearing, convergence and fragmentation, that give rise to electric currents mainly in the chromosphere but also in the transition region and the corona (below 7.5 Mm). The plasma motions do no create currents everywhere but only at QSLs, where the magnetic field connectivity changes dramatically. In the experiment the electric currents develop generally in the same region regardless on the applied plasma motion, and the area of the CBP coincides with the enhanced currents (Fig. 40).

Heating from adiabatic compression and electric current dissipation were investigated for their role in the formation of CBPs by Javadi et al. (2011). The study is a continuation of the work by Santos et al. (2008) investigating the energy conversion and budget in magnetic flux tubes. The simulations used the LINMOD3D resistive code with a stratified model atmosphere covering the region from the chromosphere into the corona. The energy source for the model is derived from the photospheric plasma motions. Adiabatic compression caused by the magnetic pressure force induced by the plasma motions is found to have a dominant effect in heating the CBP plasma with only a minor role played by Joule current dissipation. This is even more prominent after the temperature of the CBP starts to increase. The role of Joule heating brought up by current dissipation due to anomalous resistivity in the heating of CBP was explored by Adamson et al. (2013) using the resistive MHD simulation code MPSCORONA3D that allows investigating resistive effects within the corona over a wide range of Reynolds numbers. The code uses four main types of resistivity models, namely constant and homogenous resistivity, with Spitzer value of resistivity as well as various models of current-carrier velocity dependent resistivity. The study establishes that the thermal energy increase in CBPs can be caused by DC current dissipation only under the assumption of an unrealistically low critical current-carrier velocity at which turbulent resistivity is switched on. Generally, the corona is energized by plasma compression when more realistic resistivity models are used, concluding that Joule heating in the solar corona strongly depends on the magnitude of the resistivity. 

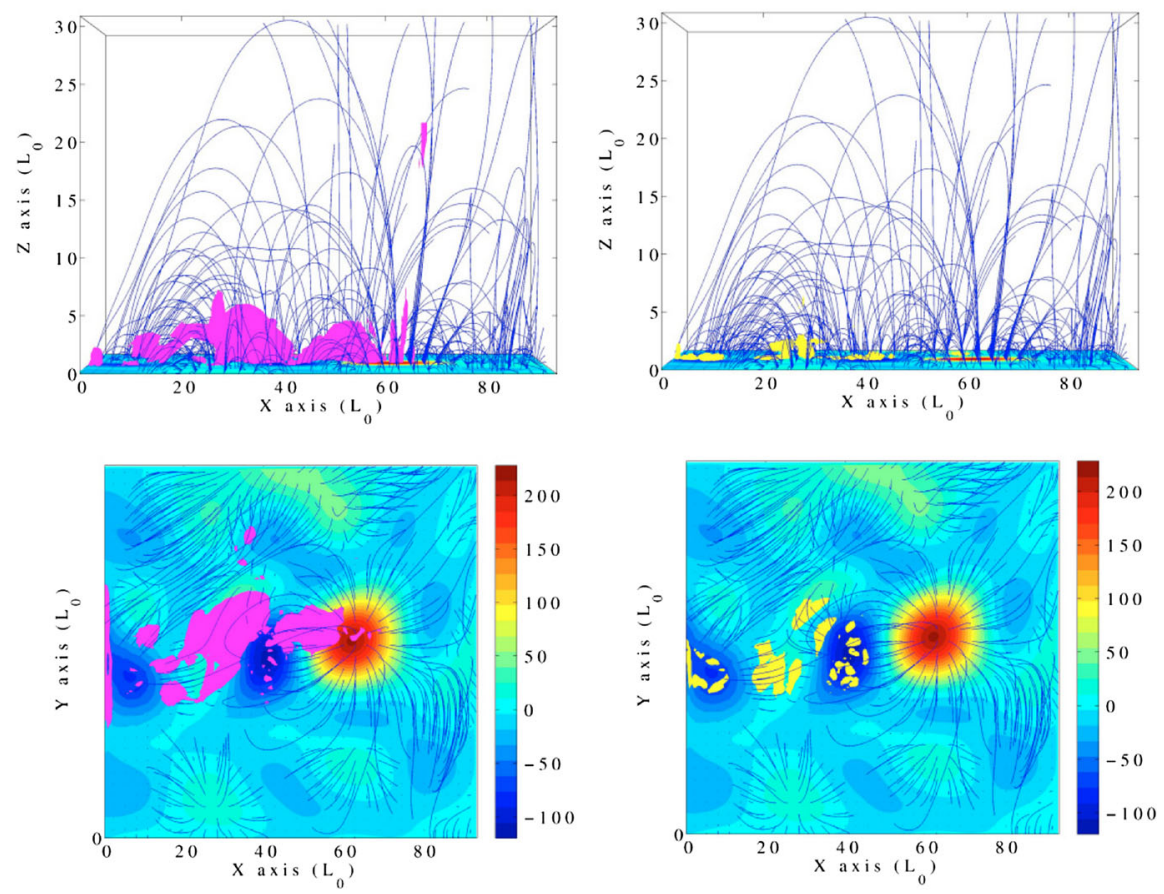

Fig. 40 Lateral (top panels) and top views (bottom panels) of the isosurfaces of a parallel current (left column) and perpendicular current. Velocity pattern shown in the left panel of Fig. 3 in their paper is applied as boundary condition of the model. The isosurfaces of parallel currents are shown in magenta and perpendicular in yellow. Image reproduced with permission from Santos et al. (2008), copyright by ESO

\section{Conclusions and perspectives}

To date, understanding the physical properties of small-scale loops that are heated to coronal temperatures making them visible in EUV and X-rays as bundles of bright loops, has proved challenging but also to a large extent successful. These small-scale loops are omnipresent and are located in the solar atmosphere of the quiet Sun, coronal holes and in the proximity of active regions, and are now generally called coronal bright points or CBPs. Despite of scientific research spanning over five decades, a range of questions remain open including the most crucially important question on how these small-scale loops are heated to coronal temperatures. CBPs are now established to have very similar properties as active regions only at a smaller scale (e.g., temperatures, densities). They appear to be composed of small-scale loops at the different heights and different temperatures that have hotter tops and cooler legs/footpoints, but more evidence from present data is yet to be delivered. The coronal emission increase was found to precede the lower temperature emission suggesting that the heating occurs at coronal heights and is carried to the chromosphere by thermal conduction that can explain both the response in the chromospheric lines and the initial response at coronal temperatures. No recent data were used to confirm this conclusion. Flows are still derived only from Doppler shifts which remains a disputable subject given instrumental 
and line-of-sight effects. Oscillations are interpreted both as propagating magnetoacoustic waves and repetitive reconnection, and no consensus has been reached yet whether they are the signature of both or a unique characteristic of only one of them. It also remains an open question of what photospheric magnetic flux strength relates to CBPs with the last estimation of the flux range dating back to 1979. A unique oneto-one relationship of CBPs and transient phenomena like mass ejections that evolve into collimated flows (jets) in CHs (also near ARs) or loops ejections in the QS is still to be confirmed. A state-of-the-art modelling has shown that the build up of electric currents due to footpoint motions occurs at the location of strong coronal emission in CBPs. However, the time is ripe for the development of a fully data driven MHD model that delivers observables that would be used in forward models to produce synthetic observations which could be compared directly with the original observations to verify the physical mechanisms that generate and sustain CBPs.

There exists a wealth of present observations from SOHO, TRACE, Hinode, STEREO, SDO and IRIS that are still to be explored to investigate important plasma and magnetic properties of CBPs. The Solar Orbiter mission will provide the highest quality of remote sensing measurements which should bring us close to finally obtain a full understanding on the physics of coronal bright points and to verify the exiting theoretical models.

Acknowledgements The author thanks very much Living Reviews in Solar Physics for the invitation to write this review. Many thanks to the anonymous referees for their comments and suggestions. The author is grateful to Klaus Galsgaard for carefully reading and commenting on the theoretical part of the review and also Neil R. Sheeley and Leon Golub for the important suggestions. The author thanks Armin Theissen for carefully reading and commenting on the advanced version of the manuscript. Acknowledgements to the International Space Science Institute, Bern, for the support of the team "Observation-Driven Modelling of Solar Phenomena".

Open Access This article is distributed under the terms of the Creative Commons Attribution 4.0 International License (http://creativecommons.org/licenses/by/4.0/), which permits unrestricted use, distribution, and reproduction in any medium, provided you give appropriate credit to the original author(s) and the source, provide a link to the Creative Commons license, and indicate if changes were made.

\section{References}

Adamson E, Büchner J, Otto A (2013) On the role of current dissipation in the energization of coronal bright points. Astron Astrophys 557:A118. https://doi.org/10.1051/0004-6361/201321281

Alexander CE, Del Zanna G, Maclean RC (2011) Hinode observations and 3D magnetic structure of an X-ray bright point. Astron Astrophys 526:A134. https://doi.org/10.1051/0004-6361/201014045

Alipour N, Safari H (2015) Statistical properties of solar coronal bright points. Astrophys J 807:175. https:// doi.org/10.1088/0004-637X/807/2/175

Bewsher D, Parnell CE, Harrison RA (2002) Transition region blinkers-I. Quiet-Sun properties. Sol Phys 206:21-43. https://doi.org/10.1023/A:1014954629349

Bohlin JD, Vogel SN, Purcell JD, Sheeley NR Jr, Tousey R, Vanhoosier ME (1975) A newly observed solar feature-macrospicules in He II $304 \AA$ A. Astrophys J Lett 197:L133-L135. https://doi.org/10.1086/ 181794

Brajša R, Wöhl H, Vršnak B, Ruždjak V, Clette F, Hochedez JF (2001) Solar differential rotation determined by tracing coronal bright points in SOHO-EIT images. I. Interactive and automatic methods of data reduction. Astron Astrophys 374:309-315. https://doi.org/10.1051/0004-6361:20010694 
Brajša R, Wöhl H, Vršnak B, Ruždjak V, Clette F, Hochedez JF (2002) Solar differential rotation determined by tracing coronal bright points in SOHO-EIT images. II. Results for 1998/99 obtained with interactive and automatic methods. Astron Astrophys 392:329-334. https://doi.org/10.1051/0004-6361: 20020945

Brajša R, Wöhl H, Vršnak B, Ruždjak V, Clette F, Hochedez JF, Roša D (2004) Height correction in the measurement of solar differential rotation determined by coronal bright points. Astron Astrophys 414:707-715. https://doi.org/10.1051/0004-6361:20034082

Brajša R, Wöhl H, Vršnak B, Rušdjak V, Clette F, Hochedez JF, Verbanac G, Temmer M (2005) Spatial distribution and north-south asymmetry of coronal bright points from mid-1998 to mid-1999. Sol Phys 231:29-44. https://doi.org/10.1007/s11207-005-8784-y

Brooks DH, Warren HP (2008) Modeling of the extreme-ultraviolet and soft X-ray emission in a solar coronal bright point. Astrophys J 687:1363-1372. https://doi.org/10.1086/591834

Brosius JW, Rabin DM, Thomas RJ (2007) Doppler velocities measured in coronal emission lines from a bright point observed with the EUNIS sounding rocket. Astrophys J Lett 656:L41-L44. https://doi. org/10.1086/512185

Brosius JW, Rabin DM, Thomas RJ, Landi E (2008) Analysis of a solar coronal bright point extreme ultraviolet spectrum from the EUNIS sounding rocket instrument. Astrophys J 677:781-789. https:// doi.org/10.1086/528930

Brown DS, Parnell CE, Deluca EE, Golub L, McMullen RA (2001) The magnetic structure of a coronal X-ray bright point. Sol Phys 201:305-321. https://doi.org/10.1023/A:1017907406350

Brueckner GE, Bartoe JDF (1983) Observations of high-energy jets in the corona above the quiet Sun, the heating of the corona, and the acceleration of the solar wind. Astrophys J 272:329-348. https://doi. org/10.1086/161297

Büchner J (2006) Locating current sheets in the solar corona. Space Sci Rev 122:149-160. https://doi.org/ 10.1007/s11214-006-8213-z. arXiv:astro-ph/0702460

Chandrashekhar K, Sarkar A (2015) Modeling solar coronal bright-point oscillations with multiple nanoflare heated loops. Astrophys J 810:163. https://doi.org/10.1088/0004-637X/810/2/163. arXiv:1507.04403

Chandrashekhar K, Krishna Prasad S, Banerjee D, Ravindra B, Seaton DB (2013) Dynamics of coronal bright points as seen by Sun Watcher using Active Pixel system detector and image processing (SWAP), Atmospheric Imaging Assembly (AIA), and Helioseismic and Magnetic Imager (HMI). Sol Phys 286:125-142. https://doi.org/10.1007/s11207-012-0046-1. arXiv:1206.0095

Chitta LP, Kariyappa R, van Ballegooijen AA, DeLuca EE, Hasan SS, Hanslmeier A (2013) Observations and modeling of the emerging extreme-ultraviolet loops in the quiet Sun as seen with the Solar Dynamics Observatory. Astrophys J 768:32. https://doi.org/10.1088/0004-637X/768/1/32. arXiv:1303.3426

Christe S, Hannah IG, Krucker S, McTiernan J, Lin RP (2008) RHESSI microflare statistics. I. Flare-finding and frequency distributions. Astrophys J 677:1385-1394. https://doi.org/10.1086/529011

Cirtain JW, Golub L, Lundquist L, van Ballegooijen A, Savcheva A, Shimojo M, DeLuca E, Tsuneta S, Sakao T, Reeves K, Weber M, Kano R, Narukage N, Shibasaki K (2007) Evidence for Alfvén waves in solar X-ray jets. Science 318:1580. https://doi.org/10.1126/science.1147050

Cirtain JW, Golub L, Winebarger AR, de Pontieu B, Kobayashi K, Moore RL, Walsh RW, Korreck KE, Weber M, McCauley P, Title A, Kuzin S, Deforest CE (2013) Energy release in the solar corona from spatially resolved magnetic braids. Nature 493:501-503. https://doi.org/10.1038/nature11772

Culhane JL, Harra LK, James AM, Al-Janabi K, Bradley LJ, Chaudry RA, Rees K, Tandy JA, Thomas P, Whillock MCR, Winter B, Doschek GA, Korendyke CM, Brown CM, Myers S, Mariska J, Seely J, Lang J, Kent BJ, Shaughnessy BM, Young PR, Simnett GM, Castelli CM, Mahmoud S, MapsonMenard H, Probyn BJ, Thomas RJ, Davila J, Dere K, Windt D, Shea J, Hagood R, Moye R, Hara H, Watanabe T, Matsuzaki K, Kosugi T, Hansteen V, Wikstol Ø (2007a) The EUV imaging spectrometer for Hinode. Sol Phys 243:19-61. https://doi.org/10.1007/s01007-007-0293-1

Culhane L, Harra LK, Baker D, van Driel-Gesztelyi L, Sun J, Doschek GA, Brooks DH, Lundquist LL, Kamio S, Young PR, Hansteen VH (2007b) Hinode EUV study of jets in the Sun's south polar corona. Publ Astron Soc Japan 59:S751-S756. https://doi.org/10.1093/pasj/59.sp3.S751

Davis JM (1983) X-ray bright points and the sunspot cycle: further results and predictions. Sol Phys 88:337342. https://doi.org/10.1007/BF00196197

Davis JM, Golub L, Krieger AS (1977) Solar cycle variation of magnetic flux emergence. Astrophys J Lett 214:L141-L144. https://doi.org/10.1086/182461

De Pontieu B, Title AM, Lemen JR, Kushner GD, Akin DJ, Allard B, Berger T, Boerner P, Cheung M, Chou C, Drake JF, Duncan DW, Freeland S, Heyman GF, Hoffman C, Hurlburt NE, Lindgren RW, 
Mathur D, Rehse R, Sabolish D, Seguin R, Schrijver CJ, Tarbell TD, Wülser JP, Wolfson CJ, Yanari C, Mudge J, Nguyen-Phuc N, Timmons R, van Bezooijen R, Weingrod I, Brookner R, Butcher G, Dougherty B, Eder J, Knagenhjelm V, Larsen S, Mansir D, Phan L, Boyle P, Cheimets PN, DeLuca EE, Golub L, Gates R, Hertz E, McKillop S, Park S, Perry T, Podgorski WA, Reeves K, Saar S, Testa P, Tian H, Weber M, Dunn C, Eccles S, Jaeggli SA, Kankelborg CC, Mashburn K, Pust N, Springer L, Carvalho R, Kleint L, Marmie J, Mazmanian E, Pereira TMD, Sawyer S, Strong J, Worden SP, Carlsson M, Hansteen VH, Leenaarts J, Wiesmann M, Aloise J, Chu KC, Bush RI, Scherrer PH, Brekke P, Martinez-Sykora J, Lites BW, McIntosh SW, Uitenbroek H, Okamoto TJ, Gummin MA, Auker G, Jerram P, Pool P, Waltham N (2014) The Interface Region Imaging Spectrograph (IRIS). Sol Phys 289:2733-2779. https://doi.org/10.1007/s11207-014-0485-y. arXiv:1401.2491

Del Zanna G, Mason HE (2003) Solar active regions: SOHO/CDS and TRACE observations of quiescent coronal loops. Astron Astrophys 406:1089-1103. https://doi.org/10.1051/0004-6361:20030791

Del Zanna G, Bromage BJI, Mason HE (2003) Spectroscopic characteristics of polar plumes. Astron Astrophys 398:743-761. https://doi.org/10.1051/0004-6361:20021628

Delaboudinière JP, Artzner GE, Brunaud J, Gabriel AH, Hochedez JF, Millier F, Song XY, Au B, Dere KP, Howard RA, Kreplin R, Michels DJ, Moses JD, Defise JM, Jamar C, Rochus P, Chauvineau JP, Marioge JP, Catura RC, Lemen JR, Shing L, Stern RA, Gurman JB, Neupert WM, Maucherat A, Clette F, Cugnon P, van Dessel EL (1995) EIT: extreme-ultraviolet imaging telescope for the SOHO mission. Sol Phys 162:291-312. https://doi.org/10.1007/BF00733432

Dere KP (2008) The plasma filling factor of coronal bright points. Coronal bright points. Astron Astrophys 491:561-566. https://doi.org/10.1051/0004-6361:200810000

Dere KP (2009) The plasma filling factor of coronal bright points. II. Combined EIS and TRACE results. Astron Astrophys 497:287-290. https://doi.org/10.1051/0004-6361/200811329

Dodson HW (1953) High latitude sunspot, August 13, 1953. Publ Astron Soc Pac 65:256. https://doi.org/ $10.1086 / 126615$

Domingo V, Fleck B, Poland AI (1995) The SOHO mission: an overview. Sol Phys 162:1-37. https://doi. org/10.1007/BF00733425

Doschek GA, Landi E, Warren HP, Harra LK (2010) Bright points and jets in polar coronal holes observed by the extreme-ultraviolet imaging spectrometer on Hinode. Astrophys J 710:1806-1824. https://doi. org/10.1088/0004-637X/710/2/1806

Driesman A, Hynes S, Cancro G (2008) The STEREO Observatory. Space Sci Rev 136:17-44. https://doi. org/10.1007/s11214-007-9286-Z

Feldman U, Mandelbaum P, Seely JF, Doschek GA, Gursky H (1992) The potential for plasma diagnostics from stellar extreme-ultraviolet observations. Astrophys J Suppl Ser 81:387-408. https://doi.org/10. $1086 / 191698$

Fu Q, Kundu MR, Schmahl EJ (1987) Coronal bright points at $6 \mathrm{~cm}$ wavelength. Sol Phys 108:99-111. https://doi.org/10.1007/BF00152080

Galsgaard K, Parnell CE (2005) Elementary heating events-magnetic interactions between two flux sources. III. Energy considerations. Astron Astrophys 439:335-349. https://doi.org/10.1051/00046361:20052775. arXiv:astro-ph/0501602

Galsgaard K, Parnell CE, Blaizot J (2000) Elementary heating events-magnetic interactions between two flux sources. Astron Astrophys 362:395-405

Galsgaard K, Madjarska MS, Moreno-Insertis F, Huang Z, Wiegelmann T (2017) Magnetic topological analysis of coronal bright points. Astron Astrophys 606:A46. https://doi.org/10.1051/0004-6361/ 201731041. arXiv:1707.04174

Giovanelli RG (1982) On the relative roles of unipolar and mixed-polarity fields. Sol Phys 77:27-42. https:// doi.org/10.1007/BF00156093

Glencross WM (1975) Heating of coronal material at X-ray bright points. Astrophys J Lett 199:L53-L56. https://doi.org/10.1086/181847

Golub L, Krieger AS, Silk JK, Timothy AF, Vaiana GS (1974) Solar X-ray bright points. Astrophys J Lett 189:L93. https://doi.org/10.1086/181472

Golub L, Krieger AS, Vaiana GS (1975) Observation of a non-uniform component in the distribution of coronal bright points. Sol Phys 42:131-134. https://doi.org/10.1007/BF00153290

Golub L, Krieger AS, Vaiana GS (1976a) Distribution of lifetimes for coronal soft X-ray bright points. Sol Phys 49:79-90. https://doi.org/10.1007/BF00221486

Golub L, Krieger AS, Vaiana GS (1976b) Observation of spatial and temporal variations in X-ray bright point emergence patterns. Sol Phys 50:311-327. https://doi.org/10.1007/BF00155294 
Golub L, Krieger AS, Harvey JW, Vaiana GS (1977) Magnetic properties of X-ray bright points. Sol Phys 53:111-121. https://doi.org/10.1007/BF02260212

Golub L, Davis JM, Krieger AS (1979) Anticorrelation of X-ray bright points with sunspot number, 19701978. Astrophys J Lett 229:L145-L150. https://doi.org/10.1086/182949

Golub L, Deluca E, Austin G, Bookbinder J, Caldwell D, Cheimets P, Cirtain J, Cosmo M, Reid P, Sette A, Weber M, Sakao T, Kano R, Shibasaki K, Hara H, Tsuneta S, Kumagai K, Tamura T, Shimojo M, McCracken J, Carpenter J, Haight H, Siler R, Wright E, Tucker J, Rutledge H, Barbera M, Peres G, Varisco S (2007) The X-Ray Telescope (XRT) for the Hinode mission. Sol Phys 243:63-86. https:// doi.org/10.1007/s11207-007-0182-1

Grevesse N, Sauval AJ (1998) Standard solar composition. Space Sci Rev 85:161-174. https://doi.org/10. 1023/A:1005161325181

Guglielmino SL, Martínez Pillet V, Bonet JA, del Toro Iniesta JC, Bellot Rubio LR, Solanki SK, Schmidt W, Gandorfer A, Barthol P, Knölker M (2012) The frontier between small-scale bipoles and ephemeral regions in the solar photosphere: emergence and decay of an intermediate-scale bipole observed with SUNRISE/IMaX. Astrophys J 745:160. https://doi.org/10.1088/0004-637X/745/2/160. arXiv: 1110.1405

Habbal SR, Grace E (1991) The connection between coronal bright points and the variability of the quiet-Sun extreme-ultraviolet emission. Astrophys J 382:667-676. https://doi.org/10.1086/170754

Habbal SR, Harvey KL (1988) Simultaneous observations of 20 centimeter bright points and He I 10830 $\AA$ dark points in the quiet Sun. Astrophys J 326:988-996. https://doi.org/10.1086/166156

Habbal SR, Withbroe GL (1981) Spatial and temporal variations of EUV coronal bright points. Sol Phys 69:77-97. https://doi.org/10.1007/BF00151257

Habbal SR, Ronan RS, Withbroe GL, Shevgaonkar RK, Kundu MR (1986) Solar coronal bright points observed with the VLA. Astrophys J 306:740-750. https://doi.org/10.1086/164383

Habbal SR, Withbroe GL, Dowdy JF Jr (1990) A comparison between bright points in a coronal hole and a quiet-Sun region. Astrophys J 352:333-342. https://doi.org/10.1086/168540

Hachenberg O, Steffen P, Harth W (1978) The Sun at $8.5 \mathrm{~mm}$ wavelength-results of observations with high angular resolution. Sol Phys 60:105-118. https://doi.org/10.1007/BF00152335

Handy BN, Acton LW, Kankelborg CC, Wolfson CJ, Akin DJ, Bruner ME, Caravalho R, Catura RC, Chevalier R, Duncan DW, Edwards CG, Feinstein CN, Freeland SL, Friedlaender FM, Hoffmann CH, Hurlburt NE, Jurcevich BK, Katz NL, Kelly GA, Lemen JR, Levay M, Lindgren RW, Mathur DP, Meyer SB, Morrison SJ, Morrison MD, Nightingale RW, Pope TP, Rehse RA, Schrijver CJ, Shine RA, Shing L, Strong KT, Tarbell TD, Title AM, Torgerson DD, Golub L, Bookbinder JA, Caldwell D, Cheimets PN, Davis WN, Deluca EE, McMullen RA, Warren HP, Amato D, Fisher R, Maldonado H, Parkinson C (1999) The Transition Region and Coronal Explorer. Sol Phys 187:229-260. https:// doi.org/10.1023/A:1005166902804

Hara H, Nakakubo-Morimoto K (2003) Variation of the X-ray bright point number over the solar activity cycle. Astrophys J 589:1062-1074. https://doi.org/10.1086/374778

Harrison RA (1997) EUV blinkers: the significance of variations in the extreme ultraviolet quiet Sun. Sol Phys 175:467-485. https://doi.org/10.1023/A:1004964707047

Harrison RA, Sawyer EC, Carter MK, Cruise AM, Cutler RM, Fludra A, Hayes RW, Kent BJ, Lang J, Parker DJ, Payne J, Pike CD, Peskett SC, Richards AG, Gulhane JL, Norman K, Breeveld AA, Breeveld ER, Al Janabi KF, McCalden AJ, Parkinson JH, Self DG, Thomas PD, Poland AI, Thomas RJ, Thompson WT, Kjeldseth-Moe O, Brekke P, Karud J, Maltby P, Aschenbach B, Bräuninger H, Kühne M, Hollandt J, Siegmund OHW, Huber MCE, Gabriel AH, Mason HE, Bromage BJI (1995) The coronal diagnostic spectrometer for the Solar and Heliospheric Observatory. Sol Phys 162:233-290. https://doi.org/10. 1007/BF00733431

Harrison RA, Harra LK, Brković A, Parnell CE (2003) A study of the unification of quiet-Sun transient-event phenomena. Astron Astrophys 409:755-764. https://doi.org/10.1051/0004-6361:20031072

Harvey KL (1984) Solar cycle variation of ephemeral active regions. In: Guyenne TD, Hunt JJ (eds) The hydromagnetics of the Sun, ESA special publication, vol 220. ESA, Paris, p 235

Harvey KL (1985) The relationship between coronal bright points as seen in He I $\lambda 10830$ and the evolution of the photospheric network magnetic fields. Aust J Phys 38:875-883. https://doi.org/10.1071/PH850875

Harvey KL, Martin SF (1973) Ephemeral active regions. Sol Phys 32:389-402. https://doi.org/10.1007/ BF00154951

Harvey KL, Harvey JW, Martin SF (1975) Ephemeral active regions in 1970 and 1973. Sol Phys 40:87-102. https://doi.org/10.1007/BF00183154 
Harvey KL, Strong KT, Nitta N, Tsuneta S (1993) Lifetimes and distribution of coronal bright points observed with Yohkoh. Adv Space Res 13:27-30. https://doi.org/10.1016/0273-1177(93)90453-I

Harvey KL, Strong KS, Nitta N, Tsuneta S (1994) Are X-ray bright points the signature of magnetic field reconnection? In: Balasubramaniam KS, Simon GW (eds) Solar active region evolution: comparing models with observations, ASP conference series, vol 68. Astronomical Society of the Pacific, San Francisco, p 377

Hermans LM, Martin SF (1986) Small-scale eruptive filaments on the quiet Sun. In: Poland AI (ed) Coronal and prominence plasmas, NASA conference publication, vol 2442. NASA, p 369

Hirzberger J, Gizon L, Solanki SK, Duvall TL (2008) Structure and evolution of supergranulation from local helioseismology. Sol Phys 251:417-437. https://doi.org/10.1007/s11207-008-9206-8

Hong J, Jiang Y, Yang J, Bi Y, Li H, Yang B, Yang D (2014) Coronal bright points associated with minifilament eruptions. Astrophys J 796:73. https://doi.org/10.1088/0004-637X/796/2/73

Innes DE, Genetelli A, Attie R, Potts HE (2009) Quiet Sun mini-coronal mass ejections activated by supergranular flows. Astron Astrophys 495:319-323. https://doi.org/10.1051/0004-6361:200811011. arXiv:0811.2744

Innes DE, McIntosh SW, Pietarila A (2010) STEREO quadrature observations of coronal dimming at the onset of mini-CMEs. Astron Astrophys 517:L7. https://doi.org/10.1051/0004-6361/201014366. arXiv: 1005.2097

Janssen MA, Olsen ET, Lang KR (1979) Interferometric observations of the quiet Sun at 8 millimeter wavelength. Astrophys J 228:616-623. https://doi.org/10.1086/156886

Javadi S, Büchner J, Otto A, Santos JC (2011) About the relative importance of compressional heating and current dissipation for the formation of coronal X-ray bright points. Astron Astrophys 529:A114. https://doi.org/10.1051/0004-6361/201015614. arXiv:1012.0761

Jordan C, Ayres TR, Brown A, Linsky JL, Simon T (1987) The chromospheres and coronae of five G-K main-sequence stars. Mon Not R Astron Soc 225:903-937. https://doi.org/10.1093/mnras/225.4.903

Kaiser ML, Kucera TA, Davila JM, St Cyr OC, Guhathakurta M, Christian E (2008) The STEREO mission: an introduction. Space Sci Rev 136:5-16. https://doi.org/10.1007/s11214-007-9277-0

Kamio S, Curdt W, Teriaca L, Innes DE (2011) Evolution of microflares associated with bright points in coronal holes and in quiet regions. Astron Astrophys 529:A21. https://doi.org/10.1051/0004-6361/ 201015715. arXiv:1009.1957

Kankelborg C, Longcope D (1999) Forward modeling of the coronal response to reconnection in an X-ray bright point. Sol Phys 190:59-77. https://doi.org/10.1023/A:1005205807984

Kankelborg CC, Walker ABC II, Hoover RB, Barbee TW Jr (1996) Observation and modeling of soft X-ray bright points. I. Initial results. Astrophys J 466:529. https://doi.org/10.1086/177529

Kankelborg CC, Walker ABC II, Hoover RB (1997) Observation and modeling of soft X-ray bright points. II. Determination of temperature and energy balance. Astrophys J 491:952-966. https://doi.org/10. $1086 / 304976$

Kariyappa R, Varghese BA (2008) Intensity oscillations and heating of the coronal X-ray bright points from Hinode/XRT. Astron Astrophys 485:289-292. https://doi.org/10.1051/0004-6361:20079127. arXiv:0804.3480

Kariyappa R, Deluca EE, Saar SH, Golub L, Damé L, Pevtsov AA, Varghese BA (2011) Temperature variability in X-ray bright points observed with Hinode/XRT. Astron Astrophys 526:A78. https://doi. org/10.1051/0004-6361/201014878

Kayshap P, Dwivedi BN (2017) Diagnostics of coronal bright points using IRIS, AIA, and HMI observations. Sol Phys 292:108. https://doi.org/10.1007/s11207-017-1132-1

Klimchuk JA, Patsourakos S, Cargill PJ (2008) Highly efficient modeling of dynamic coronal loops. Astrophys J 682:1351-1362. https://doi.org/10.1086/589426. arXiv:0710.0185

Kosugi T, Matsuzaki K, Sakao T, Shimizu T, Sone Y, Tachikawa S, Hashimoto T, Minesugi K, Ohnishi A, Yamada T, Tsuneta S, Hara H, Ichimoto K, Suematsu Y, Shimojo M, Watanabe T, Shimada S, Davis JM, Hill LD, Owens JK, Title AM, Culhane JL, Harra LK, Doschek GA, Golub L (2007) The Hinode (Solar-B) mission: an overview. Sol Phys 243:3-17. https://doi.org/10.1007/s11207-007-9014-6

Krieger AS, Vaiana GS, van Speybroeck LP (1971) The X-ray corona and the photospheric magnetic field. In: Howard R (ed) Solar magnetic fields, D. Reidel, Dordrecht. IAU symposium, vol 43, pp 397-412. https://doi.org/10.1007/978-94-010-3117-2_52

Kumar M, Srivastava AK, Dwivedi BN (2011) Observation of intensity oscillations above X-ray bright points from the Hinode/XRT: signature of magnetohydrodynamic oscillations in the solar corona. Mon Not R Astron Soc 415:1419-1425. https://doi.org/10.1111/j.1365-2966.2011.18792.x 
Kundu MR, Gergely TE, Golub L (1980) Association of flaring X-ray bright points with type III bursts. Astrophys J Lett 236:L87-L90. https://doi.org/10.1086/183204

Kundu MR, Schmahl EJ, Fu QJ (1988) Coronal bright points at 6 and 20 centimeter wavelengths. Astrophys J 325:905-911. https://doi.org/10.1086/166062

Kundu MR, Shibasaki K, Enome S, Nitta N (1994a) Detection of $17 \mathrm{GHz}$ radio emission from X-ray-bright points. Astrophys J Lett 431:L155-L158. https://doi.org/10.1086/187496

Kundu MR, Strong KT, Pick M, White SM, Hudson HS, Harvey KL, Kane SR (1994b) Nonthermal processes in flaring X-ray-bright points. Astrophys J Lett 427:L59-L62. https://doi.org/10.1086/187364

Kundu MR, Raulin JP, Pick M, Strong KT (1995) Metric type III bursts from a flaring X-ray bright point near an active region. Astrophys J 444:922-928. https://doi.org/10.1086/175662

Kwon RY, Chae J, Zhang J (2010) Stereoscopic determination of heights of extreme ultraviolet bright points using data taken by SECCHI/EUVI aboard STEREO. Astrophys J 714:130-137. https://doi.org/10. 1088/0004-637X/714/1/130

Kwon RY, Chae J, Davila JM, Zhang J, Moon YJ, Poomvises W, Jones SI (2012) Three-dimensional structure and evolution of extreme-ultraviolet bright points observed by STEREO/SECCHI/EUVI. Astrophys J 757:167. https://doi.org/10.1088/0004-637X/757/2/167

Lee KS, Brooks DH, Imada S (2015) Photospheric abundances of polar jets on the Sun observed by Hinode. Astrophys J 809:114. https://doi.org/10.1088/0004-637X/809/2/114. arXiv:1507.04075

Lemaire P, Wilhelm K, Curdt W, Schule U, Marsch E, Poland AI, Jordan SD, Thomas RJ, Hassler DM, Vial JC, Kuhne M, Huber MCE, Siegmund OHW, Gabriel A, Timothy JG, Grewing M (1997) First results of the SUMER telescope and spectrometer on SOHO-II. Imagery and data management. Sol Phys 170:105-122. https://doi.org/10.1023/A:1004994702333

Lemen JR, Title AM, Akin DJ, Boerner PF, Chou C, Drake JF, Duncan DW, Edwards CG, Friedlaender FM, Heyman GF, Hurlburt NE, Katz NL, Kushner GD, Levay M, Lindgren RW, Mathur DP, McFeaters EL, Mitchell S, Rehse RA, Schrijver CJ, Springer LA, Stern RA, Tarbell TD, Wuelser JP, Wolfson CJ, Yanari C, Bookbinder JA, Cheimets PN, Caldwell D, Deluca EE, Gates R, Golub L, Park S, Podgorski WA, Bush RI, Scherrer PH, Gummin MA, Smith P, Auker G, Jerram P, Pool P, Soufli R, Windt DL, Beardsley S, Clapp M, Lang J, Waltham N (2012) The Atmospheric Imaging Assembly (AIA) on the Solar Dynamics Observatory (SDO). Sol Phys 275:17-40. https://doi.org/10.1007/s11207-0119776-8

Li D, Ning Z, Su Y (2016) The bi-directional moving structures in a coronal bright point. Astrophys Space Sci 361:301. https://doi.org/10.1007/s10509-016-2893-y. arXiv:1608.01534

Longcope DW (1996) Topology and current ribbons: a model for current, reconnection and flaring in a complex, evolving corona. Sol Phys 169:91-121. https://doi.org/10.1007/BF00153836

Longcope DW (1998) A model for current sheets and reconnection in X-ray bright points. Astrophys J 507:433-442. https://doi.org/10.1086/306319

Longcope DW, Kankelborg CC, Nelson JL, Pevtsov AA (2001) Evidence of separator reconnection in a survey of X-ray bright points. Astrophys J 553:429-439. https://doi.org/10.1086/320667

Madjarska MS (2011) Dynamics and plasma properties of an X-ray jet from SUMER, EIS, XRT, and EUVI A \& B simultaneous observations. Astron Astrophys 526:A19. https://doi.org/10.1051/0004-6361/ 201015269. arXiv: 1008.3983

Madjarska MS, Doyle JG, Teriaca L, Banerjee D (2003) An EUV bright point as seen by SUMER, CDS, MDI and EIT on-board SoHO. Astron Astrophys 398:775-784. https://doi.org/10.1051/0004-6361: 20021732

Madjarska MS, Huang Z, Doyle JG, Subramanian S (2012) Coronal hole boundaries evolution at small scales. III. EIS and SUMER views. Astron Astrophys 545:A67. https://doi.org/10.1051/0004-6361/ 201219516. arXiv: 1207.1281

Mandrini CH, Démoulin P, van Driel-Gesztelyi L, Schmieder B, Cauzzi G, Hofmann A (1996) 3D magnetic reconnection at an X-ray bright point. Sol Phys 168:115-133. https://doi.org/10.1007/BF00145829

Mandrini CH, Pohjolainen S, Dasso S, Green LM, Démoulin P, van Driel-Gesztelyi L, Copperwheat C, Foley C (2005) Interplanetary flux rope ejected from an X-ray bright point. The smallest magnetic cloud source-region ever observed. Astron Astrophys 434:725-740. https://doi.org/10.1051/00046361:20041079

Marsh KA, Hurford GJ, Zirin H (1980) VLA observations of spatial structure in the quiet Sun at 6 centimeters, during the 1977 October eclipse. Astrophys J 236:1017-1025. https://doi.org/10.1086/157828 
Martens PCH, Cirtain JW, Schmelz JT (2002) The inadequacy of temperature measurements in the solar corona through narrowband filter and line ratios. Astrophys J Lett 577:L115-L117. https://doi.org/10. $1086 / 344254$

McIntosh SW (2007) On the mass and energy loading of extreme-UV bright points. Astrophys J 670:14011413. https://doi.org/10.1086/521948. arXiv:0708.0550

McIntosh SW, Gurman JB (2005) Nine years of EUV bright points. Sol Phys 228:285-299. https://doi.org/ $10.1007 / \mathrm{s} 11207-005-4725-\mathrm{z}$

McIntosh SW, Wang X, Leamon RJ, Davey AR, Howe R, Krista LD, Malanushenko AV, Markel RS, Cirtain JW, Gurman JB, Pesnell WD, Thompson MJ (2014a) Deciphering solar magnetic activity. I. On the relationship between the sunspot cycle and the evolution of small magnetic features. Astrophys $\mathrm{J}$ 792:12. https://doi.org/10.1088/0004-637X/792/1/12. arXiv:1403.3071

McIntosh SW, Wang X, Leamon RJ, Scherrer PH (2014b) Identifying potential markers of the Sun's giant convective scale. Astrophys J Lett 784:L32. https://doi.org/10.1088/2041-8205/784/2/L32. arXiv:1403.0692

Moore RL, Tang F, Bohlin JD, Golub L (1977) H $\alpha$ macrospicules: identification with EUV macrospicules and with flares in X-ray bright points. Astrophys J 218:286-290. https://doi.org/10.1086/155681

Moses D, Cook JW, Bartoe JDF, Brueckner GE, Dere KP, Webb DF, Davis JM, Harvey JW, Recely F, Martin SF, Zirin H (1994) Solar fine scale structures in the corona, transition region, and lower atmosphere. Astrophys J 430:913-924. https://doi.org/10.1086/174461

Mou C, Huang Z, Xia L, Madjarska MS, Li B, Fu H, Jiao F, Hou Z (2016) Magnetic flux supplement to coronal bright points. Astrophys J 818:9. https://doi.org/10.3847/0004-637X/818/1/9. arXiv:1511.09215

Mou C, Madjarska MS, Galsgaard K, Xia L (2018) Eruptions from quiet Sun coronal bright points. I. Observations. Astron Astrophys 619:A55. https://doi.org/10.1051/0004-6361/201833243. arXiv: 1808.04541

Nakakubo K, Hara H (2000) Variation of X-ray bright point number over the solar activity cycle. Adv Space Res 25:1905-1908. https://doi.org/10.1016/S0273-1177(99)00621-3

Ning Z, Guo Y (2014) Investigation of the moving structures in a coronal bright point. Astrophys J 794:79. https://doi.org/10.1088/0004-637X/794/1/79

Nitta N, Kundu MR (1988) A study of coronal bright points at $20 \mathrm{~cm}$ wavelength. Sol Phys 117:37-50. https://doi.org/10.1007/BF00148570

Nitta N, Bastian TS, Aschwanden MJ, Harvey KL, Strong KT (1992) Simultaneous observations of coronal bright points in X-ray and radio wavelengths. Publ Astron Soc Japan 44:L167-L172

Nolte JT, Solodyna CV, Gerassimenko M (1979) Short-term temporal variations of X-ray bright points. Sol Phys 63:113-118. https://doi.org/10.1007/BF00155701

Orange NB, Oluseyi HM, Chesny DL, Patel M, Hesterly K, Preuss L, Neira C, Turner NE (2014) Comparative analysis of a transition region bright point with a blinker and coronal bright point using multiple EIS emission lines. Sol Phys 289:1557-1584. https://doi.org/10.1007/s11207-013-0423-4. arXiv: 1501.04997

Paraschiv AR, Lacatus DA, Badescu T, Lupu MG, Simon S, Sandu SG, Mierla M, Rusu MV (2010) Study of coronal jets during solar minimum based on STEREO/SECCHI observations. Sol Phys 264:365-375. https://doi.org/10.1007/s11207-010-9584-6

Parker EN (1975) X-ray bright spots on the Sun and the nonequilibrium of a twisted flux rope in a stratified atmosphere. Astrophys J 201:494-501. https://doi.org/10.1086/153911

Parnell CE, Galsgaard K (2004) Elementary heating events-magnetic interactions between two flux sources. II. Rates of flux reconnection. Astron Astrophys 428:595-612. https://doi.org/10.1051/00046361:20034350

Parnell CE, Priest ER, Golub L (1994a) The three-dimensional structures of X-ray bright points. Sol Phys 151:57-74. https://doi.org/10.1007/BF00654082

Parnell CE, Priest ER, Titov VS (1994b) A model for X-ray bright points due to unequal cancelling flux sources. Sol Phys 153:217-235. https://doi.org/10.1007/BF00712503

Pérez-Suárez D, Maclean RC, Doyle JG, Madjarska MS (2008) The structure and dynamics of a bright point as seen with Hinode, SoHO and TRACE. Astron Astrophys 492:575-583. https://doi.org/10. 1051/0004-6361:200809507. arXiv:0810.1020

Pesnell WD, Thompson BJ, Chamberlin PC (2012) The Solar Dynamics Observatory (SDO). Sol Phys 275:3-15. https://doi.org/10.1007/s11207-011-9841-3 
Podladchikova O, Vourlidas A, Van der Linden RAM, Wülser JP, Patsourakos S (2010) Extreme ultraviolet observations and analysis of micro-eruptions and their associated coronal waves. Astrophys J 709:369376. https://doi.org/10.1088/0004-637X/709/1/369

Popescu MD, Doyle JG, Xia LD (2004) Network boundary origins of fast solar wind seen in the low transition region? Astron Astrophys 421:339-348. https://doi.org/10.1051/0004-6361:20034348

Preś P, Phillips KHJ (1999) The magnetic association of coronal bright points. Astrophys J Lett 510:L73L76. https://doi.org/10.1086/311791. arXiv:astro-ph/9810451

Priest ER, Démoulin P (1995) Three-dimensional magnetic reconnection without null points. 1. Basic theory of magnetic flipping. J Geophys Res 100:23443-23464. https://doi.org/10.1029/95JA02740

Priest ER, Parnell CE, Martin SF (1994) A converging flux model of an X-ray bright point and an associated canceling magnetic feature. Astrophys J 427:459-474. https://doi.org/10.1086/174157

Priest ER, Longcope DW, Titov VS (2003) Binary reconnection and the heating of the solar corona. Astrophys J 598:667-677. https://doi.org/10.1086/378768

Raouafi NE, Patsourakos S, Pariat E, Young PR, Sterling AC, Savcheva A, Shimojo M, Moreno-Insertis F, DeVore CR, Archontis V, Török T, Mason H, Curdt W, Meyer K, Dalmasse K, Matsui Y (2016) Solar coronal jets: observations, theory, and modeling. Space Sci Rev 201:1-53. https://doi.org/10.1007/ s11214-016-0260-5. arXiv:1607.02108

Reale F (2014) Coronal loops: observations and modeling of confined plasma. Living Rev Sol Phys 11:4. https://doi.org/10.12942/lrsp-2014-4

Rosa D, Vrsnak B, Bozic H, Brajsa R, Ruzdjak V, Schroll A, Wohl H (1998) A method to determine the solar synodic rotation rate and the height of tracers. Sol Phys 179:237-252. https://doi.org/10.1023/ A: 1005035213782

Rovira M, Schmieder B, Démoulin P, Simnett GM, Hagyard MJ, Reichmann E, Tandberg-Hanssen E (1999) Bright points and subflares in ultraviolet lines and X-rays. Astrophys J 510:474-484. https://doi.org/ $10.1086 / 306566$

Samanta T, Banerjee D, Tian H (2015) Quasi-periodic oscillation of a coronal bright point. Astrophys J 806:172. https://doi.org/10.1088/0004-637X/806/2/172. arXiv:1505.00587

Santos JC, Büchner J (2007) MHD simulation of electric currents in the solar atmosphere caused by photospheric plasma motion. Astrophys Space Sci Trans 3:29-33. https://doi.org/10.5194/astra-329-2007

Santos JC, Büchner J, Madjarska MS, Alves MV (2008) On the relation between DC current locations and an EUV bright point: a case study. Astron Astrophys 490:345-352. https://doi.org/10.1051/00046361:200809975

Sattarov I, Pevtsov AA, Hojaev AS, Sherdonov CT (2002) X-ray bright points and photospheric bipoles during cycles 22 and 23. Astrophys J 564:1042-1047. https://doi.org/10.1086/324306

Sattarov I, Pevtsov AA, Karachik NV, Sherdanov CT, Tillaboev AM (2010) Solar cycle 23 in coronal bright points. Sol Phys 262:321-335. https://doi.org/10.1007/s11207-010-9524-5

Scherrer PH, Schou J, Bush RI, Kosovichev AG, Bogart RS, Hoeksema JT, Liu Y, Duvall TL, Zhao J, Title AM, Schrijver CJ, Tarbell TD, Tomczyk S (2012) The Helioseismic and Magnetic Imager (HMI) Investigation for the Solar Dynamics Observatory (SDO). Sol Phys 275:207-227. https://doi.org/10. 1007/s11207-011-9834-2

Schmelz JT, Winebarger AR, Kimble JA, Pathak S, Golub L, Jenkins BS, Worley BT (2013) Bright points: multithermal analysis as a test of steady heating models. Astrophys J 770:160. https://doi.org/10.1088/ 0004-637X/770/2/160

Sheeley NR Jr (1995) A volcanic origin for high-FIP material in the solar atmosphere. Astrophys J 440:884. https://doi.org/10.1086/175326

Sheeley NR Jr (1996) Elemental abundance variations in the solar atmosphere. Astrophys J 469:423. https:// doi.org/10.1086/177792

Sheeley NR Jr, Golub L (1979) Rapid changes in the fine structure of a coronal 'bright point' and a small coronal 'active region'. Sol Phys 63:119-126. https://doi.org/10.1007/BF00155702

Shibata K, Ishido Y, Acton LW, Strong KT, Hirayama T, Uchida Y, McAllister AH, Matsumoto R, Tsuneta S, Shimizu T, Hara H, Sakurai T, Ichimoto K, Nishino Y, Ogawara Y (1992) Observations of X-ray jets with the Yohkoh Soft X-ray Telescope. Publ Astron Soc Japan 44:L173-L179

Shimojo M, Shibata K (1999) Occurrence rate of microflares in an X-ray-bright point within an active region. Astrophys J 516:934-938. https://doi.org/10.1086/307156

Simon GW, Noyes RW (1972) Observed heights of EUV lines formed in the transition zone and corona. Sol Phys 22:450-458. https://doi.org/10.1007/BF00148710 
Strong KT, Harvey K, Hirayama T, Nitta N, Shimizu T, Tsuneta S (1992) Observations of the variability of coronal bright points by the Soft X-ray Telescope on Yohkoh. Publ Astron Soc Japan 44:L161-L166

Subramanian S, Madjarska MS, Doyle JG (2010) Coronal hole boundaries evolution at small scales. II. XRT view. Can small-scale outflows at $\mathrm{CHBs}$ be a source of the slow solar wind. Astron Astrophys 516:A50. https://doi.org/10.1051/0004-6361/200913624. arXiv:1002.1675

Subramanian S, Madjarska MS, Doyle JG, Bewsher D (2012) What is the true nature of blinkers? Astron Astrophys 538:A50. https://doi.org/10.1051/0004-6361/201117877. arXiv:1111.2149

Sudar D, Saar SH, Skokić I, Poljančić Beljan I, Brajša R (2016) Meridional motions and Reynolds stress from SDO/AIA coronal bright points data. Astron Astrophys 587:A29. https://doi.org/10.1051/00046361/201527217. arXiv:1601.02406

Tang F, Harvey K, Bruner M, Kent B, Antonucci E (1982) Bright point study. Adv Space Res 2:65-72. https://doi.org/10.1016/0273-1177(82)90181-8

Thompson AR, Clark BG, Wade CM, Napier PJ (1980) The Very Large Array. Astrophys J Suppl 44:151167. https://doi.org/10.1086/190688

Tian H, Tu CY, He JS, Marsch E (2007) Emission heights of coronal bright points on Fe XII radiance map. Adv Space Res 39:1853-1859. https://doi.org/10.1016/j.asr.2007.03.065. arXiv:0907.4954

Tian H, Curdt W, Marsch E, He J (2008a) Cool and hot components of a coronal bright point. Astrophys J Lett 681:L121. https://doi.org/10.1086/590410. arXiv:0906.3005

Tian H, Xia LD, Li S (2008b) Long-period oscillations in solar coronal bright points. Astron Astrophys 489:741-745. https://doi.org/10.1051/0004-6361:200810146

Tian H, Marsch E, Curdt W, He J (2009) Upflows in funnel-like legs of coronal magnetic loops. Astrophys J 704:883-890. https://doi.org/10.1088/0004-637X/704/1/883. arXiv:0909.0739

Tsuneta S, Acton L, Bruner M, Lemen J, Brown W, Caravalho R, Catura R, Freeland S, Jurcevich B, Morrison M, Ogawara Y, Hirayama T, Owens J (1991) The Soft X-ray Telescope for the Solar-A Mission. Sol Phys 136:37-67. https://doi.org/10.1007/BF00151694

Tsuneta S, Ichimoto K, Katsukawa Y, Nagata S, Otsubo M, Shimizu T, Suematsu Y, Nakagiri M, Noguchi M, Tarbell T, Title A, Shine R, Rosenberg W, Hoffmann C, Jurcevich B, Kushner G, Levay M, Lites B, Elmore D, Matsushita T, Kawaguchi N, Saito H, Mikami I, Hill LD, Owens JK (2008) The Solar Optical Telescope for the Hinode mission: an overview. Sol Phys 249:167-196. https://doi.org/10. 1007/s11207-008-9174-z. arXiv:0711.1715

Ugarte-Urra I, Doyle JG, Madjarska MS, O'Shea E (2004a) Signature of oscillations in coronal bright points. Astron Astrophys 418:313-324. https://doi.org/10.1051/0004-6361:20035666

Ugarte-Urra I, Doyle JG, Nakariakov VM, Foley CR (2004b) CDS wide slit time-series of EUV coronal bright points. Astron Astrophys 425:1083-1095. https://doi.org/10.1051/0004-6361:20041069

Ugarte-Urra I, Doyle JG, Del Zanna G (2005) Electron densities in EUV coronal bright points. Astron Astrophys 435:1169-1172. https://doi.org/10.1051/0004-6361:20052795

Ulyanov AS, Bogachev SA, Kuzin SV (2010) Bright points and ejections observed on the Sun by the KORONAS-FOTON instrument TESIS. Astron Rep 54:948-957. https://doi.org/10.1134/ S1063772910100082

Vaiana GS, Davis JM, Giacconi R, Krieger AS, Silk JK, Timothy AF, Zombeck M (1973a) X-ray observations of characteristic structures and time variations from the solar corona: preliminary results from Skylab. Astrophys J Lett 185:L47. https://doi.org/10.1086/181318

Vaiana GS, Krieger AS, Timothy AF (1973b) Identification and analysis of structures in the corona from X-ray photography. Sol Phys 32:81-116. https://doi.org/10.1007/BF00152731

Vaiana GS, Krieger AS, Timothy AF, Zombeck M (1976) ATM observations-X-ray results. Astrophys Space Sci 39:75-101. https://doi.org/10.1007/BF00640511

van Driel-Gesztelyi L, Green LM (2015) Evolution of active regions. Living Rev Sol Phys 12:1. https://doi. org/10.1007/lrsp-2015-1

Vilhu O, Huovelin J, Pohjolainen S, Virtanen J, Curdt W (2002) Microflaring of a solar bright point. Astron Astrophys 395:977-981. https://doi.org/10.1051/0004-6361:20021024. arXiv:astro-ph/0207274

von Rekowski B, Parnell CE, Priest ER (2006a) Solar coronal heating by magnetic cancellation-I. Connected equal bipoles. Mon Not R Astron Soc 366:125-136. https://doi.org/10.1111/j.1365-2966.2005. 09801.x

von Rekowski B, Parnell CE, Priest ER (2006b) Solar coronal heating by magnetic cancellation-II. Disconnected and unequal bipoles. Mon Not R Astron Soc 369:43-56. https://doi.org/10.1111/j.13652966.2006.10345.x 
Waldron WL, Mullan DJ (1987) Magnetohydrodynamic modeling of coronal bright points. Astrophys J 319:971-983. https://doi.org/10.1086/165514

Webb DF, Martin SF, Moses D, Harvey JW (1993) The correspondence between X-ray bright points and evolving magnetic features in the quiet Sun. Sol Phys 144:15-35. https://doi.org/10.1007/BF00667979

Wilhelm K, Lemaire P, Curdt W, Schuhle U, Marsch E, Poland AI, Jordan SD, Thomas RJ, Hassler DM, Huber MCE, Vial JC, Kuhne M, Siegmund OHW, Gabriel A, Timothy JG, Grewing M, Feldman U, Hollandt J, Brekke P (1997) First results of the SUMER telescope and spectrometer on SOHO-I. Spectra and spectroradiometry. Sol Phys 170:75-104. https://doi.org/10.1023/A:1004923511980

Woodgate BE, Tandberg-Hanssen EA, Bruner EC, Beckers JM, Brandt JC, Henze W, Hyder CL, Kalet MW, Kenny PJ, Knox ED, Michalitsianos AG, Rehse R, Shine RA, Tinsley HD (1980) The ultraviolet spectrometer and polarimeter on the Solar Maximum Mission. Sol Phys 65:73-90. https://doi.org/10. 1007/BF00151385

Wyper PF, DeVore CR, Karpen JT, Antiochos SK, Yeates AR (2018) A model for coronal hole bright points and jets due to moving magnetic elements. Astrophys J 864:165. https://doi.org/10.3847/1538-4357/ aad9f7. arXiv: 1808.03688

Xia LD, Marsch E, Curdt W (2003) On the outflow in an equatorial coronal hole. Astron Astrophys 399:L5L9. https://doi.org/10.1051/0004-6361:20030016

Yang S, Zhang J (2014) Properties of solar ephemeral regions at the emergence stage. Astrophys J 781:7. https://doi.org/10.1088/0004-637X/781/1/7. arXiv:1312.0334

Zhang J, Kundu MR, White SM (2001) Spatial distribution and temporal evolution of coronal bright points. Sol Phys 198:347-365. https://doi.org/10.1023/A:1005222616375

Zhang QM, Chen PF, Guo Y, Fang C, Ding MD (2012) Two types of magnetic reconnection in coronal bright points and the corresponding magnetic configuration. Astrophys J 746:19. https://doi.org/10. 1088/0004-637X/746/1/19

Zhang QM, Chen PF, Ding MD, Ji HS (2014) Reciprocatory magnetic reconnection in a coronal bright point. Astron Astrophys 568:A30. https://doi.org/10.1051/0004-6361/201322815. arXiv:1406.5328

Zirin H, Hurford GJ, Marsh KA (1978) The small-scale sources of quiet-Sun centimeter-wave radio emission. Astrophys J 224:1043-1047. https://doi.org/10.1086/156455

Publisher's Note Springer Nature remains neutral with regard to jurisdictional claims in published maps and institutional affiliations. 